\title{
LAWS OF RARE EVENTS FOR DETERMINISTIC AND RANDOM DYNAMICAL SYSTEMS
}

\author{
HALE AYTAÇ, JORGE MILHAZES FREITAS, AND SANDRO VAIENTI
}

Abstract. The object of this paper is twofold. From one side we study the dichotomy, in terms of the Extremal Index of the possible Extreme Value Laws, when the rare events are centred around periodic or non-periodic points. Then we build a general theory of Extreme Value Laws for randomly perturbed dynamical systems. We also address, in both situations, the convergence of Rare Events Point Processes. Decay of correlations against $L^{1}$ observables will play a central role in our investigations.

\section{Contents}

1. Introduction

2. Statement of results

3. Extremal Index dichotomy for deterministic systems

4. Extremes for random dynamics

5. Extremes for random dynamics from a spectral approach

Appendix A. Clustering and periodicity

Appendix B. Computing the EI and the multiplicity distribution

\section{INTRODUCTION}

Deterministic discrete dynamical systems are often used to model physical phenomena. In many situations, inevitable observation errors make it more realistic to

Received by the editors January 31, 2013 and, in revised form, September 13, 2013.

2010 Mathematics Subject Classification. Primary 37A50, 60G70, 37B20, 60G10, 37A25, $37 \mathrm{H} 99$.

Key words and phrases. Random dynamical systems, extreme values, hitting times statistics, extremal index.

The first author was partially supported by FCT (Portugal) grant SFRH/BD/33371/2008.

The first and second authors were supported by the European Regional Development Fund through the programme COMPETE and by the Portuguese Government through the FCT and CMUP under the project PEst-C/MAT/UI0144/2011.

The second author was partially supported by FCT grant SFRH/BPD/66040/2009 and by FCT project PTDC/MAT/099493/2008.

The third author was supported by the CNRS-PEPS Mathematical Methods of Climate Theory and by the ANR-Project Perturbations; part of this work was done while he was visiting the Centro de Modelamiento Matemático, UMI2807, in Santiago de Chile with a CNRS support (délégation).

All three authors were supported by FCT project PTDC/MAT/120346/2010, which is financed by national and European Community structural funds through the programs FEDER and COMPETE. 
consider random dynamics, where the mathematical model is adjusted by adding random noise to the iterative process in order to account for these practical imprecisions. The behaviour of such random systems has been studied thoroughly in the last decades. We mention, for example, 22,23] for excellent expositions on the subject.

Laws of rare events for chaotic (deterministic) dynamical systems have also been exhaustively studied in the last years. When these results first appeared these notions were described as Hitting Times Statistics (HTS) or Return Times Statistics (RTS). In this setting, rare events correspond to entrances in small regions of the phase space, and the goal is to prove distributional limiting laws for the normalised waiting times before hitting/returning to these asymptotically small sets. We refer to [29] for an excellent review. More recently, rare events have also been studied through Extreme Value Laws (EVLs), i.e., the distributional limit of the partial maxima of stochastic processes arising from such chaotic systems simply by evaluating an observable function along the orbits of the system. Very recently, in 10, 11, the two perspectives have been proved to be linked so that, under general conditions on the observable functions, the existence of HTS/RTS is equivalent to the existence of EVLs. These observable functions achieve a maximum (possibly $\infty)$ at some chosen point $\zeta$ in the phase space so that the rare event of occurring an exceedance of a high level corresponds to an entrance in a small ball around $\zeta$. The study of rare events may be enhanced if we enrich the process by considering multiple exceedances (or hits/returns to target sets) that are recorded by Rare Events Point Processes (REPP), which count the number of exceedances (or hits/returns) in a certain time frame. Then one looks for limits in distribution for such REPP when time is adequately normalised.

Surprisingly, not much is known about rare events for random dynamical systems. One of the main goals here is to establish what we believe to be the first result proving the existence of EVLs (or equivalently HTS/RTS) as well as the convergence of REPP, for randomly perturbed dynamical systems.

We remark that in the recent paper [26] the authors defined the meaning of first hitting/return time in the random dynamical setting. To our knowledge this was the first paper to address this issue of recurrence for random dynamics. There, the authors define the concepts of quenched and annealed return times for systems generated by the composition of random maps. Moreover, they prove that for super-polynomially mixing systems, the random recurrence rate is equal to the local dimension of the stationary measure.

Here we are interested in establishing the right setting in order to have the connection between EVL and HTS/RTS, for random dynamics, and, eventually, to prove the existence of EVLs and HTS/RTS for random orbits. Moreover, we also study the convergence of the REPP for randomly perturbed systems. These achievements are, in our opinion, the main accomplishments of this paper.

In general terms, we will consider uniformly expanding and piecewise expanding maps. Then we randomly perturb these discrete systems with additive, independent, identically distributed (i.i.d.) noise introduced at each iteration. The noise distribution is absolutely continuous with respect to (w.r.t.) Lebesgue measure. The details will be given in Section 2

The main ingredients will be decay of correlations against all $L^{1}$ observables (we mean decay of correlations of all observables in some Banach space against all 
observables in $L^{1}$, which will be made more precise in Definition 2.2 below) and the notion of first return time from a set to itself.

We realised that the techniques we were using to study the random scenario also allowed us to give an answer to one of the questions raised in [12. There the connection between periodicity, clustering of rare events and the Extremal Index (EI) was studied. In certain situations, like when rare events are defined as entrances in balls around (repelling) periodic points, the stochastic processes generated by the dynamics present clustering of rare events. The EI is a parameter $\vartheta \in[0,1]$ which quantifies the intensity of the clustering. In fact, in most situations the average cluster size is just $1 / \vartheta$. No clustering means that $\vartheta=1$ and strong clustering means that $\vartheta$ is close to 0 . In [12, Section 6], it is shown that, for uniformly expanding maps of the circle equipped with the Bernoulli measure, $f$ : $\mathcal{S}^{1} \rightarrow \mathcal{S}^{1}$ with $f(z)=z^{2}$, there is a dichotomy in terms of the possible EVL: either the rare events are centred at (repelling) periodic points and $\vartheta<1$ or at nonperiodic points and the EI is 1 . This was proved for cylinders, in the sense that rare events corresponded to entrances into dynamically defined cylinders (instead of balls), and one of the questions it raised was if this dichotomy could be proved more generally for balls and for more general systems. In [8], the authors build up on the work of [17] and eventually obtain the dichotomy for balls and for conformal repellers.

One of our results here, Theorem A, allows us to prove the dichotomy for balls and for systems with decay of correlations against $L^{1}$ which include, for example, piecewise expanding maps of the interval-like Rychlik maps (Proposition 3.2) or piecewise expanding maps in higher dimensions, like the ones studied by Saussol in [28. (Proposition 3.3). Moreover, as an end product of our approach, we can express the dichotomy for these systems in the following more general terms (see Propositions 3.2 and 3.3): either we have, at non-periodic points, the convergence of the REPP to the standard Poisson process or we have, at repelling periodic points, the convergence of REPP to a compound Poisson process consisting of an underlying asymptotic Poisson process governing the positions of the clusters of exceedances and a multiplicity distribution associated to each such Poisson event, which is determined by the average cluster size. In fact, at repelling periodic points, we always get that the multiplicity distribution is the geometric distribution (see [13, 16]).

We also consider discontinuity points of the map as centres of the rare events (see Proposition 3.4). A very interesting immediate consequence of this study is that, when we consider the REPP, we can obtain convergence to a compound Poisson process whose multiplicity distribution is not a geometric distribution. To our knowledge this is the first time these limits are obtained for the general piecewise expanding systems considered and in the balls' setting (rather than cylinders), in the sense that exceedances or rare events correspond to the entrance of the orbits in topological balls.

In the course of writing this paper we came across a paper by Keller, 20, where he proved the dichotomy of expanding maps with a spectral gap for the corresponding Perron-Frobenius operator (which also include Rychlik maps and the higherdimensional piecewise expanding maps studied by Saussol [28], for example). He uses of a powerful technique developed in [21, based on an eigenvalue perturbation formula. Our approach here is different since we use an EVL kind of argument and 
our assumptions are based on decay of correlations against $L^{1}$ observables. Moreover, in this paper we also deal with the convergence of the REPP and obtain, in particular, the interesting fact that at discontinuity points we observe multiplicity distributions other than the geometric one.

We also mention the very recent paper [24], where the dichotomy for cylinders is established for mixing countable alphabet shifts, but also in the context of nonconventional ergodic sums. It also includes examples of non-convergence of the $\mathrm{REPP}$, in the cylinder setting.

We remark that in most situations, decay of correlations against $L^{1}$ observables is a consequence of the existence of a gap in the spectrum of the map's corresponding Perron-Frobenius operator. However, in [7], Dolgopyat proves exponential decay of correlations for certain Axiom A flows, but along the way he proves it for semiflows against $L^{1}$ observables. This is done via estimates on families of twisted transfer operators for the Poincaré map, but without considering the Perron-Frobenius operator for the flow itself. This means that the discretisation of this flow by using a time 1 map, for example, provides an example of a system with decay of correlations against $L^{1}$ for which it is not known if there exists a spectral gap of the corresponding Perron-Frobenius operator. Apparently, the existence of a spectral gap for the map's Perron-Frobenius operator, defined in some nice function space, implies decay of correlations against $L^{1}$ observables. However, the latter is still a very strong property. In fact, from decay of correlations against $L^{1}$ observables, regardless of the rate, as long as it is summable, one can actually show that the system has exponential decay of correlations of Hölder observables against $L^{\infty}$. (See [1. Theorem B].) So an interesting question is:

Question. If a system presents summable decay of correlations against $L^{1}$ observables, is there a spectral gap for the system's Perron-Frobenius operator, defined in some appropriate function space?

We note that, as we point out in Remark 3.1 we do not actually need decay of correlations against $L^{1}$ in its full strength.

Returning to the random setting, our main result asserts that the dichotomy observed for deterministic systems vanishes, and regardless of the centre being a periodic point or not, we always get standard exponential EVLs or, equivalently, standard exponential HTS/RTS (which means that $\vartheta=1$ ). Moreover, we also show that the REPP converges in distribution to a standard Poisson process. We will prove these results in Section 4 using an EVL approach, where the main assumption will be decay of correlations against $L^{1}$.

Still in the random setting, motivated by the deep work of Keller, 20], in Section 5 we prove results in the same directions as before but based on the spectral approach used by Keller and Liverani to study deterministic systems. As a byproduct we get an HTS/RTS formula with sharp error terms for random dynamical systems (see Proposition 5.1). We will point out the differences between the two techniques (which we name here as direct and spectral, respectively) at the beginning of Section 5; let us simply stress that we implemented the spectral technique in a random situation only for one-dimensional systems and the existence of EI was proved for a substantially large class of noises. On the other hand, the direct technique worked for systems in higher dimensions as well, but it required additive noise with a continuous distribution. However, the latter was necessary to prove that EI is 1 in the spectral approach too. 


\section{Statement of Results}

Consider a discrete time dynamical system $(\mathcal{X}, \mathcal{B}, \mathbb{P}, T)$ which will denote two different but interrelated settings throughout the paper. $\mathcal{X}$ is a topological space, $\mathcal{B}$ is the Borel $\sigma$-algebra, $T: \mathcal{X} \rightarrow \mathcal{X}$ is a measurable map and $\mathbb{P}$ is a $T$-invariant probability measure, i.e., $\mathbb{P}\left(T^{-1}(B)\right)=\mathbb{P}(B)$, for all $B \in \mathcal{B}$. Also, given any $A \in \mathcal{B}$ with $\mathbb{P}(A)>0$, let $\mathbb{P}_{A}$ denote the conditional measure on $A \in \mathcal{B}$, i.e., $\mathbb{P}_{A}:=\frac{\left.\mathbb{P}\right|_{A}}{\mathbb{P}(A)}$.

Firstly, it will denote a deterministic setting where $\mathcal{X}=\mathcal{M}$ is a compact Riemannian manifold, $\mathcal{B}$ is the Borel $\sigma$-algebra, $T=f: \mathcal{M} \rightarrow \mathcal{M}$ is a piecewise differentiable map and $\mathbb{P}=\mu$ is an $f$-invariant probability measure. Let $\operatorname{dist}(\cdot, \cdot)$ denote a Riemannian metric on $\mathcal{M}$ and Leb a normalised volume form on the Borel sets of $\mathcal{M}$ that we call Lebesgue measure.

Secondly, it will denote a random setting which is constructed from the deterministic system via perturbing the original map with random additive noise. We assume that $\mathcal{M}$ is a quotient of a Banach vector space $\mathcal{V}$, like $\mathcal{M}=\mathbb{T}^{d}=\mathbb{R}^{d} / \mathbb{Z}^{d}$, for some $d \in \mathbb{N}$. In the case $d=1$, we will also denote the circle $\mathbb{T}^{1}$ by $\mathcal{S}^{1}$. Let $\operatorname{dist}(\cdot, \cdot)$ denote the induced usual quotient metric on $\mathcal{M}$ and Leb a normalised volume form on the Borel sets of $\mathcal{M}$ that we call Lebesgue measure. Also denote the ball of radius $\varepsilon>0$ around $x \in \mathcal{M}$ by $B_{\varepsilon}(x):=\{y \in \mathcal{M}: \operatorname{dist}(x, y)<\varepsilon\}$. Consider the unperturbed deterministic system $f: \mathcal{M} \rightarrow \mathcal{M}$. For some $\varepsilon>0$, let $\theta_{\varepsilon}$ be a probability measure defined on the Borel subsets of $B_{\varepsilon}(0)$, such that

$$
\theta_{\varepsilon}=g_{\varepsilon} \text { Leb } \quad \text { and } \quad 0<\underline{g_{\varepsilon}} \leq g_{\varepsilon} \leq \overline{g_{\varepsilon}}<\infty .
$$

For each $\omega \in B_{\varepsilon}(0)$, we define the additive perturbation of $f$ that we denote by $f_{\omega}$ as the map $f_{\omega}: \mathcal{M} \rightarrow \mathcal{M}$, given by

$$
f_{\omega}(x)=f(x)+\omega .
$$

Consider a sequence of i.i.d. random variables (r.v.) $W_{1}, W_{2}, \ldots$ taking values on $B_{\varepsilon}(0)$ with common distribution given by $\theta_{\varepsilon}$. Let $\Omega=B_{\varepsilon}(0)^{\mathbb{N}}$ denote the space of realisations of such a process and $\theta_{\varepsilon}^{\mathbb{N}}$ the product measure defined on its Borel subsets. Given a point $x \in \mathcal{M}$ and the realisation of the stochastic process $\underline{\omega}=$ $\left(\omega_{1}, \omega_{2}, \ldots\right) \in \Omega$, we define the random orbit of $x$ as $x, f_{\underline{\omega}}(x), f_{\underline{\omega}}^{2}(x), \ldots$, where the evolution of $x$, up to time $n \in \mathbb{N}$, is obtained by the concatenation of the respective additive randomly perturbed maps in the following way:

$$
f_{\underline{\omega}}^{n}(x)=f_{\omega_{n}} \circ f_{\omega_{n-1}} \circ \cdots \circ f_{\omega_{1}}(x),
$$

with $f_{\omega}^{0}$ being the identity map on $\mathcal{M}$. The next definition gives a notion that plays the role of invariance in the deterministic setting.

\footnotetext{
${ }^{1}$ In the general theory of randomly perturbed dynamical systems one could consider perturbations other than the additive ones and distributions $\theta_{\varepsilon}$ which are not necessarily absolutely continuous. Our choice is motivated by the fact that our main result for the extreme values in presence of noise could be relatively easily shown with those assumptions, but it is also clear from the proof where possible generalizations could occur. We were especially concerned with constructing the framework and finding good assumptions for the theory, which is surely satisfied for more general perturbations and probability distributions. Let us notice that other authors basically used additive noise when they studied statistical properties of random dynamical systems [2], for instance.
} 
Definition 2.1. Given $\varepsilon>0$, we say that the probability measure $\mu_{\varepsilon}$ on the Borel subsets of $\mathcal{M}$ is stationary if

$$
\iint \phi\left(f_{\omega}(x)\right) \mathrm{d} \mu_{\varepsilon}(x) \mathrm{d} \theta_{\varepsilon}(\omega)=\int \phi(x) \mathrm{d} \mu_{\varepsilon}(x),
$$

for every $\phi: \mathcal{M} \rightarrow \mathbb{R}$ integrable w.r.t. $\mu_{\varepsilon}$.

The previous equality could also be written as

$$
\int \mathcal{U}_{\varepsilon} \phi \mathrm{d} \mu_{\varepsilon}=\int \phi \mathrm{d} \mu_{\varepsilon}
$$

where the operator $\mathcal{U}_{\varepsilon}: L^{\infty}($ Leb $) \rightarrow L^{\infty}($ Leb $)$, is defined as

$$
\left(\mathcal{U}_{\varepsilon} \phi\right)(x)=\int_{B_{\varepsilon}(0)} \phi\left(f_{\omega}(x)\right) \mathrm{d} \theta_{\varepsilon}
$$

and is called the random evolution operator.

The adjoint of this operator is called the random Perron-Frobenius operator, $\mathcal{P}_{\varepsilon}: L^{1}($ Leb $) \rightarrow L^{1}($ Leb), and it acts by duality as

$$
\int \mathcal{P}_{\varepsilon} \psi \cdot \phi \mathrm{dLeb}=\int \mathcal{U}_{\varepsilon} \phi \cdot \psi \mathrm{dLeb},
$$

where $\psi \in L^{1}$ and $\phi \in L^{\infty}$.

It is immediate from this definition to get another useful representation of this operator, namely for $\psi \in L^{1}$ :

$$
\left(\mathcal{P}_{\varepsilon} \psi\right)(x)=\int_{B_{\varepsilon}(0)}\left(\mathcal{P}_{\omega} \psi\right)(x) \mathrm{d} \theta_{\varepsilon}(\omega),
$$

where $\mathcal{P}_{\omega}$ is the Perron-Frobenius operator associated to $f_{\omega}$.

We recall that the stationary measure $\mu_{\varepsilon}$ is absolutely continuous w.r.t. the Lebesgue measure and with density $h_{\varepsilon}$ if and only if such a density is a fixed point of the random Perron-Frobenius operator: $\mathcal{P}_{\varepsilon} h_{\varepsilon}=h_{\varepsilon}$ 2

We can give a deterministic representation of this random setting using the following skew product transformation:

$$
\begin{aligned}
S: \mathcal{M} \times \Omega & \longrightarrow \mathcal{M} \times \Omega, \\
(x, \underline{\omega}) & \longmapsto\left(f_{\omega_{1}}, \sigma(\underline{\omega})\right),
\end{aligned}
$$

where $\sigma: \Omega \rightarrow \Omega$ is the one-sided shift $\sigma(\underline{\omega})=\sigma\left(\omega_{1}, \omega_{2}, \ldots\right)=\left(\omega_{2}, \omega_{3}, \ldots\right)$. We remark that $\mu_{\varepsilon}$ is stationary if and only if the product measure $\mu_{\varepsilon} \times \theta_{\varepsilon}^{\mathbb{N}}$ is an $S$-invariant measure.

Hence, the random evolution can fit the original model $(\mathcal{X}, \mathcal{B}, \mathbb{P}, T)$ by taking the product space $\mathcal{X}=\mathcal{M} \times \Omega$, with the corresponding product Borel $\sigma$-algebra $\mathcal{B}$, where the product measure $\mathbb{P}=\mu_{\varepsilon} \times \theta_{\epsilon}^{\mathbb{N}}$ is defined. The system is then given by the skew product map $T=S$.

For the systems we will consider, $\mathbb{P}$ has very good mixing properties, which in loose terms means that the system loses memory quite fast. In order to quantify the memory loss we look at the system's rates of decay of correlations w.r.t. $\mathbb{P}$.

\footnotetext{
${ }^{2}$ The duality explains why we take $\mathcal{P}_{\varepsilon}$ acting on $L^{1}$ and $\mathcal{U}_{\varepsilon}$ on $L^{\infty}$. Moreover, our stationary measures will be absolutely continuous with density given by the fixed point of the PerronFrobenius operator $\mathcal{P}_{\varepsilon}$.
} 
Definition 2.2 (Decay of correlations). Let $\mathcal{C}_{1}, \mathcal{C}_{2}$ denote Banach spaces of real valued measurable functions defined on $\mathcal{X}$. We denote the correlation of non-zero functions $\phi \in \mathcal{C}_{1}$ and $\psi \in \mathcal{C}_{2}$ w.r.t. a measure $\mathbb{P}$ as

$$
\operatorname{Cor}_{\mathbb{P}}(\phi, \psi, n):=\frac{1}{\|\phi\|_{\mathcal{C}_{1}}\|\psi\|_{\mathcal{C}_{2}}}\left|\int \phi\left(\psi \circ T^{n}\right) \mathrm{d} \mathbb{P}-\int \phi \mathrm{d} \mathbb{P} \int \psi \mathrm{d} \mathbb{P}\right| .
$$

We say that we have decay of correlations, w.r.t. the measure $\mathbb{P}$, for observables in $\mathcal{C}_{1}$ against observables in $\mathcal{C}_{2}$ if, for every $\phi \in \mathcal{C}_{1}$ and every $\psi \in \mathcal{C}_{2}$, we have

$$
\operatorname{Cor}_{\mathbb{P}}(\phi, \psi, n) \rightarrow 0, \quad \text { as } n \rightarrow \infty .
$$

In the random setting, we will only be interested in Banach spaces of functions that do not depend on $\underline{\omega} \in \Omega$. Hence, we assume that $\phi, \psi$ are actually functions defined on $\mathcal{M}$ and the correlation between these two observables can be written more simply as

$$
\begin{aligned}
\operatorname{Cor}_{\mathbb{P}}(\phi, \psi, n): & =\frac{1}{\|\phi\|_{\mathcal{C}_{1}}\|\psi\|_{\mathcal{C}_{2}}}\left|\int\left(\int \psi \circ f_{\underline{\omega}}^{n} \mathrm{~d} \theta_{\varepsilon}^{\mathbb{N}}\right) \phi \mathrm{d} \mu_{\varepsilon}-\int \phi \mathrm{d} \mu_{\varepsilon} \int \psi \mathrm{d} \mu_{\varepsilon}\right| \\
& =\frac{1}{\|\phi\|_{\mathcal{C}_{1}}\|\psi\|_{\mathcal{C}_{2}}}\left|\int \mathcal{U}_{\varepsilon}^{n} \psi \cdot \phi \mathrm{d} \mu_{\varepsilon}-\int \phi \mathrm{d} \mu_{\varepsilon} \int \psi \mathrm{d} \mu_{\varepsilon}\right|,
\end{aligned}
$$

where $\left(\mathcal{U}_{\varepsilon}^{n} \psi\right)(x)=\int \cdots \int \psi\left(f_{\omega_{n}} \circ \cdots \circ f_{\omega_{1}} x\right) \mathrm{d} \theta_{\varepsilon}\left(\omega_{n}\right) \ldots \mathrm{d} \theta_{\varepsilon}\left(\omega_{1}\right)=\int \psi \circ f_{\underline{\omega}}^{n}(x) \mathrm{d} \theta_{\varepsilon}^{\mathbb{N}}$.

We say that we have decay of correlations against $L^{1}$ observables whenever we have decay of correlations, with respect to the measure $\mathbb{P}$, for observables in $\mathcal{C}_{1}$ against observables in $\mathcal{C}_{2}$ and $\mathcal{C}_{2}=L^{1}$ (Leb) is the space of Leb-integrable functions on $\mathcal{M}$ and $\|\psi\|_{\mathcal{C}_{2}}=\|\psi\|_{1}=\int|\psi|$ dLeb. Note that when $\mu, \mu_{\varepsilon}$ are absolutely continuous with respect to Leb and the respective Radon-Nikodym derivatives are bounded above and below by positive constants, then $L^{1}(\mathrm{Leb})=L^{1}(\mu)=L^{1}\left(\mu_{\varepsilon}\right)$.

The goal is to study the statistical properties of such systems regarding the occurrence of rare events. There are two approaches for this purpose which were recently proved to be equivalent.

We first turn to the existence of an EVL for the partial maximum of observations made along the time evolution of the system. To be more precise consider the time series $X_{0}, X_{1}, X_{2}, \ldots$ arising from such a system simply by evaluating a given random variable (r.v.) $\varphi: \mathcal{M} \rightarrow \mathbb{R} \cup\{+\infty\}$ along the orbits of the system:

$$
X_{n}=\varphi \circ f^{n}, \quad \text { for each } n \in \mathbb{N} \text {. }
$$

Note that when we consider the random dynamics, the process will be

$$
X_{n}=\varphi \circ f_{\underline{\omega}}^{n}, \quad \text { for each } n \in \mathbb{N},
$$

which can also be written as $X_{n}=\bar{\varphi} \circ S^{n}$, where

$$
\begin{array}{ccc}
\bar{\varphi}: \mathcal{M} \times \Omega & \longrightarrow \mathbb{R} \cup\{+\infty\}, \\
(x, \underline{\omega}) & \longmapsto & \varphi(x) .
\end{array}
$$

Clearly, $X_{0}, X_{1}, \ldots$ defined in this way is not an independent sequence. However, invariance of $\mu$ and stationarity of $\mu_{\varepsilon}$ guarantee that the stochastic process is stationary in both cases.

We assume that the r.v. $\varphi: \mathcal{M} \rightarrow \mathbb{R} \cup\{ \pm \infty\}$ achieves a global maximum at $\zeta \in \mathcal{M}$ (we allow $\varphi(\zeta)=+\infty$ ). We also assume that $\varphi$ and $\mathbb{P}$ are sufficiently 
regular so that:

(R1) for $u$ sufficiently close to $u_{F}:=\varphi(\zeta)$, the event

$$
U(u)=\left\{X_{0}>u\right\}=\{x \in \mathcal{M}: \varphi(x)>u\}
$$

corresponds to a topological ball centred at $\zeta$. Moreover, the quantity $\mathbb{P}(U(u))$, as a function of $u$, varies continuously on a neighbourhood of $u_{F}$.

In what follows, an exceedance of the level $u \in \mathbb{R}$ at time $j \in \mathbb{N}$ means that the event $\left\{X_{j}>u\right\}$ occurs. We denote by $F$ the distribution function (d.f.) of $X_{0}$, i.e., $F(x)=\mathbb{P}\left(X_{0} \leq x\right)$. Given any d.f. $G$, let $\bar{G}=1-G$ and $u_{G}$ denote the right endpoint of the d.f. $G$, i.e., $u_{G}=\sup \{x: G(x)<1\}$.

The idea then is to consider the extremal behaviour of the system for which we define a new sequence of random variables $M_{1}, M_{2}, \ldots$ given by

$$
M_{n}=\max \left\{X_{0}, \ldots, X_{n-1}\right\} .
$$

Definition 2.3. We say that we have an $E V L$ for $M_{n}$ if there is a non-degenerate d.f. $H: \mathbb{R} \rightarrow[0,1]$ with $H(0)=0$ and, for every $\tau>0$, there exists a sequence of levels $u_{n}=u_{n}(\tau), n=1,2, \ldots$, such that

$$
n \mathbb{P}\left(X_{0}>u_{n}\right) \rightarrow \tau, \text { as } n \rightarrow \infty,
$$

and for which the following holds:

$$
\mathbb{P}\left(M_{n} \leq u_{n}\right) \rightarrow \bar{H}(\tau), \text { as } n \rightarrow \infty .
$$

Remark 2.1. We remark that one of the advantages of the EVL approach for the study of rare events for random dynamics is that its definition follows straightforwardly from the deterministic case. In fact, the only difference is that for random dynamical systems, the r.v. $M_{n}$ 's are defined on $\mathcal{M} \times \Omega$ where we use the measure $\mathbb{P}=\mu_{\varepsilon} \times \theta_{\varepsilon}^{\mathbb{N}}$ as opposed to the deterministic case where the ambient space is $\mathcal{M}$ and $\mathbb{P}=\mu$.

The motivation for using a normalising sequence $u_{n}$ satisfying (2.10) comes from the case when $X_{0}, X_{1}, \ldots$ are independent and identically distributed. In this i.i.d. setting, it is clear that $\mathbb{P}\left(M_{n} \leq u\right)=(F(u))^{n}$. Hence, condition (2.10) implies that

$$
\mathbb{P}\left(M_{n} \leq u_{n}\right)=\left(1-\mathbb{P}\left(X_{0}>u_{n}\right)\right)^{n} \sim\left(1-\frac{\tau}{n}\right)^{n} \rightarrow \mathrm{e}^{-\tau},
$$

as $n \rightarrow \infty$. Moreover, the reciprocal is also true. Note that in this case $H(\tau)=$ $1-\mathrm{e}^{-\tau}$ is the standard exponential d.f.

For every sequence $\left(u_{n}\right)_{n \in \mathbb{N}}$ satisfying (2.10) we define:

$$
U_{n}:=\left\{X_{0}>u_{n}\right\} \text {. }
$$

When $X_{0}, X_{1}, X_{2}, \ldots$ are not independent, the standard exponential law still applies under some conditions on the dependence structure. These conditions are the following:

Condition $\left(D_{2}\left(u_{n}\right)\right)$. We say that $D_{2}\left(u_{n}\right)$ holds for the sequence $X_{0}, X_{1}, \ldots$ if for all $\ell, t$ and $n$,

$$
\left|\mathbb{P}\left(X_{0}>u_{n} \cap \max \left\{X_{t}, \ldots, X_{t+\ell-1} \leq u_{n}\right\}\right)-\mathbb{P}\left(X_{0}>u_{n}\right) \mathbb{P}\left(M_{\ell} \leq u_{n}\right)\right| \leq \gamma(n, t),
$$

where $\gamma(n, t)$ is decreasing in $t$ for each $n$ and $n \gamma\left(n, t_{n}\right) \rightarrow 0$ when $n \rightarrow \infty$ for some sequence $t_{n}=o(n)$. 
Now, let $\left(k_{n}\right)_{n \in \mathbb{N}}$ be a sequence of integers such that

$$
k_{n} \rightarrow \infty \text { and } k_{n} t_{n}=o(n) .
$$

Condition $\left(D^{\prime}\left(u_{n}\right)\right)$. We say that $D^{\prime}\left(u_{n}\right)$ holds for the sequence $X_{0}, X_{1}, X_{2}, \ldots$ if there exists a sequence $\left(k_{n}\right)_{n \in \mathbb{N}}$ satisfying (2.13) and such that

$$
\lim _{n \rightarrow \infty} n \sum_{j=1}^{\left\lfloor n / k_{n}\right\rfloor} \mathbb{P}\left(X_{0}>u_{n}, X_{j}>u_{n}\right)=0 .
$$

By [9. Theorem 1], if conditions $D_{2}\left(u_{n}\right)$ and $D^{\prime}\left(u_{n}\right)$ hold for $X_{0}, X_{1}, \ldots$, then there exists an EVL for $M_{n}$ and $H(\tau)=1-\mathrm{e}^{-\tau}$. Besides, as can be seen in [9, Section 2], condition $D_{2}\left(u_{n}\right)$ follows immediately if $X_{0}, X_{1}, \ldots$ is given by (2.6) and the system has a sufficiently fast decay of correlations.

Now, we turn to the other approach which regards the existence of HTS and RTS. In the deterministic case, consider a set $A \in \mathcal{B}$. We define a function that we refer to as first hitting time function to $A$ and denote it by $r_{A}: \mathcal{X} \rightarrow \mathbb{N} \cup\{+\infty\}$ where

$$
r_{A}(x)=\min \left\{j \in \mathbb{N} \cup\{+\infty\}: f^{j}(x) \in A\right\} .
$$

The restriction of $r_{A}$ to $A$ is called the first return time function to $A$. We define the first return time to $A$, which we denote by $R(A)$, as the minimum of the return time function to $A$, i.e.,

$$
R(A)=\min \left\{r_{A}(x): x \in A\right\} .
$$

In the random case, we have to make a choice regarding the type of definition we want to play the roles of the first hitting/return times (functions). Essentially, there are two possibilities. The first is the quenched perspective, which consists of fixing a realisation $\underline{\omega} \in \Omega$ and defining the objects in the same way as in the deterministic case. The second is the annealed perspective, which consists of defining the same objects by averaging over all possible realisations $\underline{\omega}$. Here, we will use the quenched perspective to define hitting/return times because it will facilitate the connection between EVLs and HTS/RTS in the random setting. (We refer to [26] for more details on both perspectives.)

For some $\underline{\omega} \in \Omega$ fixed, some $x \in \mathcal{M}$ and $A \subset \mathcal{M}$ measurable, we may define the first random hitting time

$$
r \frac{\omega}{A}(x):=\min \left\{j \in \mathbb{N}: f_{\underline{\omega}}^{j}(x) \in A\right\}
$$

and the first random return from $A$ to $A$ as

$$
R^{\underline{\omega}}(A)=\min \left\{r \frac{\omega}{A}(x): x \in A\right\} .
$$

Definition 2.4. Given a sequence of measurable subsets of $\mathcal{X},\left(V_{n}\right)_{n \in \mathbb{N}}$, so that $\mathbb{P}\left(V_{n}\right) \rightarrow 0$, the system has (random) HTS $G$ for $\left(V_{n}\right)_{n \in \mathbb{N}}$ if for all $t \geq 0$

$$
\mathbb{P}\left(r_{V_{n}} \leq \frac{t}{\mathbb{P}\left(V_{n}\right)}\right) \rightarrow G(t) \text { as } n \rightarrow \infty,
$$

and the system has (random) RTS $\tilde{G}$ for $\left(V_{n}\right)_{n \in \mathbb{N}}$ if for all $t \geq 0$

$$
\mathbb{P}_{V_{n}}\left(r_{V_{n}} \leq \frac{t}{\mathbb{P}\left(V_{n}\right)}\right) \rightarrow \tilde{G}(t) \text { as } n \rightarrow \infty \text {. }
$$


In the deterministic case, $\mathcal{X}=\mathcal{M}, \mathbb{P}=\mu$ and $T=f$. In the random case, $\mathcal{X}=\mathcal{M} \times \Omega, \mathbb{P}=\mu_{\varepsilon} \times \theta_{\varepsilon}^{\mathbb{N}}, T=S$ defined in (2.4),$V_{n}=V_{n}^{*} \times \Omega$, where $V_{n}^{*} \subset \mathcal{M}$ and $\mu_{\varepsilon}\left(V_{n}^{*}\right) \rightarrow 0$, as $n \rightarrow \infty$.

Note that

$$
\mathbb{P}\left(r_{V_{n}} \leq \frac{t}{\mathbb{P}\left(V_{n}\right)}\right)=\mu_{\varepsilon} \times \theta_{\varepsilon}^{\mathbb{N}}\left(r \frac{\omega}{V_{n}^{*}} \leq \frac{t}{\mu_{\varepsilon}\left(V_{n}^{*}\right)}\right)
$$

The normalising sequences to obtain HTS/RTS are motivated by Kac's Lemma, which states that the expected value of $r_{A}$ w.r.t. $\mu_{A}$ is $\int_{A} r_{A} d \mu_{A}=1 / \mu(A)$. So in studying the fluctuations of $r_{A}$ on $A$, the relevant normalising factor should be $1 / \mu(A)$.

The existence of exponential HTS is equivalent to the existence of exponential RTS. In fact, according to the Main Theorem in [15], a system has HTS $G$ if and only if it has RTS $\tilde{G}$ and

$$
G(t)=\int_{0}^{t}(1-\tilde{G}(s)) \mathrm{d} s .
$$

In [10, the link between HTS/RTS (for balls) and EVLs of stochastic processes given by (2.6) was established for invariant measures $\mu$ absolutely continuous w.r.t. Leb. Essentially, it was proved that if such a time series has an EVL $H$, then the system has HTS $H$ for balls "centred" at $\zeta$ and vice versa. (Recall that having HTS $H$ is equivalent to saying that the system has RTS $\tilde{H}$, where $H$ and $\tilde{H}$ are related by (2.17).) This was based on the elementary observation that for stochastic processes given by (2.6) we have

$$
f^{-1}\left(\left\{M_{n} \leq u\right\}\right)=\left\{r_{\left\{X_{0}>u\right\}}>n\right\} .
$$

This connection was exploited to prove EVLs using tools from HTS/RTS and the other way around. In [11, we carried the connection further to include more general measures, which, in particular, allows us to obtain the connection in the random setting. To check this we just need to use the skew product map to look at the random setting as a deterministic system and take the observable $\bar{\varphi}: \mathcal{M} \times \Omega \rightarrow$ $\mathbb{R} \cup\{+\infty\}$ defined as in (2.8) with $\varphi: \mathcal{M} \rightarrow \mathbb{R} \cup\{+\infty\}$ as in [11, equation (4.1)]. Then Theorems 1 and 2 from [1] guarantee that if we have an EVL, in the sense that (2.11) holds for some d.f. $H$, then we have HTS for sequences $\left\{V_{n}\right\}_{n \in \mathbb{N}}$, where $V_{n}=B_{\delta_{n}} \times \Omega$ and $\delta_{n} \rightarrow 0$ as $n \rightarrow \infty$, with $G=H$ and vice versa.

\section{Lemma 2.1 .}

$$
S^{-1}\left(\left\{M_{n} \leq u_{n}\right\}\right)=\left\{(x, \underline{\omega}) \in \mathcal{M} \times \Omega: r \frac{\omega}{U_{n}}(x)>n\right\} .
$$

Proof.

$$
\begin{aligned}
S^{-1}\left(\left\{M_{n} \leq u_{n}\right\}\right) & =S^{-1}\left(\left\{(x, \underline{\omega}) \in \mathcal{M} \times \Omega: x \notin U_{n}, r_{U_{n}}^{\omega}(x) \geq n\right\}\right)=: S^{-1}(A) \\
& =\left\{(x, \underline{\omega}) \in \mathcal{M} \times \Omega: S(x, \underline{\omega})=\left(f_{\omega_{1}}(x), \sigma(\underline{\omega})\right) \in A\right\} \\
& =\left\{(x, \underline{\omega}) \in \mathcal{M} \times \Omega: f_{\omega_{1}}(x) \notin U_{n}, r_{U_{n}}^{\sigma(\underline{\omega})}\left(f_{\omega_{1}}(x)\right) \geq n\right\} \\
& =\left\{(x, \underline{\omega}) \in \mathcal{M} \times \Omega: r r_{U_{n}}(x)>n\right\} .
\end{aligned}
$$


If we consider multiple exceedances we are led to point processes of rare events counting the number of exceedances in a certain time frame. For every $A \subset \mathbb{R}$ we define

$$
\mathscr{N}_{u}(A):=\sum_{i \in A \cap \mathbb{N}_{0}} \mathbf{1}_{X_{i}>u}
$$

In the particular case where $A=I=[a, b)$ we simply write $\mathscr{N}_{u, a}^{b}:=\mathscr{N}_{u}([a, b))$. Observe that $\mathscr{N}_{u, 0}^{n}$ counts the number of exceedances amongst the first $n$ observations of the process $X_{0}, X_{1}, \ldots, X_{n}$ or, in other words, the number of entrances in $U(u)$ up to time $n$. Also, note that

$$
\left\{\mathscr{N}_{u, 0}^{n}=0\right\}=\left\{M_{n} \leq u\right\} .
$$

In order to define a point process that captures the essence of an EVL and HTS through (2.19), we need to re-scale time using the factor $v:=1 / \mathbb{P}(X>u)$ given by Kac's Theorem. However, before we give the definition, we need some formalism. Let $\mathcal{S}$ denote the semi-ring of subsets of $\mathbb{R}_{0}^{+}$whose elements are intervals of the type $[a, b)$, for $a, b \in \mathbb{R}_{0}^{+}$. Let $\mathcal{R}$ denote the ring generated by $\mathcal{S}$. Recall that for every $J \in \mathcal{R}$ there are $k \in \mathbb{N}$ and $k$ intervals $I_{1}, \ldots, I_{k} \in \mathcal{S}$ such that $J=\bigcup_{i=1}^{k} I_{j}$. In order to fix notation, let $a_{j}, b_{j} \in \mathbb{R}_{0}^{+}$be such that $I_{j}=\left[a_{j}, b_{j}\right) \in \mathcal{S}$. For $I=[a, b) \in \mathcal{S}$ and $\alpha \in \mathbb{R}$, we denote $\alpha I:=[\alpha a, \alpha b)$ and $I+\alpha:=[a+\alpha, b+\alpha)$. Similarly, for $J \in \mathcal{R}$ define $\alpha J:=\alpha I_{1} \cup \cdots \cup \alpha I_{k}$ and $J+\alpha:=\left(I_{1}+\alpha\right) \cup \cdots \cup\left(I_{k}+\alpha\right)$.

Definition 2.5. We define the Rare Event Point Process (REPP) by counting the number of exceedances (or hits to $U\left(u_{n}\right)$ ) during the (re-scaled) time period $v_{n} J \in \mathcal{R}$, where $J \in \mathcal{R}$. To be more precise, for every $J \in \mathcal{R}$, set

$$
N_{n}(J):=\mathscr{N}_{u_{n}}\left(v_{n} J\right)=\sum_{j \in v_{n} J \cap \mathbb{N}_{0}} \mathbf{1}_{X_{j}>u_{n}} .
$$

Under dependence conditions similar to the ones just seen above, the REPP just defined converges in distribution to a standard Poisson process, when no clustering is involved, and to a compound Poisson process with intensity $\theta$ and a geometric multiplicity d.f., otherwise. For completeness, here we define what we mean by a Poisson and a compound Poisson process. (See [18] for more details.)

Definition 2.6. Let $T_{1}, T_{2}, \ldots$ be an i.i.d. sequence of random variables with common exponential distribution of mean $1 / \theta$. Let $D_{1}, D_{2}, \ldots$ be another i.i.d. sequence of random variables, independent of the previous one, and with d.f. $\pi$. Given these sequences, for $J \in \mathcal{R}$, set

$$
N(J)=\int \mathbf{1}_{J} d\left(\sum_{i=1}^{\infty} D_{i} \delta_{T_{1}+\ldots+T_{i}}\right),
$$

where $\delta_{t}$ denotes the Dirac measure at $t>0$. Whenever we are in this setting, we say that $N$ is a compound Poisson process of intensity $\theta$ and multiplicity d.f. $\pi$.

Remark 2.2. In this paper, the multiplicity will always be integer valued, which means that $\pi$ is completely defined by the values $\pi_{k}=\mathbb{P}\left(D_{1}=k\right)$, for every $k \in \mathbb{N}_{0}$. Note that, if $\pi_{1}=1$ and $\theta=1$, then $N$ is the standard Poisson process and, for every $t>0$, the random variable $N([0, t))$ has a Poisson distribution of mean $t$. 
Remark 2.3. When clustering is involved, we will see that $\pi$ is actually a geometric distribution of parameter $\theta \in(0,1)$, i.e., $\pi_{k}=\theta(1-\theta)^{k}$, for every $k \in \mathbb{N}_{0}$. This means that, as in [16], here the random variable $N([0, t))$ follows a Pólya-Aeppli distribution, i.e.:

$$
\mathbb{P}(N([0, t))=k)=\mathrm{e}^{-\theta t} \sum_{j=1}^{k} \theta^{j}(1-\theta)^{k-j} \frac{(\theta t)^{j}}{j !}\left(\begin{array}{c}
k-1 \\
j-1
\end{array}\right),
$$

for all $k \in \mathbb{N}$ and $\mathbb{P}(N([0, t))=0)=\mathrm{e}^{-\theta t}$.

When $D^{\prime}\left(u_{n}\right)$ holds, since there is no clustering, then, due to a criterion proposed by Kallenberg [18, Theorem 4.7], which applies only to simple point processes, without multiple events, we can simply adjust condition $D_{2}\left(u_{n}\right)$ to this scenario of multiple exceedances in order to prove that the REPP converges in distribution to a standard Poisson process. We denote this adapted condition by:

Condition $\left(D_{3}\left(u_{n}\right)\right)$. Let $A \in \mathcal{R}$ and $t \in \mathbb{N}$. We say that $D_{3}\left(u_{n}\right)$ holds for the sequence $X_{0}, X_{1}, \ldots$ if

$$
\left|\mathbb{P}\left(\left\{X_{0}>u_{n}\right\} \cap\{\mathscr{N}(A+t)=0\}\right)-\mathbb{P}\left(\left\{X_{0}>u_{n}\right\}\right) \mathbb{P}(\mathscr{N}(A)=0)\right| \leq \gamma(n, t),
$$

where $\gamma(n, t)$ is non-increasing in $t$ for each $n$ and $n \gamma\left(n, t_{n}\right) \rightarrow 0$ as $n \rightarrow \infty$ for some sequence $t_{n}=o(n)$, which means that $t_{n} / n \rightarrow 0$ as $n \rightarrow \infty$.

Condition $D_{3}\left(u_{n}\right)$ follows, as easily as $D_{2}\left(u_{n}\right)$, from sufficiently fast decay of correlations.

In [10, Theorem 5] a strengthening of [9, Theorem 1] is proved, which essentially says that, under $D_{3}\left(u_{n}\right)$ and $D^{\prime}\left(u_{n}\right)$, the REPP $N_{n}$ defined in (2.20) converges in distribution to a standard Poisson process.

Next, we give an abstract result, in the deterministic setting, that allows us to check conditions $D_{2}\left(u_{n}\right)$ and $D^{\prime}\left(u_{n}\right)$ for any stochastic process $X_{0}, X_{1}, \ldots$ arising from a system which has decay of correlations against $L^{1}$ observables. As a consequence of this result in Section 3 more precisely in Propositions 3.2 3.3 and 3.4 . we will obtain the announced dichotomy for the EI based on the periodicity of the point $\zeta$.

Theorem A. Consider a dynamical system $(\mathcal{M}, \mathcal{B}, \mu, f)$ for which there exists a Banach space $\mathcal{C}$ of real valued functions such that for all $\phi \in \mathcal{C}$ and $\psi \in L^{1}(\mu)$,

$$
\operatorname{Cor}_{\mu}(\phi, \psi, n) \leq \mathrm{Cn}^{-2},
$$

where $C>0$ is a constant independent of both $\phi, \psi$. Let $X_{0}, X_{1}, \ldots$ be given by (2.6), where $\varphi$ achieves a global maximum at some point $\zeta$ for which condition (R1) holds. Let $u_{n}$ be such that (2.10) holds, $U_{n}$ be defined as in (2.12) and set $R_{n}:=R\left(U_{n}\right)$.

If there exists $C^{\prime}>0$ such that for all $n$ we have $\mathbf{1}_{U_{n}} \in \mathcal{C},\left\|\mathbf{1}_{U_{n}}\right\|_{\mathcal{C}} \leq C^{\prime}$ and $R_{n} \rightarrow \infty$, as $n \rightarrow \infty$, then conditions $D_{2}\left(u_{n}\right)$ and $D^{\prime}\left(u_{n}\right)$ hold for $X_{0}, X_{1}, \ldots$ This implies that there is an EVL for $M_{n}$ defined in (2.9) and $H(\tau)=1-\mathrm{e}^{-\tau}$.

In light of the connection between EVLs and HTS/RTS it follows immediately:

Corollary B. Under the same hypothesis as Theorem $\mathrm{A}$ we have HTS/RTS for balls around $\zeta$ with $G(t)=\tilde{G}(t)=1-\mathrm{e}^{-t}$.

Since, under the same assumptions of Theorem $\mathrm{A}$, condition $D_{3}\left(u_{n}\right)$ holds trivially, then applying [10, Theorem 5] we obtain the following corollary. 
Corollary C. Under the same hypothesis as Theorem $\mathrm{A}$, the REPP $N_{n}$ defined in (2.20) is such that $N_{n} \stackrel{d}{\rightarrow} N$, as $n \rightarrow \infty$, where $N$ denotes a Poisson Process with intensity 1.

Remark 2.4. Note that condition $R_{n} \rightarrow \infty$, as $n \rightarrow \infty$, is easily verified if the map is continuous at every point of the orbit of $\zeta$. We will state this formally in Lemma 3.1

Remark 2.5. Observe that decay of correlations as in (2.21) against $L^{1}(\mu)$ observables is a very strong property. In fact, regardless of the rate (in this case $n^{-2}$ ), as long as it is summable, one can actually show that the system has exponential decay of correlations of Hölder observables against $L^{\infty}(\mu)$, i.e., $\mathcal{C}_{1}$ is the space of Hölder continuous and $\mathcal{C}_{2}$ is $L^{\infty}(\mu)$. (See [1, Theorem B].)

Now, we give an abstract result in the random setting which concludes by stating that by adding random noise, regardless of the point $\zeta$ chosen, we always get an EI equal to 1.

Theorem D. Consider a dynamical system $\left(\mathcal{M} \times \Omega, \mathcal{B}, \mu_{\varepsilon} \times \theta_{\varepsilon}^{\mathbb{N}}, S\right)$, where $\mathcal{M}=$ $\mathbb{T}^{d}$, for some $d \in \mathbb{N}, f: \mathcal{M} \rightarrow \mathcal{M}$ is a deterministic system which is randomly perturbed as in (2.2) with noise distribution given by (2.1) and $S$ is the skew product map defined in (2.4). Assume that there exists $\eta>0$ such that $\operatorname{dist}(f(x), f(y)) \leq$ $\eta \operatorname{dist}(x, y)$, for all $x, y \in \mathcal{M}$. Assume also that the stationary measure $\mu_{\varepsilon}$ is such that $\mu_{\varepsilon}=h_{\varepsilon}$ Leb, with $0<\underline{h}_{\varepsilon} \leq h_{\varepsilon} \leq \bar{h}_{\varepsilon}<\infty$. Suppose that there exists a Banach space $\mathcal{C}$ of real valued functions defined on $\mathcal{M}$ such that for all $\phi \in \mathcal{C}$ and $\psi \in L^{1}\left(\mu_{\varepsilon}\right)$,

$$
\operatorname{Cor}_{\mu_{\varepsilon} \times \theta_{\varepsilon}^{\mathbb{N}}}(\phi, \psi, n) \leq C n^{-2},
$$

where $\operatorname{Cor}_{\mu_{\varepsilon} \times \theta_{\varepsilon}^{\mathbb{N}}}(\cdot)$ is defined as in (2.5) and $C>0$ is a constant independent of both $\phi, \psi$.

For any point $\zeta \in \mathcal{M}$, consider that $X_{0}, X_{1}, \ldots$ is defined as in (2.7), let $u_{n}$ be such that (2.10) holds and assume that $U_{n}$ is defined as in (2.12). If there exists $C^{\prime}>0$ such that for all $n$ we have $\mathbf{1}_{U_{n}} \in \mathcal{C}$ and $\left\|\mathbf{1}_{U_{n}}\right\|_{\mathcal{C}} \leq C^{\prime}$, then the stochastic process $X_{0}, X_{1}, \ldots$ satisfies $D_{2}\left(u_{n}\right)$ and $D^{\prime}\left(u_{n}\right)$, which implies that we have an $E V L$ for $M_{n}$ such that $\bar{H}(\tau)=\mathrm{e}^{-\tau}$.

Again, using the connection between EVLs and HTS/RTS we get

Corollary E. Under the same hypothesis as Theorem $\mathrm{D}$, we have exponential HTS/RTS for balls around $\zeta$, in the sense that (2.15) and (2.16) hold with $G(t)=$ $\tilde{G}(t)=1-\mathrm{e}^{-t}$ and $V_{n}=B_{\delta_{n}}(\zeta) \times \Omega$, where $\delta_{n} \rightarrow 0$, as $n \rightarrow \infty$.

Moreover, appealing to [10, Theorem 5] once again, we have

Corollary F. Under the same hypothesis as Theorem D, the stochastic process $X_{0}, X_{1}, \ldots$ satisfies $D_{3}\left(u_{n}\right)$ and $D^{\prime}\left(u_{n}\right)$, which implies that the REPP $N_{n}$ defined in (2.20) is such that $N_{n} \stackrel{d}{\rightarrow} N$, as $n \rightarrow \infty$, where $N$ denotes a Poisson Process with intensity 1.

Remark 2.6. We remark that we do not need to consider that $\mathcal{M}$ is a $d$-dimensional torus in order to apply the theory. Basically, we only need that $f_{\omega}(\mathcal{M}) \subset \mathcal{M}$, for all $\omega \in B_{\varepsilon}(0)$. As we will see in more detail in Section 4 , for example, piecewise expanding maps of the interval, with finitely many branches, satisfy all the conclusions of Theorem D. 


\section{Extremal Index Dichotomy FOR Deterministic Systems}

In this section we will start by proving Theorem $\mathrm{A}$, Corollary $\mathrm{C}$ and a simple lemma asserting that continuity is enough to guarantee that $R_{n} \rightarrow \infty$, as $n \rightarrow \infty$.

Next, we give examples of systems to which we can apply Theorem $\mathrm{A}$ in order to prove a dichotomy regarding the existence of an EI equal to 1 or less than 1, depending on whether $\zeta$ is non-periodic or periodic, respectively. This will be done for uniformly expanding and piecewise expanding maps, when all points in the orbit of $\zeta$ are continuity points of the map.

In the third subsection, we will consider Rychlik maps, which are piecewise expanding maps of the interval, and will analyse the EI also in the cases when the orbit of $\zeta$ hits a discontinuity point of the map.

\subsection{Decay against $L^{1}$ implies exponential EVL at non-periodic points.}

Proofs of Theorem $\mathrm{A}$ and Corollary C. As explained in [14, Section 5.1], conditions $D_{2}\left(u_{n}\right)$ and $D_{3}\left(u_{n}\right)$ are designed to follow easily from decay of correlations. In fact, if we choose $\phi=\mathbf{1}_{U_{n}}$ and $\psi=\mathbf{1}_{\left\{M_{\ell} \leq u_{n}\right\}}$, in the case of $D_{2}\left(u_{n}\right)$, and $\psi=\mathbf{1}_{\mathscr{N}(A)=0}$, for some $A \in \mathcal{R}$, in the case of $\bar{D}_{3}\left(u_{n}\right)$, we have that we can take $\gamma(n, t)=C^{*} t^{-2}$, where $C^{*}=C C^{\prime}$. Hence, conditions $D_{2}\left(u_{n}\right)$ and $D_{3}\left(u_{n}\right)$ are trivially satisfied for the sequence $\left(t_{n}\right)_{n}$ given by $t_{n}=n^{2 / 3}$, for example.

Now, we turn to condition $D^{\prime}\left(u_{n}\right)$. Taking $\psi=\phi=\mathbf{1}_{U_{n}}$ in (2.21) and since $\left\|\mathbf{1}_{U_{n}}\right\|_{\mathcal{C}} \leq C^{\prime}$, we easily get

$\mu\left(U_{n} \cap f^{-j}\left(U_{n}\right)\right) \leq\left(\mu\left(U_{n}\right)\right)^{2}+C\left\|\mathbf{1}_{U_{n}}\right\|_{\mathcal{C}}\left\|\mathbf{1}_{U_{n}}\right\|_{L^{1}(\mu)} j^{-2} \leq\left(\mu\left(U_{n}\right)\right)^{2}+C^{*} \mu\left(U_{n}\right) j^{-2}$,

where $C^{*}=C C^{\prime}>0$. By definition of $R_{n}$, estimate (3.1) and since $n \mu\left(U_{n}\right) \rightarrow \tau$ as $n \rightarrow \infty$, it follows that there exists some constant $D>0$ such that

$$
\begin{aligned}
n \sum_{j=1}^{\left\lfloor n / k_{n}\right\rfloor} \mu & \left(U_{n} \cap f^{-j}\left(U_{n}\right)\right) \\
& =n \sum_{j=R_{n}}^{\left\lfloor n / k_{n}\right\rfloor} \mu\left(U_{n} \cap f^{-j}\left(U_{n}\right)\right) \leq n\left\lfloor\frac{n}{k_{n}}\right\rfloor \mu\left(U_{n}\right)^{2}+n C^{*} \mu\left(U_{n}\right) \sum_{j=R_{n}}^{\left\lfloor n / k_{n}\right\rfloor} j^{-2} \\
& \leq \frac{\left(n \mu\left(U_{n}\right)\right)^{2}}{k_{n}}+n C^{*} \mu\left(U_{n}\right) \sum_{j=R_{n}}^{\infty} j^{-2} \leq D\left(\frac{\tau^{2}}{k_{n}}+\tau \sum_{j=R_{n}}^{\infty} j^{-2}\right) \underset{n \rightarrow \infty}{\longrightarrow} 0 .
\end{aligned}
$$

Remark 3.1. In the above demonstration it is important to use the $L^{1}$-norm to obtain the factor $\mu\left(U_{n}\right)$ in the second summand of the last term in (3.1), which is crucial to kill off the $n$ factor coming from the definition of $D^{\prime}\left(u_{n}\right)$. However, note that we actually do not need decay of correlations against $L^{1}$ in its full strength, which means that it holds for all $L^{1}$ functions. In fact, in order to prove $D^{\prime}\left(u_{n}\right)$ we only need it to hold for the functions $\mathbf{1}_{U_{n}}$.

Also, note that we do not need such a strong statement regarding the decay of correlations of the system in order to prove $D_{2}\left(u_{n}\right)$ or $D_{3}\left(u_{n}\right)$. In particular, even if $\mathbf{1}_{U_{n}} \notin \mathcal{C}$ (as when $\mathcal{C}$ is the space of Hölder continuous functions), we can 
still verify these conditions by using a suitable Hölder approximation. (See 14, Proposition 5.2].)

According to Theorem $\mathrm{A}$, in general terms, if the system has decay of correlations against $L^{1}$ observables, then to prove $D^{\prime}\left(u_{n}\right)$ one has basically to show that $R_{n} \rightarrow$ $\infty$, as $n \rightarrow \infty$. Next lemma gives us a sufficient condition for that to happen.

Lemma 3.1. Assume that $\zeta$ is not a periodic point and that $f$ is continuous at every point of the orbit of $\zeta$, namely $\zeta, f(\zeta), f^{2}(\zeta), \ldots$ Then $\lim _{n \rightarrow \infty} R_{n}=\infty$, where $R_{n}$ is as in Theorem $\mathrm{A}$.

Proof. Let $j \in \mathbb{N}$. We will show that if $n \in \mathbb{N}$ is sufficiently large, then $R_{n}>j$. Let $\epsilon=\min _{i=1, \ldots, j} \operatorname{dist}\left(f^{i}(\zeta), \zeta\right)$. Our assumptions assure that each $f^{i}$, for $i=1, \ldots, j$, is continuous at $\zeta$. Hence, for every $i=1, \ldots, j$, there exists $\delta_{i}>0$ such that $f^{i}\left(B_{\delta_{i}}(\zeta)\right) \subset B_{\epsilon / 2}\left(f^{i}(\zeta)\right)$. Let $U:=\bigcap_{i=1}^{j} B_{\delta_{i}}(\zeta)$. If we choose $N$ sufficiently large that $U_{n} \subset U$ for all $n \geq N$, then using the definition of $\epsilon$ it is clear that $f^{i}\left(U_{n}\right) \cap U_{n}=\emptyset$, for all $i=1, \ldots, j$, which implies that $R_{n}>j$.

3.2. The dichotomy for specific systems. One of the results in 12 is that for uniformly expanding systems like the doubling map, there is a dichotomy in terms of the type of laws of rare events that one gets at every possible centre $\zeta$. Namely, it was shown that either $\zeta$ is non-periodic, in which case one always gets a standard exponential EVL/HTS, or $\zeta$ is a periodic (repelling) point, in which case one obtains an exponential law with an EI $0<\vartheta<1$ given by the expansion rate at $\zeta$ (see [12, Section 6]). This was proved for cylinders rather than balls, meaning that the sets $U_{n}$ are dynamically defined cylinders (see [12, Section 5] or [11, Section 5], for details). Results for cylinders are weaker than the ones for balls, since, in rough terms, it means that the limit is only obtained for certain subsequences of $n \in \mathbb{N}$ rather than for the whole sequence.

In [12, it was conjectured that this dichotomy should hold in greater generality, namely for balls rather than cylinders and more general systems. As a consequence of Theorem $\mathrm{A}$ we will be able to show that the dichotomy indeed holds for balls and more general systems. We remark that from the results in 8 , one can also derive the dichotomy for conformal repellers and, in [20], the dichotomy is also obtained for maps with a spectral gap for their Perron-Frobenius operator. In both these papers, the results were obtained by studying the spectral properties of the Perron-Frobenius operator.

3.2.1. Rychlik maps. We will introduce a class of dynamical systems considered by Rychlik in [27]. This class includes, for example, piecewise $C^{2}$ uniformly expanding maps of the unit interval with the relevant physical measures. We first need some definitions.

Definition 3.1. Given a potential $\psi: Y \rightarrow \mathbb{R}$ on an interval $Y$, the variation of $\psi$ is defined as

$$
\operatorname{Var}(\psi):=\sup \left\{\sum_{i=0}^{n-1}\left|\psi\left(x_{i+1}\right)-\psi\left(x_{i}\right)\right|\right\},
$$

where the supremum is taken over all finite ordered sequences $\left(x_{i}\right)_{i=0}^{n} \subset Y$.

We use the norm $\|\psi\|_{B V}=\sup |\psi|+\operatorname{Var}(\psi)$, which makes $B V:=\{\psi: Y \rightarrow \mathbb{R}:$ $\left.\|\psi\|_{B V}<\infty\right\}$ into a Banach space. We also define

$$
S_{n} \psi(x):=\psi(x)+\cdots+\psi \circ f^{n-1}(x) .
$$


Definition 3.2. For a measurable potential $\phi: \mathcal{X} \rightarrow \mathbb{R}$, we define the pressure of $(\mathcal{X}, f, \phi)$ to be

$$
P(\phi):=\sup _{\mathbb{P} \in \mathcal{M}_{f}}\left\{h_{\mathbb{P}}(f)+\int \phi d \mathbb{P}:-\int \phi d \mathbb{P}<\infty\right\},
$$

where $\mathcal{M}_{f}$ is the set of $f$-invariant probability measures and $h_{\mathbb{P}}(f)$ denotes the metric entropy of $f$ w.r.t. the measure $\mathbb{P}$; see 31 for details. If $\mathbb{P}$ is an invariant probability measure such that $h\left(\mathbb{P}_{\phi}\right)+\int \phi d \mathbb{P}=P(\phi)$, then we say that $\mathbb{P}$ is an equilibrium state.

Definition 3.3. A measure $m$ is called a $\phi$-conformal measure if $m(\mathcal{M})=1$ and if whenever $f: A \rightarrow f(A)$ is a bijection, for a Borel set $A$, then $m(f(A))=\int_{A} e^{-\phi} d m$. Therefore, if $f^{n}: A \rightarrow f^{n}(A)$ is a bijection, then $m\left(f^{n}(A)\right)=\int_{A} e^{-S_{n} \phi} d m$.

Definition 3.4 (Rychlik system). $(Y, f, \psi)$ is a Rychlik system if $Y$ is an interval, $\left\{Y_{i}\right\}_{i}$ is an at most countable collection of open intervals such that $\bigcup_{i} \bar{Y}_{i} \supset Y$ (where $\bar{Y}_{i}$ is the closure of $Y_{i}$ ), $f: \bigcup_{i} Y_{i} \rightarrow Y$ is a function continuous on each $Y_{i}$, which admits a continuous extension to the closure of $Y_{i}$ that we denote by $f_{i}: \bar{Y}_{i} \rightarrow Y$ and $\psi: Y \rightarrow[-\infty, \infty)$ is a potential such that

(1) $f_{i}: \bar{Y}_{i} \rightarrow f\left(\bar{Y}_{i}\right)$ is a diffeomorphism;

(2) Var $\mathrm{e}^{\psi}<+\infty, \psi=-\infty$ on $Y \backslash \bigcup_{i} Y_{i}$ and $P(\psi)=0$;

(3) there is a $\psi$-conformal measure $m_{\psi}$ on $Y$;

(4) $(f, \psi)$ is expanding: $\sup _{x \in Y} \psi(x)<0$.

Rychlik 27] proved that these maps have exponential decay of correlations against $L^{1}$ observables. To be more precise, if $(Y, f, \psi)$ is a topologically mixing Rychlik system, then there exists an equilibrium state $\mu_{\psi}=h m_{\psi}$ where $h \in B V$ and $m_{\psi}$ and $\mu_{\psi}$ are non-atomic and $\left(Y, f, \mu_{\psi}\right)$ has exponential decay of correlations, i.e., there exists $C>0$ and $\gamma \in(0,1)$ such that

$$
\left|\int \varsigma \circ f^{n} \cdot \phi \mathrm{d} \mu_{\psi}-\int \varsigma \mathrm{d} \mu_{\psi} \int \phi \mathrm{d} \mu_{\psi}\right| \leq C\|\varsigma\|_{L^{1}\left(\mu_{\psi}\right)}\|\phi\|_{B V} \gamma^{n},
$$

for any $\varsigma \in L^{1}\left(\mu_{\psi}\right)$ and $\phi \in B V$. Note that, in the original statement, instead of the $L^{1}\left(\mu_{\psi}\right)$-norm, the $L^{1}\left(m_{\psi}\right)$-norm appeared. However, we will assume that $h>c$, for some $c>0$, which means that we can write (3.2) as it is. We remark that $h$ being bounded below by a positive constant is not very restrictive. That is the case if, for example, $h$ is lower semi-continuous (see [5, Theorem 8.2.3]) or if the system has summable variations as does uniformly expanding systems with Hölder continuous potentials.

Let $\mathbb{S}=Y \backslash \bigcup_{i} Y_{i}$ and define $\Lambda:=\left\{x \in Y: f^{n}(x) \notin \mathbb{S}\right.$, for all $\left.n \in \mathbb{N}_{0}\right\}$. As a consequence of Theorem $\mathrm{A}$, Corollary $\mathrm{C}$ and Lemma 3.1 it follows immediately:

Proposition 3.2. Suppose that $(Y, f, \psi)$ is a topologically mixing Rychlik system, $\psi$ is Hölder continuous on each $\bar{Y}_{i}$, and $\mu=\mu_{\psi}$ is the corresponding equilibrium state such that $\frac{d \mu_{\psi}}{d m_{\psi}}>c$, for some $c>0$. Let $X_{0}, X_{1}, \ldots$ be given by (2.6), where $\varphi$ achieves a global maximum at some point $\zeta$. Then we have an $E V L$ for $M_{n}$ and

(1) if $\zeta \in \Lambda$ is not a periodic point, then the $E V L$ is such that $\bar{H}(\tau)=\mathrm{e}^{-\tau}$ and the REPP $N_{n}$ converges in distribution to a standard Poisson process $N$ of intensity 1 ; 
(2) if $\zeta \in \Lambda$ is a (repelling) periodic point of prime period p, then the EVL is such that $\bar{H}(\tau)=\mathrm{e}^{-\vartheta \tau}$, where the EI is given by $\vartheta=1-\mathrm{e}^{S_{p} \psi(\zeta)}$ and the REPP $N_{n}$ converges in distribution to a compound Poisson process $N$ with intensity $\vartheta$ and multiplicity d.f. $\pi$ given by $\pi(\kappa)=\vartheta(1-\vartheta)^{\kappa}$, for every $\kappa \in \mathbb{N}_{0}$.

Proof. We start by noting that statement (2) has already been proved in [12, Proposition 2] and [13, Corollary 3].

Regarding statement (1), first note that for Rychlik maps, (3.2) clearly implies that condition (2.21) is satisfied. Besides, since $U_{n}$ must be an interval, then $\mathbf{1}_{U_{n}} \in B V$ and $\left\|\mathbf{1}_{U_{n}}\right\|_{B V} \leq 2$. Moreover, by definition of $\Lambda$, we can apply Lemma 3.1 and consequently obtain that $\lim _{n \rightarrow \infty} R_{n}=\infty$. Hence, we are now in a condition of applying Theorem $\mathrm{A}$ and Corollary $\mathrm{C}$ to obtain the result.

3.2.2. Piecewise expanding maps in higher dimensions. As a second example, we will consider multidimensional piecewise uniformly expanding maps for which we follow the definition given by Saussol 28. As pointed out in [1, these maps generalise Markov maps which also contain one-dimensional piecewise uniformly expanding maps.

We need some notation: $\operatorname{dist}(\cdot, \cdot)$ being the usual metric in $\mathbb{R}^{N}, N \in \mathbb{N}$, given any $\varepsilon>0$, we introduce $B_{\varepsilon}(x)=\left\{y \in \mathbb{R}^{N}\right.$ : $\left.\operatorname{dist}(x, y)<\varepsilon\right\}$. Moreover, $Z$ being a compact subset of $\mathbb{R}^{N}$, for any $A \subset Z$ and given a real number $c>0$, we write $B_{c}(A)=\left\{x \in \mathbb{R}^{N}: \operatorname{dist}(x, A) \leq c\right\} ; Z^{\circ}$ stands for the interior of $Z$, and $\bar{Z}$ is the closure.

Definition 3.5 (Multidimensional piecewise expanding system). $(Z, f, \mu)$ is a multidimensional piecewise expanding system if $Z$ is a compact subset of $\mathbb{R}^{N}$ with $\overline{Z^{\circ}}=Z, f: Z \rightarrow Z$ and $\left\{Z_{i}\right\}$ is a family of at most countably many disjoint open sets such that $\operatorname{Leb}\left(Z \backslash \bigcup_{i} Z_{i}\right)=0$ and there exist open sets $\widetilde{Z_{i}} \supset \overline{Z_{i}}$ and $C^{1+\alpha}$ maps $f_{i}: \widetilde{Z}_{i} \rightarrow \mathbb{R}^{N}$, for some real number $0<\alpha \leq 1$ and some sufficiently small real number $\varepsilon_{1}>0$ such that for all $i$,

(1) $f_{i}\left(\widetilde{Z_{i}}\right) \supset B_{\varepsilon_{1}}\left(f\left(Z_{i}\right)\right)$;

(2) for $x, y \in f\left(Z_{i}\right)$ with $\operatorname{dist}(x, y) \leq \varepsilon_{1}$,

$$
\left|\operatorname{det} D f_{i}^{-1}(x)-\operatorname{det} D f_{i}^{-1}(y)\right| \leq c\left|\operatorname{det} D f_{i}^{-1}(x)\right| \operatorname{dist}(x, y)^{\alpha} \text {; }
$$

(3) there exists $s=s(f)<1$ such that $\forall x, y \in f\left(\widetilde{Z_{i}}\right)$ with $\operatorname{dist}(x, y) \leq \varepsilon_{1}$, we have

$$
\operatorname{dist}\left(f_{i}^{-1} x, f_{i}^{-1} y\right) \leq s \operatorname{dist}(x, y)
$$

(4) let us put $G\left(\varepsilon, \varepsilon_{1}\right):=\sup _{x} G\left(x, \varepsilon, \varepsilon_{1}\right)$, where

$$
G\left(x, \varepsilon, \varepsilon_{1}\right):=\sum_{i} \frac{\operatorname{Leb}\left(f_{i}^{-1} B_{\varepsilon}\left(\partial f Z_{i}\right) \cup B_{(1-s) \varepsilon_{1}}(x)\right)}{\operatorname{Leb}\left(B_{(1-s) \varepsilon_{1}}(x)\right)}
$$

and assume that $\sup _{\delta \leq \varepsilon_{1}}\left(s^{\alpha}+2 \sup _{\varepsilon \leq \delta} \frac{G(\varepsilon)}{\varepsilon^{\alpha}} \delta^{\alpha}\right)<1$.

Now, let us introduce the space of quasi-Hölder functions in which the spectrum of the corresponding Perron-Frobenius operator is investigated. Given a Borel set $\Gamma \subset Z$, we define the oscillation of $\varphi \in L^{1}$ (Leb) over $\Gamma$ as

$$
\operatorname{osc}(\varphi, \Gamma):=\underset{\Gamma}{\operatorname{ess} \sup } \varphi-\underset{\Gamma}{\operatorname{ess} \inf } \varphi \text {. }
$$


It is easy to verify that $x \mapsto \operatorname{osc}\left(\varphi, B_{\varepsilon}(x)\right)$ defines a measurable function (see [28, Proposition 3.1]). Given real numbers $0<\alpha \leq 1$ and $\varepsilon_{0}>0$, we define the $\alpha$-seminorm of $\varphi$ as

$$
|\varphi|_{\alpha}=\sup _{0<\varepsilon \leq \varepsilon_{0}} \varepsilon^{-\alpha} \int_{\mathbb{R}^{\mathbb{N}}} \operatorname{osc}\left(\varphi, B_{\varepsilon}(x)\right) \operatorname{dLeb}(x) .
$$

Let us consider the space of functions with bounded $\alpha$-seminorm,

$$
V_{\alpha}=\left\{\varphi \in L^{1}(\mathrm{Leb}):|\varphi|_{\alpha}<\infty\right\},
$$

and endow $V_{\alpha}$ with the norm

$$
\|\cdot\|_{\alpha}=\|\cdot\|_{L^{1}(\mathrm{Leb})}+|\cdot|_{\alpha}
$$

which makes it into a Banach space. We note that $V_{\alpha}$ is independent of the choice of $\varepsilon_{0}$. According to [28, Theorem 5.1], there exists an absolutely continuous invariant probability measure (a.c.i.p.) $\mu$. Also in [28, Theorem 6.1], it is shown that on the mixing components $\mu$ enjoys exponential decay of correlations against $L^{1}$ observables on $V_{\alpha}$. More precisely, if the map $f$ is as defined above and if $\mu$ is the mixing a.c.i.p., then there exist constants $C<\infty$ and $\gamma<1$ such that

$$
\left|\int_{Z} \psi \circ f^{n} h \mathrm{~d} \mu\right| \leq C\|\psi\|_{L^{1}}\|h\|_{\alpha} \gamma^{n}, \forall \psi \in L^{1} \text {, where } \int \psi \mathrm{d} \mu=0 \text { and } \forall h \in V_{\alpha} .
$$

We refer the reader to [28] for the exact values of the above constants. We point out that the $L^{1}$-norm on the right hand side should be intended with respect to Leb. Whenever the density of $\mu$ is bounded from below and Lebesgue almost everywhere by a strictly positive constant, such an $L^{1}$-norm could be extended to $\mu$; see also Appendix C.4 in [1] for a similar derivation.

Let $\mathbb{S}=Z \backslash \bigcup_{i} Z_{i}$ and define $\Lambda:=\left\{x \in Z: f^{n}(x) \notin \mathbb{S}\right.$, for all $\left.n \in \mathbb{N}_{0}\right\}$. As a consequence of Theorem $\mathrm{A}$. Corollary $\mathrm{C}$ and Lemma 3.1 it follows immediately that:

Proposition 3.3. Suppose that $(Z, f, \mu)$ is a topologically mixing multidimensional piecewise expanding system and $\mu$ is its a.c.i.p. with a Radon-Nikodym density bounded away from zero. Let $X_{0}, X_{1}, \ldots$ be given by (2.6), where $\varphi$ achieves a global maximum at some point $\zeta$. Then we have an $E V L$ for $M_{n}$ and

(1) if $\zeta \in \Lambda$ is not a periodic point, then the $E V L$ is such that $\bar{H}(\tau)=\mathrm{e}^{-\tau}$ and the REPP $N_{n}$ converges in distribution to a standard Poisson process $N$ of intensity 1 ;

(2) if $\zeta \in \Lambda$ is a (repelling) periodic point of prime period p, then the EVL is such that $\bar{H}(\tau)=\mathrm{e}^{-\vartheta \tau}$, where the EI is given by $\vartheta=1-\left|\operatorname{det} D\left(f^{-p}\right)(\zeta)\right|$ and the REPP $N_{n}$ converges in distribution to a compound Poisson process $N$ with intensity $\vartheta$ and multiplicity d.f. $\pi$ given by $\pi(\kappa)=\vartheta(1-\vartheta)^{\kappa}$, for every $\kappa \in \mathbb{N}_{0}$.

Proof. Statement (2) has already been proved in [13, Corollary 4].

For proving (1), we can start by remarking that condition (2.21) is satisfied since we have (3.4). Since $U_{n}$ corresponds to a ball, by definition of $|\cdot|_{\alpha}$, it follows easily that $\mathbf{1}_{U_{n}} \in V_{\alpha}$ and $\left\|\mathbf{1}_{U_{n}}\right\|_{\alpha}$ is uniformly bounded by above. Now, 
considering the definition of $\Lambda$, we can apply Lemma 3.1 and consequently obtain that $\lim _{n \rightarrow \infty} R_{n}=\infty$. The result then follows by applying Theorem $\mathrm{A}$ and Corollary C.

3.3. The extremal behaviour at discontinuity points. In this section, we go back to Rychlik maps introduced in Section 3.2.1, but with finitely many branches, and study the extremal behaviour of the system when the orbit of $\zeta$ hits a discontinuity point of the map.

Consider a point $\zeta \in Y$. Note that here we consider at most finitely many collections of open intervals such that $\bigcup_{i} \bar{Y}_{i} \supset Y$. If $\zeta \in \Lambda$, then we say that $\zeta$ is a simple point. If $\zeta$ is a non-simple point, which means that $r_{\mathbb{S}}(\zeta)$ is finite, then let $\ell=r_{\mathbb{S}}(\zeta)$ and $z=f^{\ell}(\zeta)$. We will always assume that $z \in \mathbb{S}$ is such that: there exist $i^{+}, i^{-} \in \mathbb{N}$ so that $z$ is the right end point of $Y_{i^{-}}$and the left end point of $Y_{i^{+}}$. We consider that the point $z$ is doubled and has two versions, $z^{+} \in Y_{i^{+}}$and $z^{-} \in Y_{i^{-}}$, so that

$$
f\left(z^{+}\right):=f_{i^{+}}(z)=\lim _{x \rightarrow z, x \in Y_{i^{+}}} f(x) \text { and } f\left(z^{-}\right):=f_{i^{-}}(z)=\lim _{x \rightarrow z, x \in Y_{i^{-}}} f(x) .
$$

When $\zeta$ is a non-simple point we consider that its orbit bifurcates when it hits $\mathbb{S}$ and consider its two possible evolutions. We express this fact by saying that when $\zeta$ is non-simple we consider the "orbits" of $\zeta^{+}$and $\zeta^{-}$which are defined in the following way:

- for $j=1, \ldots, \ell$ we let $f^{j}\left(\zeta^{ \pm}\right):=f^{j}(\zeta)$;

- for $j=\ell+1$, we define $f^{j}\left(\zeta^{ \pm}\right):=f_{i \pm}\left(f^{j-1}\left(\zeta^{ \pm}\right)\right)$;

- for $j>\ell+1$ we consider two possibilities:

- if $j-1$ is such that $f^{j-1}\left(\zeta^{ \pm}\right) \notin \mathbb{S}$, then we set $f^{j}\left(\zeta^{ \pm}\right):=f\left(f^{j-1}\left(\zeta^{ \pm}\right)\right)$,

- otherwise we set $f^{j}\left(\zeta^{ \pm}\right):=f_{i}\left(f^{j-1}\left(\zeta^{ \pm}\right)\right)$, where $i$ is such that $f^{j-\ell}\left(z^{ \pm}\right)$ $\in Y_{i}$.

Remark 3.2. Note that for the "orbits" of $\zeta^{ \pm}$just defined above, there is a sequence $\left(i_{j}^{ \pm}\right)_{j \in \mathbb{N}}$ such that, for all $n \in \mathbb{N}$, we have $f^{n}\left(\zeta^{ \pm}\right) \in \bar{Y}_{i_{n}^{ \pm}}$and $f^{n}\left(\zeta^{ \pm}\right)=f_{i_{n}^{ \pm}} \circ \cdots \circ$ $f_{i_{1}^{ \pm}}(\zeta)$. Also observe that, in the notation above, $i_{\ell}^{ \pm}=i^{ \pm}$.

A non-simple point $\zeta$ is aperiodic if for all $j \in \mathbb{N}$ we have $f^{j}\left(\zeta^{+}\right) \neq \zeta \neq f^{j}\left(\zeta^{-}\right)$.

If there exists $p^{ \pm}$such that $f^{p^{ \pm}}\left(\zeta^{ \pm}\right)=\zeta$ and for $j=1, \ldots, p^{ \pm}-1$ we have $f^{j}\left(\zeta^{ \pm}\right) \neq \zeta$, but, for all $j \in \mathbb{N}$, we have $f^{j}\left(\zeta^{\mp}\right) \neq \zeta$, then we say that $\zeta$ is singly returning. If $\zeta$ is singly returning and $f^{ \pm}\left(\zeta^{ \pm}\right)=\zeta^{ \pm}$, which means that $f^{p^{ \pm}}\left(z^{ \pm}\right) \in$ $Y_{i^{ \pm}}$, then we say that $\zeta$ is a singly periodic point of period $p^{ \pm}$.

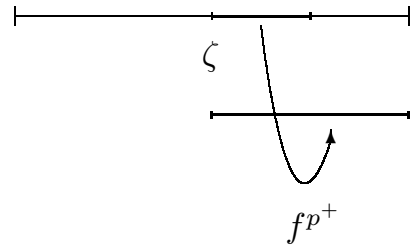

FiguRE 1. Singly returning, singly periodic $\zeta$ 
If $\zeta$ is singly returning and $f^{p^{ \pm}}\left(\zeta^{ \pm}\right)=\zeta^{\mp}$, which means that $f^{p^{ \pm}}\left(z^{ \pm}\right) \in Y_{i^{\mp}}$, then we say that $\zeta$ is an eventually aperiodic point.

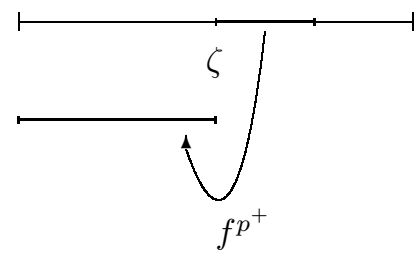

FIGURE 2. Singly returning, eventually aperiodic $\zeta$

If there exist $p^{+}$and $p^{-}$such that $f^{p^{+}}\left(\zeta^{+}\right)=\zeta=f^{p^{-}}\left(\zeta^{-}\right)$and for $j=1, \ldots$, $p^{+}-1$ and $k=1, \ldots, p^{-}-1$ we have $f^{j}\left(\zeta^{+}\right) \neq \zeta \neq f^{k}\left(\zeta^{-}\right)$, then we say that $\zeta$ is doubly returning. In the case that $\zeta$ is a doubly returning point and both $f^{p^{+}}\left(\zeta^{+}\right)=\zeta^{+}$and $f^{p^{-}}\left(\zeta^{-}\right)=\zeta^{-}$, we say that $\zeta$ is doubly periodic with periods $p^{+}$ and $p^{-}$, respectively.

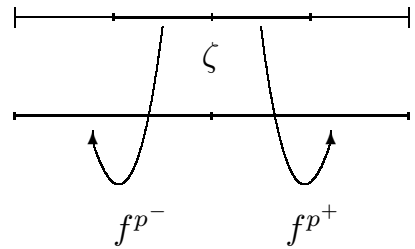

FIGURE 3. Doubly returning, doubly periodic $\zeta$ (no switches)

If $\zeta$ is doubly returning, $f^{p^{ \pm}}\left(\zeta^{ \pm}\right)=\zeta^{ \pm}$and $f^{p^{\mp}}\left(\zeta^{\mp}\right)=\zeta^{ \pm}$, then we say that $\zeta$ is doubly returning with one switch.

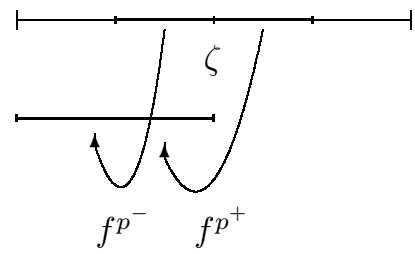

FiguRE 4. Doubly returning $\zeta$ with one switch

If $\zeta$ is doubly returning, $f^{p^{ \pm}}\left(\zeta^{ \pm}\right)=\zeta^{\mp}$ and $f^{p^{\mp}}\left(\zeta^{\mp}\right)=\zeta^{ \pm}$, then we say that $\zeta$ is doubly returning with two switches.

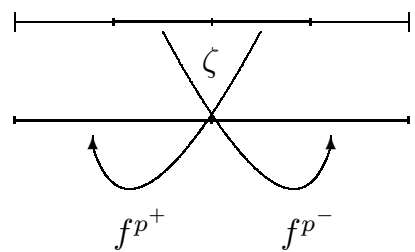

FiguRE 5. Doubly returning $\zeta$ with two switches 
In what follows consider that

$$
U_{n}^{ \pm}=U_{n} \cap f^{-\ell}\left(Y_{i^{ \pm}}\right) .
$$

The main goals of this section are to compute the EI and also the limit for the REPP at non-simple points as defined above. In the case of aperiodic non-simple points, the analysis is very similar to the one held for non-periodic points, in the previous sections, and we get an EI equal to 1 and the convergence of the REPP to the standard Poisson process. In the case of singly returning and doubly returning points, we have periodicity and consequently clustering. This means that the analysis should follow the footsteps of [12,13] with the necessary adjustments. For completeness we include a brief review of the results needed in Appendix $\mathrm{A}$ and in particular the formulas (B.1) and (B.2) in Appendix B, that we will use to compute the EI and the multiplicity distribution of the limiting compound Poisson process.

Proposition 3.4. Suppose that $(Y, f, \psi)$ is a topologically mixing Rychlik system with finitely many branches, $\psi$ is Hölder continuous on each $\bar{Y}_{i}$, and $\mu=\mu_{\psi}$ is the corresponding equilibrium state. Let $X_{0}, X_{1}, \ldots$ be given by (2.6), where $\varphi$ achieves a global maximum at some point $\zeta \in Y \backslash \Lambda$. Let $u_{n}$ be such that (2.10) holds and $U_{n}$ be defined as in (2.12). We assume that $\mu\left(U_{n}^{ \pm}\right) \sim \alpha^{ \pm} \mu\left(U_{n}\right)$, where $0<\alpha^{-}, \alpha^{+}<1$ and $\alpha^{-}+\alpha^{+}=1$. Then we have an $E V L$ for $M_{n}$ and

(1) if $\zeta$ is an aperiodic non-simple point, then the EVL is such that $\bar{H}(\tau)=\mathrm{e}^{-\tau}$;

(2) if $\zeta$ is a non-simple, repelling singly returning point, then the $E V L$ is such that $\bar{H}(\tau)=\mathrm{e}^{-\vartheta \tau}$, where the EI is given by $\vartheta=1-\alpha^{ \pm} \mathrm{e}^{S_{p^{ \pm}} \psi\left(\zeta^{ \pm}\right)}$;

(3) if $\zeta$ is a non-simple, repelling doubly returning point, then the EVL is such that $\bar{H}(\tau)=\mathrm{e}^{-\vartheta \tau}$, where the EI is given by $\vartheta=1-\alpha^{+} \mathrm{e}^{S_{p^{+}} \psi\left(\zeta^{+}\right)}-$ $\alpha^{-} \mathrm{e}^{S_{p^{-}} \psi\left(\zeta^{-}\right)}$, when $\zeta$ has no switches; $\vartheta=1-\alpha^{ \pm}\left(\mathrm{e}^{S_{p^{+}} \psi\left(\zeta^{+}\right)}+\mathrm{e}^{S_{p^{-}} \psi\left(\zeta^{-}\right)}\right)$, when $\zeta$ has one switch; $\vartheta=1-\alpha^{-} \mathrm{e}^{S_{p^{+}} \psi\left(\zeta^{+}\right)}-\alpha^{+} \mathrm{e}^{S_{p^{-}} \psi\left(\zeta^{-}\right)}$, when $\zeta$ has two switches.

Remark 3.3. We remark that, in the particular case when $\mu_{\psi}$ is absolutely continuous with respect to the Lebesgue measure and the invariant density is continuous at the points $\zeta$ considered in the proposition above, the formulas for the EI can be seen as special cases of the formula in [20, Remark 8].

Proof. If $\zeta$ is an aperiodic non-simple point, then we just have to mimic the argument for non-periodic points in the previous sections. The proof of $D_{2}\left(u_{n}\right)$ is done exactly as before. Using decay of correlations against $L^{1}$, stated in (3.2), the proof that $D^{\prime}\left(u_{n}\right)$ holds for these points follows the same footsteps except for the adjustments in order to consider the two possible evolutions corresponding to the "orbits" of $\zeta^{+}$and $\zeta^{-}$. For example, to prove that $R\left(U_{n}\right) \rightarrow \infty$, as $n \rightarrow \infty$, in the argument of Lemma 3.1 we would define

$$
\epsilon=\min \left\{\min _{k=1, \ldots, j} \operatorname{dist}\left(f^{k}\left(\zeta^{+}\right), \zeta\right), \min _{k=1, \ldots, j} \operatorname{dist}\left(f^{k}\left(\zeta^{-}\right), \zeta\right)\right\},
$$

and proceed as before.

When $\zeta$ is a non-simple (singly or doubly) returning point, we just need to adjust the definition (A.1) of $Q_{p}\left(u_{n}\right)$ to cope with the two possibly different evolutions of $\zeta^{+}$and $\zeta^{-}$. Everything else, namely the proofs of conditions $D^{p}\left(u_{n}\right)$ and $D_{p}^{\prime}\left(u_{n}\right)$, follows from decay of correlations against $L^{1}$, stated in (3.2), exactly along the same 
lines as in the proof of [13, Theorem 2]. Hence, essentially, for each different case we have to define coherently $Q_{p}\left(u_{n}\right)$ and compute the EI using formula (B.1).

Assume first that $\zeta$ is a singly returning (eventually aperiodic or not) non-simple point. Without loss of generality (w.l.o.g.), we also assume that there exists $p$ such that $f^{p}\left(\zeta^{+}\right)=\zeta^{+}$. In this case, we should define $Q_{p}\left(u_{n}\right)=U_{n}^{-} \cup\left(U_{n}^{+} \backslash f^{-p}\left(U_{n}\right)\right)$, as seen in Figure 6.

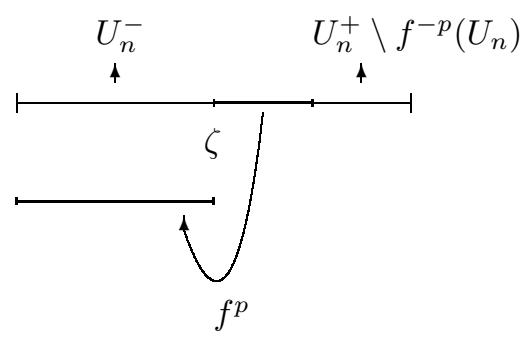

FiguRe 6. $Q_{p}\left(u_{n}\right)=U_{n}^{-} \cup\left(U_{n}^{+} \backslash f^{-p}\left(U_{n}\right)\right)$

We can now compute the EI:

$$
\begin{aligned}
\vartheta & =\lim _{n \rightarrow \infty} \frac{\mu\left(Q_{p}\left(u_{n}\right)\right)}{\mu\left(U_{n}\right)}=\lim _{n \rightarrow \infty} \frac{\mu\left(U_{n}^{-}\right)+\left(1-\mathrm{e}^{S_{p} \psi\left(\zeta^{+}\right)}\right) \mu\left(U_{n}^{+}\right)}{\mu\left(U_{n}\right)} \\
& =\lim _{n \rightarrow \infty} \frac{\alpha^{-} \mu\left(U_{n}\right)+\alpha^{+}\left(1-\mathrm{e}^{S_{p} \psi\left(\zeta^{+}\right)}\right) \mu\left(U_{n}\right)}{\mu\left(U_{n}\right)}=1-\alpha^{+} \mathrm{e}^{S_{p} \psi\left(\zeta^{+}\right)} .
\end{aligned}
$$

Let $\zeta$ be a non-simple, repelling doubly returning point and $p^{-}, p^{+}$be such that $f^{p^{-}}\left(\zeta^{-}\right)=\zeta$ and $f^{p^{+}}\left(\zeta^{+}\right)=\zeta$. For definiteness, we assume w.l.o.g. that $p^{-}<p^{+}$.

First we consider the case where no switching occurs. In this case, we have two different "periods", hence we should define $Q_{p^{-}, p^{+}}\left(u_{n}\right)=\left(U_{n}^{-} \backslash f^{-p^{-}}\left(U_{n}^{-}\right)\right) \cup$ $\left(U_{n}^{+} \backslash f^{-p^{+}}\left(U_{n}^{+}\right)\right)$.

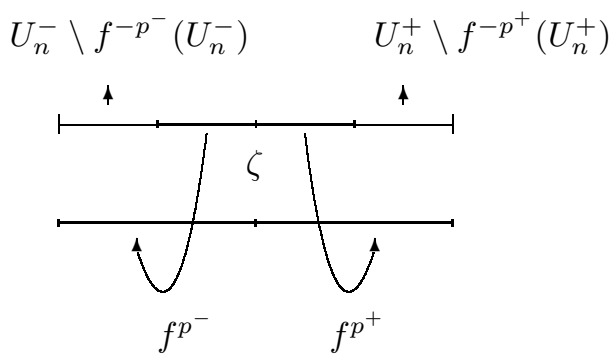

Figure 7. $Q_{p^{-}, p^{+}}\left(u_{n}\right)=\left(U_{n}^{-} \backslash f^{-p^{-}}\left(U_{n}^{-}\right)\right) \cup\left(U_{n}^{+} \backslash f^{-p^{+}}\left(U_{n}^{+}\right)\right)$

It follows that

$$
\begin{aligned}
\vartheta & =\lim _{n \rightarrow \infty} \frac{\alpha^{-}\left(1-\mathrm{e}^{S_{p} \psi\left(\zeta^{-}\right)}\right) \mu\left(U_{n}\right)+\alpha^{+}\left(1-\mathrm{e}^{S_{p} \psi\left(\zeta^{+}\right)}\right) \mu\left(U_{n}\right)}{\mu\left(U_{n}\right)} \\
& =1-\alpha^{-} \mathrm{e}^{S_{p} \psi\left(\zeta^{-}\right)}-\alpha^{+} \mathrm{e}^{S_{p} \psi\left(\zeta^{+}\right)}
\end{aligned}
$$


Next, we consider the case with one switch. In this case, we also have two different "periods", and for definiteness we assume w.l.o.g. that $f^{p^{-}}\left(\zeta^{-}\right)=\zeta^{-}$and $f^{p^{+}}\left(\zeta^{+}\right)=\zeta^{-}$. Then we define $Q_{p^{-}, p^{+}}\left(u_{n}\right)=\left(U_{n}^{-} \backslash f^{-p^{-}}\left(U_{n}^{-}\right)\right) \cup\left(U_{n}^{+} \backslash f^{-p^{+}}\left(U_{n}^{-}\right)\right)$.

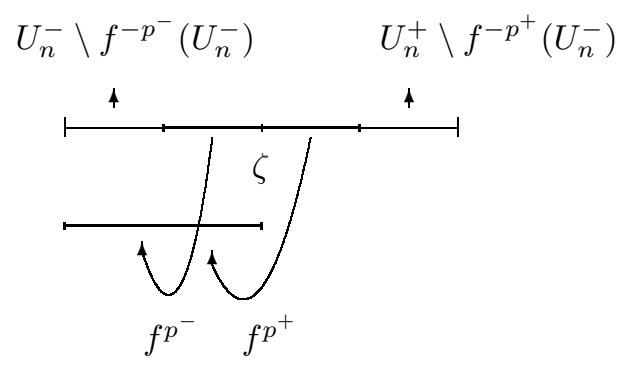

FIGURE 8. $Q_{p^{-}, p^{+}}\left(u_{n}\right)=\left(U_{n}^{-} \backslash f^{-p^{-}}\left(U_{n}^{-}\right)\right) \cup\left(U_{n}^{+} \backslash f^{-p^{+}}\left(U_{n}^{-}\right)\right)$

It follows that

$$
\begin{aligned}
\vartheta & =\lim _{n \rightarrow \infty} \frac{\alpha^{-}\left(1-\mathrm{e}^{S_{p} \psi\left(\zeta^{-}\right)}\right) \mu\left(U_{n}\right)+\alpha^{-}\left(1-\mathrm{e}^{S_{p} \psi\left(\zeta^{+}\right)}\right) \mu\left(U_{n}\right)}{\mu\left(U_{n}\right)} \\
& =1-\alpha^{-} \mathrm{e}^{S_{p} \psi\left(\zeta^{-}\right)}-\alpha^{-} \mathrm{e}^{S_{p} \psi\left(\zeta^{+}\right)}
\end{aligned}
$$

Finally, we consider the case with two switches. In this case, we also have two different "periods" and we should define $Q_{p^{-}, p^{+}}\left(u_{n}\right)=\left(U_{n}^{-} \backslash f^{-p^{-}}\left(U_{n}^{+}\right)\right) \cup$ $\left(U_{n}^{+} \backslash f^{-p^{+}}\left(U_{n}^{-}\right)\right)$.

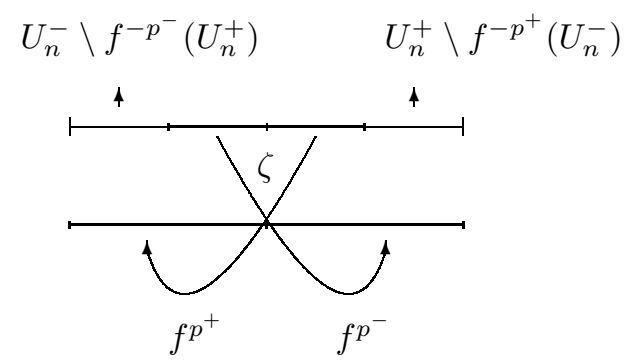

FIGURE 9. $Q_{p^{-}, p^{+}}\left(u_{n}\right)=\left(U_{n}^{-} \backslash f^{-p^{-}}\left(U_{n}^{+}\right)\right) \cup\left(U_{n}^{+} \backslash f^{-p^{+}}\left(U_{n}^{-}\right)\right)$

It follows that

$$
\begin{aligned}
\vartheta & =\lim _{n \rightarrow \infty} \frac{\alpha^{+}\left(1-\mathrm{e}^{S_{p} \psi\left(\zeta^{-}\right)}\right) \mu\left(U_{n}\right)+\alpha^{-}\left(1-\mathrm{e}^{S_{p} \psi\left(\zeta^{+}\right)}\right) \mu\left(U_{n}\right)}{\mu\left(U_{n}\right)} \\
& =1-\alpha^{-} \mathrm{e}^{S_{p} \psi\left(\zeta^{+}\right)}-\alpha^{+} \mathrm{e}^{S_{p} \psi\left(\zeta^{-}\right)}
\end{aligned}
$$

The next result gives the convergence of the REPP at non-simple points. Note that, contrary to the usual geometric distribution obtained, for example, in 6 , 13, 16], here the multiplicity distribution is quite different. In fact, for eventually aperiodic returning points, for example, we have that $\pi(\kappa)=0$ for all $\kappa \geq 3$. 
Proposition 3.5. Let $a^{ \pm}:=\mathrm{e}^{S_{p^{ \pm}} \psi\left(\zeta^{ \pm}\right)}$. Under the same assumptions as Proposition 3.4, we have:

(1) if $\zeta$ is an aperiodic non-simple point, then the REPP converges to a standard Poisson process of intensity 1 ;

(2) if $\zeta$ is a non-simple, singly returning point

(a) not eventually aperiodic, then the REPP converges to a compound Poisson process of intensity $\vartheta$, given in Proposition [3.4, and multiplicity distribution defined by:

$$
\pi(1)=\frac{\vartheta-(1-\vartheta)\left(1-a^{ \pm}\right)}{\vartheta}, \quad \pi(\kappa)=\frac{\alpha^{ \pm}\left(1-a^{ \pm}\right)^{2}\left(a^{ \pm}\right)^{-(\kappa-1)}}{\vartheta}, \quad \kappa \geq 2 .
$$

(b) eventually aperiodic, then the REPP converges to a compound Poisson process of intensity $\vartheta$, given in Proposition [3.4, and multiplicity distribution defined by:

$$
\pi(1)=\frac{2 \vartheta-1}{\vartheta}, \quad \pi(2)=\frac{1-\vartheta}{\vartheta}, \quad \pi(\kappa)=0, \quad \kappa \geq 3 .
$$

(3) if $\zeta$ is a non-simple, repelling doubly returning point

(a) with no switches, then the REPP converges to a compound Poisson process of intensity $\vartheta$, given in Proposition 3.4, and multiplicity distribution defined by:

$$
\begin{aligned}
& \pi(1)=\frac{2 \vartheta-1+\alpha^{-}\left(a^{-}\right)^{2}+\alpha^{+}\left(a^{+}\right)^{2}}{\vartheta}, \\
& \pi(\kappa)=\frac{\alpha^{-}\left(1-a^{-}\right)^{2}\left(a^{-}\right)^{-(\kappa-1)}+\alpha^{-}\left(1-a^{+}\right)^{2}\left(a^{+}\right)^{-(\kappa-1)}}{\vartheta}, \kappa \geq 2 .
\end{aligned}
$$

(b) with one switch, then the REPP converges to a compound Poisson process of intensity $\vartheta$, given in Proposition 3.4, and multiplicity distribution defined by:

$$
\pi(1)=\frac{2 \vartheta-1+a^{ \pm}(1-\vartheta)}{\vartheta}, \pi(\kappa)=\frac{(1-\vartheta)\left(a^{ \pm}\right)^{\kappa-2}\left(1-a^{ \pm}\right)^{2}}{\vartheta}, \kappa \geq 2 .
$$

(c) with two switches, then the REPP converges to a compound Poisson process of intensity $\vartheta$, given in Proposition 3.4, and multiplicity distribution defined by:

$$
\begin{aligned}
& \pi(1)=\frac{1-2(1-\vartheta)+a^{-} a^{+}}{\vartheta}, \quad \pi(2 j)=\frac{\left(a^{-} a^{+}\right)^{j-1}\left((1-\vartheta)\left(1+a^{-} a^{+}\right)-2 a^{-} a^{+}\right)}{\vartheta}, \\
& \pi(2 j+1)=\frac{\left(a^{-} a^{+}\right)^{j}\left(1-2(1-\vartheta)+a^{-} a^{+}\right)}{\vartheta}, j \geq 1 .
\end{aligned}
$$

Proof. When $\zeta$ is an aperiodic non-simple point, then as we have seen in Proposition 3.4. condition $D^{\prime}\left(u_{n}\right)$ holds. Clearly, $D_{3}\left(u_{n}\right)$ follows from decay of correlations and, by [10, Theorem 5], we easily conclude that the REPP converges to the standard Poisson process of intensity 1.

When $\zeta$ is a non-simple (singly or doubly) returning point, we just need to adjust the definition of the sets $U^{(\kappa)}$ given in (A.3), which ultimately affects the sets $Q_{p}^{\kappa}(u)$, given in (A.4), in order to cope with the two possibly different evolutions of $\zeta^{+}$and $\zeta^{-}$. Everything else, namely the proofs of conditions $D^{p}\left(u_{n}\right)^{*}$ and $D_{p}^{\prime}\left(u_{n}\right)^{*}$, follows from decay of correlations against $L^{1}$, stated in (3.2), exactly along the same lines as in the proof of [13. Theorem 2]. Hence, essentially, for each different case we have to define coherently the sets $U^{(\kappa)}$ and compute the multiplicity distribution using formula (B.2).

In all cases, $U^{(0)}=U_{n}=U_{n}^{-} \cup U_{n}^{+}$. 
Let $\zeta$ be a singly returning non-simple point which is not eventually aperiodic. We assume w.l.o.g. that there exists $p$ such that $f^{p}\left(\zeta^{+}\right)=\zeta^{+}$and $f^{j}\left(\zeta^{-}\right) \neq \zeta$, for all $j \in \mathbb{N}$. For every $\kappa \in \mathbb{N}$, we define

$$
U^{(\kappa)}:=\left(\bigcap_{i=0}^{\kappa} f^{-i p}\left(U_{n}^{+}\right)\right) .
$$

Using (A.4), we can now easily define $Q^{\kappa}:=U^{(\kappa)} \backslash U^{(\kappa+1)}$, for all $\kappa \geq 0$. We have $\mathbb{P}\left(Q^{0}\right) \sim \mathbb{P}\left(U_{n}\right)-a^{+} \mathbb{P}\left(U_{n}^{+}\right) \sim \mathbb{P}\left(U_{n}\right)\left(1-\alpha^{+} a^{+}\right)$. The same computations would lead us to $\mathbb{P}\left(Q^{\kappa}\right) \sim \mathbb{P}\left(U_{n}\right)\left(\alpha^{+}\left(1-a^{+}\right)\left(a^{+}\right)^{\kappa}\right)$. Using formula (B.2), it follows that

$$
\begin{aligned}
\pi(1) & =\lim _{n \rightarrow \infty} \frac{\mathbb{P}\left(Q^{0}\right)-\mathbb{P}\left(Q^{1}\right)}{\mathbb{P}\left(Q^{0}\right)}=\frac{\left(1-\alpha^{+} a^{+}\right)-\left(\alpha^{+}\left(1-a^{+}\right) a^{+}\right)}{\left(1-\alpha^{+} a^{+}\right)}=\frac{\vartheta-(1-\vartheta)\left(1-a^{ \pm}\right)}{\vartheta}, \\
\pi(\kappa) & =\lim _{n \rightarrow \infty} \frac{\mathbb{P}\left(Q^{\kappa-1}\right)-\mathbb{P}\left(Q^{\kappa}\right)}{\mathbb{P}\left(Q^{0}\right)}=\frac{\left(\alpha^{+}\left(1-a^{+}\right)\left(a^{+}\right)^{\kappa-1}\right)-\left(\alpha^{+}\left(1-a^{+}\right)\left(a^{+}\right)^{\kappa}\right)}{\left(1-\alpha^{+} a^{+}\right)} \\
& =\frac{\alpha^{+}\left(1-a^{+}\right)^{2}\left(a^{+}\right)^{\kappa-1}}{\vartheta} .
\end{aligned}
$$

Let $\zeta$ be a singly returning non-simple point which is eventually aperiodic. We assume w.l.o.g. that there exists $p$ such that $f^{p}\left(\zeta^{+}\right)=\zeta^{-}$and $f^{j}\left(\zeta^{-}\right) \neq \zeta$, for all $j \in \mathbb{N}$. For every $\kappa \in \mathbb{N}$, we define

$$
U^{(1)}:=\left(U_{n}^{+} \cap f^{-i p}\left(U_{n}^{-}\right)\right), \quad U^{(\kappa)}:=\emptyset, \quad \kappa \geq 2 .
$$

Note that $Q^{0}=U_{n} \backslash U^{(1)}, Q^{1}=U^{(1)}$ and $Q^{\kappa}=\emptyset$, for all $\kappa \geq 2$. We have $\mathbb{P}\left(Q^{0}\right) \sim \mathbb{P}\left(U_{n}\right)-a^{+} \mathbb{P}\left(U_{n}^{-}\right) \sim \mathbb{P}\left(U_{n}\right)\left(1-\alpha^{-} a^{+}\right), \mathbb{P}\left(Q^{1}\right) \sim \mathbb{P}\left(U_{n}\right) \alpha^{-} a^{+}, \mathbb{P}\left(Q^{\kappa}\right)=0$, for all $\kappa \geq 2$. Using formula (B.2), it follows that

$$
\begin{array}{r}
\pi(1)=\lim _{n \rightarrow \infty} \frac{\mathbb{P}\left(Q^{0}\right)-\mathbb{P}\left(Q^{1}\right)}{\mathbb{P}\left(Q^{0}\right)} \\
=\frac{\left(1-\alpha^{-} a^{+}\right)-\left(\alpha^{-} a^{+}\right)}{\left(1-\alpha^{-} a^{+}\right)}=\frac{2 \vartheta-1}{\vartheta}, \\
\pi(2)=\lim _{n \rightarrow \infty} \frac{\mathbb{P}\left(Q^{1}\right)-\mathbb{P}\left(Q^{2}\right)}{\mathbb{P}\left(Q^{0}\right)}=\frac{\alpha^{-} a^{+}}{\left(1-\alpha^{-} a^{+}\right)} \\
=\frac{1-\vartheta}{\vartheta}, \quad \pi(\kappa)=0, \quad k \geq 3 .
\end{array}
$$

Let $\zeta$ be a doubly returning non-simple point with no switches. Let $p^{-}, p^{+}$be such that $f^{p^{-}}\left(\zeta^{-}\right)=\zeta^{-}$and $f^{p^{+}}\left(\zeta^{+}\right)=\zeta^{+}$. For every $\kappa \in \mathbb{N}$, we define

$$
U^{(\kappa)}:=\left(\bigcap_{i=0}^{\kappa} f^{-i p^{-}}\left(U_{n}^{-}\right)\right) \cup\left(\bigcap_{i=0}^{\kappa} f^{-i p^{+}}\left(U_{n}^{+}\right)\right) .
$$

Note that using (A.4), we can now easily define $Q^{\kappa}:=U^{(\kappa)} \backslash U^{(\kappa+1)}$, for all $\kappa \geq 0$. We have $\mathbb{P}\left(Q^{0}\right) \sim \mathbb{P}\left(U_{n}\right)-a^{-} \mathbb{P}\left(U_{n}^{-}\right)-a^{+} \mathbb{P}\left(U_{n}^{+}\right) \sim \mathbb{P}\left(U_{n}\right)\left(1-\alpha^{-} a^{-}-\alpha^{+} a^{+}\right)$. The same computations would lead us to

$$
\mathbb{P}\left(Q^{\kappa}\right) \sim \mathbb{P}\left(U_{n}\right)\left(\alpha^{-}\left(1-a^{-}\right)\left(a^{-}\right)^{\kappa}+\alpha^{+}\left(1-a^{+}\right)\left(a^{+}\right)^{\kappa}\right) .
$$

Using formula (B.2), it follows that

$$
\begin{gathered}
\pi(1)=\lim _{n \rightarrow \infty} \frac{\mathbb{P}\left(Q^{0}\right)-\mathbb{P}\left(Q^{1}\right)}{\mathbb{P}\left(Q^{0}\right)}=\frac{\left(1-\alpha^{-} a^{-}-\alpha^{+} a^{+}\right)-\left(\alpha^{-}\left(1-a^{-}\right) a^{-}+\alpha^{+}\left(1-a^{+}\right) a^{+}\right)}{\left(1-\alpha^{-} a^{-}-\alpha^{+} a^{+}\right)} \\
=\frac{2 \vartheta-1+\alpha^{-}\left(a^{-}\right)^{2}+\alpha^{+}\left(a^{+}\right)^{2}}{\vartheta}, \\
\pi(\kappa)=\lim _{n \rightarrow \infty} \frac{\mathbb{P}\left(Q^{\kappa-1}\right)-\mathbb{P}\left(Q^{\kappa}\right)}{\mathbb{P}\left(Q^{0}\right)}=\frac{\alpha^{-}\left(1-a^{-}\right)^{2}\left(a^{-}\right)^{\kappa-1}+\alpha^{+}\left(1-a^{+}\right)^{2}\left(a^{+}\right)^{\kappa-1}}{\vartheta} .
\end{gathered}
$$


Let $\zeta$ be a doubly returning non-simple point with one switch. We assume w.l.o.g. that there exist $p^{-}, p^{+}$such that $f^{p^{-}}\left(\zeta^{-}\right)=\zeta^{-}$and $f^{p^{+}}\left(\zeta^{+}\right)=\zeta^{-}$. For every $\kappa \in \mathbb{N}$, we define

$$
U^{(\kappa)}:=\left(\bigcap_{i=0}^{\kappa} f^{-i p^{-}}\left(U_{n}^{-}\right)\right) \cup\left(U_{n}^{+} \cap f^{-p^{+}}\left(U_{n}^{-}\right) \cap \bigcap_{i=0}^{\kappa} f^{-p^{+}-i p^{-}}\left(U_{n}^{-}\right)\right) .
$$

Using A.4), we can now easily define $Q^{\kappa}:=U^{(\kappa)} \backslash U^{(\kappa+1)}$, for all $\kappa \geq 0$. We have $\mathbb{P}\left(Q^{0}\right) \sim \mathbb{P}\left(U_{n}\right)-a^{-} \mathbb{P}\left(U_{n}^{-}\right)-a^{+} \mathbb{P}\left(U_{n}^{-}\right) \sim \mathbb{P}\left(U_{n}\right)\left(1-\alpha^{-} a^{-}-\alpha^{-} a^{+}\right)$. The same computations would lead us to

$$
\mathbb{P}\left(Q^{\kappa}\right) \sim \mathbb{P}\left(U_{n}\right)\left(\alpha^{-}\left(1-a^{-}\right)\left(a^{-}\right)^{\kappa}+\alpha^{-}\left(1-a^{-}\right) a^{+}\left(a^{-}\right)^{\kappa-1}\right) .
$$

Using formula (B.2), it follows that

$$
\begin{aligned}
\pi(1) & =\lim _{n \rightarrow \infty} \frac{\mathbb{P}\left(Q^{0}\right)-\mathbb{P}\left(Q^{1}\right)}{\mathbb{P}\left(Q^{0}\right)}=\frac{\left(1-\alpha^{-}\left(a^{-}+a^{+}\right)\right)-\left(\alpha^{-}\left(1-a^{-}\right) a^{-}+\alpha^{-}\left(1-a^{-}\right) a^{+}\right)}{\left(1-\alpha^{-} a^{-}-\alpha^{-} a^{+}\right)} \\
& =\frac{2 \vartheta-1+a^{-}(1-\vartheta)}{\vartheta}, \\
\pi(\kappa) & =\lim _{n \rightarrow \infty} \frac{\mathbb{P}\left(Q^{\kappa-1}\right)-\mathbb{P}\left(Q^{\kappa}\right)}{\mathbb{P}\left(Q^{0}\right)}=\frac{\alpha^{-}\left(1-a^{-}\right)^{2}\left(a^{-}\right)^{\kappa-1}+\alpha^{-}\left(1-a^{-}\right)^{2} a^{+}\left(a^{-}\right)^{\kappa-2}}{\vartheta} \\
& =\frac{(1-\vartheta)\left(a^{-}\right)^{\kappa-2}\left(1-a^{-}\right)^{2}}{\vartheta} .
\end{aligned}
$$

Let $\zeta$ be a doubly returning non-simple point with two switches. We assume that there exist $p^{-}, p^{+}$such that $f^{p^{-}}\left(\zeta^{-}\right)=\zeta^{+}$and $f^{p^{+}}\left(\zeta^{+}\right)=\zeta^{-}$. For every $j \in \mathbb{N}_{0}$, we define

$$
\begin{aligned}
U^{(2 j+1)} & :=\left(U_{n}^{-} \cap \bigcap_{i=1}^{j+1} f^{-i p^{-}-(i-1) p^{+}}\left(U_{n}^{+}\right) \cap \bigcap_{i=1}^{j} f^{-i p^{-}-i p^{+}}\left(U_{n}^{-}\right)\right) \\
& \cup\left(U_{n}^{+} \cap \bigcap_{i=1}^{j+1} f^{-i p^{+}-(i-1) p^{-}}\left(U_{n}^{-}\right) \cap \bigcap_{i=1}^{j} f^{-i p^{+}-i p^{-}}\left(U_{n}^{+}\right)\right), \\
U^{(2 j)} & :=\left(U_{n}^{-} \cap \bigcap_{i=1}^{j} f^{-i p^{-}-(i-1) p^{+}}\left(U_{n}^{+}\right) \cap \bigcap_{i=1}^{j} f^{-i p^{-}-i p^{+}}\left(U_{n}^{-}\right)\right) \\
& \cup\left(U_{n}^{+} \cap \bigcap_{i=1}^{j} f^{-i p^{+}-(i-1) p^{-}}\left(U_{n}^{-}\right) \cap \bigcap_{i=1}^{j} f^{-i p^{+}-i p^{-}}\left(U_{n}^{+}\right)\right) .
\end{aligned}
$$

Using (A.4), we can now easily define $Q^{\kappa}:=U^{(\kappa)} \backslash U^{(\kappa+1)}$, for all $\kappa \geq 0$. We have $\mathbb{P}\left(Q^{0}\right) \sim \mathbb{P}\left(U_{n}\right)-a^{-} \mathbb{P}\left(U_{n}^{+}\right)-a^{+} \mathbb{P}\left(U_{n}^{-}\right) \sim \mathbb{P}\left(U_{n}\right)\left(1-\alpha^{+} a^{-}-\alpha^{-} a^{+}\right)$. The same computations would lead us to $\mathbb{P}\left(Q^{2 j}\right) \sim \mathbb{P}\left(U_{n}\right)\left(1-\alpha^{+} a^{-}-\alpha^{-} a^{+}\right)\left(a^{-} a^{+}\right)^{j}$ and $\mathbb{P}\left(Q^{2 j}\right) \sim \mathbb{P}\left(U_{n}\right)\left(\alpha^{+} a^{-}+\alpha^{-} a^{+}-a^{-} a^{+}\right)\left(a^{-} a^{+}\right)^{j}$. Using formula (B.2), it follows that, for every $j \in \mathbb{N}$,

$$
\begin{aligned}
& \pi(1)=\lim _{n \rightarrow \infty} \frac{\mathbb{P}\left(Q^{0}\right)-\mathbb{P}\left(Q^{1}\right)}{\mathbb{P}\left(Q^{0}\right)}=\frac{\left(1-\alpha^{+} a^{-}-\alpha^{-} a^{+}\right)-\left(\alpha^{+} a^{-}+\alpha^{-} a^{+}-a^{-} a^{+}\right)}{\left(1-\alpha^{+} a^{-}-\alpha^{-} a^{+}\right)}=\frac{1-2(1-\vartheta)+a^{-} a^{+}}{\vartheta}, \\
& \pi(2 j)=\lim _{n \rightarrow \infty} \frac{\mathbb{P}\left(Q^{2 j-1}\right)-\mathbb{P}\left(Q^{2 j}\right)}{\mathbb{P}\left(Q^{0}\right)}=\frac{\left(a^{-} a^{+}\right)^{j-1}\left(\alpha^{+} a^{-}\left(1+a^{-} a^{+}\right)+\alpha^{-} a^{+}\left(1+a^{-} a^{+}\right)-2 a^{-} a^{+}\right)}{\vartheta}, \\
& \pi(2 j+1)=\lim _{n \rightarrow \infty} \frac{\mathbb{P}\left(Q^{2 j}\right)-\mathbb{P}\left(Q^{2 j+1}\right)}{\mathbb{P}\left(Q^{0}\right)}=\frac{\left(a^{-} a^{+}\right)^{j}\left(1-2 \alpha^{+} a^{-}-2 \alpha^{-} a^{+}+a^{-} a^{+}\right)}{\vartheta} .
\end{aligned}
$$




\section{EXTREMES FOR RANDOM DYNAMiCS}

In this section we will start with the proof of Theorem $\mathrm{D}$ which states that the dichotomy observed in Section 3 vanishes when we add absolutely continuous noise (w.r.t. Lebesgue) and for every chosen point in the phase space we have a standard exponential distribution for the EVL and HTS/RTS weak limits. We will also certify that the REPP converges to a Poisson Process with intensity 1 . Next, we will give some examples of random dynamical systems for which we can prove the existence of EVLs and HTS/RTS as well as the convergence of REPP.

In what follows, we denote the diameter of set $A \subset \mathcal{M}$ by

$$
|A|:=\sup \{\operatorname{dist}(x, y): x, y \in A\},
$$

and for any $x \in \mathcal{M}$ we define the translation of $A$ by $x$ as the set $A+x:=\{a+x$ : $a \in A\}$.

\subsection{Laws of rare events for random dynamics.}

Proof of Theorem D, Firstly, we want to show that, as in the deterministic case, the condition $D_{2}\left(u_{n}\right)$ can be deduced from the decay of correlations.

From our assumption the random dynamical system has (annealed) decay of correlations, i.e., there exists a Banach space $\mathcal{C}$ of real valued functions such that for all $\phi \in \mathcal{C}$ and $\psi \in L^{1}\left(\mu_{\varepsilon}\right)$,

$$
\left|\int \phi\left(\mathcal{U}_{\varepsilon}^{t} \psi\right)(x) \mathrm{d} \mu_{\varepsilon}-\int \phi \mathrm{d} \mu_{\varepsilon} \int \psi \mathrm{d} \mu_{\varepsilon}\right| \leq C\|\phi\|_{\mathcal{C}}\|\psi\|_{L^{1}\left(\mu_{\varepsilon}\right)} t^{-2},
$$

where $C>0$ is a constant independent of both $\varphi$ and $\psi$.

In proving $D_{2}\left(U_{n}\right)$, the main point is to choose the right observable. We take

$$
\begin{gathered}
\phi(x)=\mathbf{1}_{\left\{X_{0}>u_{n}\right\}}=\mathbf{1}_{\left\{\varphi(x)>u_{n}\right\}}, \\
\psi(x)=\int \mathbf{1}_{\left\{\varphi(x), \varphi \circ f_{\tilde{\omega}_{1}}(x), \ldots, \varphi \circ f_{\underline{\underline{\omega}}}^{\ell-1}(x) \leq u_{n}\right\}} \mathrm{d} \theta_{\varepsilon}^{\ell-1}(\underline{\tilde{\tilde{\omega}}}) .
\end{gathered}
$$

Substituting $\psi$ in the random evolution operator, we get

$$
\left(\mathcal{U}_{\varepsilon}^{t} \psi\right)(x)=\iint \mathbf{1}_{\left\{\varphi \circ f_{\underline{\omega}}^{t}(x), \ldots, \varphi \circ f_{\underline{\tilde{\omega}}}^{\ell-1} \circ f_{\underline{\omega}}^{t}(x) \leq u_{n}\right\}} \mathrm{d} \theta_{\varepsilon}^{\ell-1}(\underline{\tilde{\omega}}) \mathrm{d} \theta_{\varepsilon}^{t}(\underline{\omega}) .
$$

Since all $\omega_{i}$ 's and $\tilde{\omega}_{j}$ 's are chosen in an i.i.d. structure, we can rename the random iterates, i.e., we lose no information in writing

$$
\left(\mathcal{U}_{\varepsilon}^{t} \psi\right)(x)=\int \mathbf{1}_{\left\{\varphi \circ f_{\underline{\omega}}^{t}(x), \ldots, \varphi \circ f_{\underline{\omega}}^{t+\ell-1}(x) \leq u_{n}\right\}} \mathrm{d} \theta_{\varepsilon}^{\mathbb{N}}(\underline{\omega}) .
$$

Therefore, we get

$$
\begin{aligned}
& \int \phi(x)\left(\mathcal{U}_{\varepsilon}^{t} \psi\right)(x) \mathrm{d} \mu_{\varepsilon} \\
& \quad=\int \mu_{\varepsilon}\left(\varphi(x)>u_{n}, \varphi \circ f_{\underline{\omega}}^{t}(x) \leq u_{n}, \ldots, \varphi \circ f_{\underline{\omega}}^{t+\ell-1}(x) \leq u_{n}\right) \mathrm{d} \theta_{\varepsilon}^{\mathbb{N}}(\underline{\omega}) .
\end{aligned}
$$


On the other hand,

$$
\begin{aligned}
\int \phi(x) \mathrm{d} \mu_{\varepsilon} & =\mu_{\varepsilon}\left(X_{0}(x)>u_{n}\right)=\int \mu_{\varepsilon}\left(X_{0}(x)>u_{n}\right) \mathrm{d} \theta_{\varepsilon}^{\mathbb{N}}(\underline{\omega}), \\
\int \psi(x) \mathrm{d} \mu_{\varepsilon} & =\int\left(\int \mathbf{1}_{\left\{\varphi(x), \varphi \circ f_{\omega_{1}}(x), \ldots, \varphi \circ f_{\underline{\omega}}^{\ell-1}(x) \leq u_{n}\right\}} \mathrm{d} \mu_{\varepsilon}\right) \mathrm{d} \theta_{\varepsilon}^{\mathbb{N}(\underline{\omega})} \\
& =\int \mu_{\varepsilon}\left(\varphi(x) \leq u_{n}, \varphi \circ f_{\omega_{1}}(x) \leq u_{n}, \ldots, \varphi \circ f_{\underline{\omega}}^{\ell-1}(x) \leq u_{n}\right) \mathrm{d} \theta_{\varepsilon}^{\mathbb{N}}(\underline{\omega}) .
\end{aligned}
$$

Now, the decay of correlations can be written as

$$
\begin{aligned}
\mid \int \mu_{\varepsilon} & \left(X_{0}(x)>u_{n}, \varphi \circ f_{\underline{\omega}}^{t}(x) \leq u_{n}, \ldots, \varphi \circ f_{\underline{\omega}}^{t+\ell-1}(x) \leq u_{n}\right) \mathrm{d} \theta_{\varepsilon}^{\mathbb{N}}(\underline{\omega}) \\
& -\int \mu_{\varepsilon}\left(\varphi(x)>u_{n}\right) \mathrm{d} \theta_{\varepsilon}^{\mathbb{N}}(\underline{\omega}) \\
& \times \int \mu_{\varepsilon}\left(\varphi(x) \leq u_{n}, \varphi \circ f_{\omega_{1}}(x) \leq u_{n}, \ldots, \varphi \circ f_{\underline{\omega}}^{\ell-1}(x) \leq u_{n}\right) \mathrm{d} \theta_{\varepsilon}^{\mathbb{N}}(\underline{\omega}) \mid \\
\leq & C\|\phi\|_{\mathcal{C}}\|\psi\|_{L^{1}\left(\mu_{\varepsilon}\right)} t^{-2},
\end{aligned}
$$

which leads us to the conclusion that the condition $D_{2}\left(u_{n}\right)$ holds with

$$
\gamma(n, t)=\gamma(t)=C^{*} t^{-2}
$$

for some $C^{*}>0$ and $t_{n}=n^{\beta}$, with $1 / 2<\beta<1$.

For proving $D^{\prime}\left(u_{n}\right)$, the basic idea is to use the fact that we have decay of correlations against $L^{1}$ as in Theorem $\mathrm{A}$ and then to show that except for a small set of $\underline{\omega}$ 's, $R^{\underline{\omega}}\left(U_{n}\right)$ grows at a sufficiently fast rate. Hence, we split $\Omega$ into two parts: the $\underline{\omega}$ 's for which $R^{\underline{\omega}}\left(U_{n}\right)>\alpha_{n}$ and for which $R^{\underline{\omega}}\left(U_{n}\right) \leq \alpha_{n}$, where $\left(\alpha_{n}\right)_{n}$ is some sequence such that

$$
\alpha_{n} \rightarrow \infty \quad \text { and } \quad \alpha_{n}=o\left(\log k_{n}\right),
$$

which is designed, on one hand, to guarantee that for the $\underline{\omega}$ 's for which $R^{\underline{\omega}}\left(U_{n}\right)>$ $\alpha_{n}$, the argument using decay of correlations against $L^{1}$ is still applicable and, on the other hand, the set of the $\underline{\omega}$ 's for which $R^{\underline{\omega}}\left(U_{n}\right) \leq \alpha_{n}$ has $\theta_{\varepsilon}^{\mathbb{N}}$ small measure. To show the latter we make an estimate on the $\underline{\omega}$ 's that take the orbit of $\zeta$ too close to itself.

First, note that since $f$ is continuous (which implies that $f_{\omega}^{j}$ is also continuous for all $j \in \mathbb{N}$ ) and $\eta$ is the highest rate at which points can separate, the diameter of $f_{\underline{\omega}}^{j}\left(U_{n}\right)$ grows at most at a rate given by $\eta^{j}$, so, for any $\underline{\omega} \in \Omega$ we have $\left|f_{\underline{\omega}}^{j}\left(U_{n}\right)\right| \leq$ $\eta^{\bar{j}}\left|U_{n}\right|$. This implies that

$$
\text { if } \operatorname{dist}\left(f_{\underline{\omega}}^{j}(\zeta), \zeta\right)>2 \eta^{j}\left|U_{n}\right|>\left|U_{n}\right|+\eta^{j}\left|U_{n}\right| \text {, then } f_{\underline{\omega}}^{j}\left(U_{n}\right) \cap U_{n}=\emptyset \text {. }
$$

Note that, by equation (4.4), if for all $j=1, \ldots, \alpha_{n}$ we have $\operatorname{dist}\left(f_{\underline{\omega}}^{j}(\zeta), \zeta\right)>$ $2 \eta^{j}\left|U_{n}\right|$, then clearly $R \underline{\omega}\left(U_{n}\right)>\alpha_{n}$. Hence, we may write that $\left\{\underline{\omega}: R^{\underline{\omega}}\left(U_{n}\right) \leq\right.$ $\left.\alpha_{n}\right\} \subset \bigcup_{j=1}^{\alpha_{n}}\left\{\underline{\omega}: f_{\underline{\omega}}^{j}(\zeta) \in B_{2 \eta^{j}\left|U_{n}\right|}(\zeta)\right\}$. It follows that, there exists some $C>0$ 
such that

$$
\begin{aligned}
\theta_{\varepsilon}^{\mathbb{N}} & \left(\left\{\underline{\omega}: R^{\underline{\omega}}\left(U_{n}\right) \leq \alpha_{n}\right\}\right) \leq \sum_{j=1}^{\alpha_{n}} \int \theta_{\varepsilon}\left(\left\{\omega_{j}: f\left(f_{\underline{\omega}}^{j-1}(\zeta)\right)+\omega_{j} \in B_{2 \eta^{j}\left|U_{n}\right|}(\zeta)\right\}\right) \mathrm{d} \theta_{\varepsilon}^{\mathbb{N}} \\
& =\sum_{j=1}^{\alpha_{n}} \int \theta_{\varepsilon}\left(\left\{\omega_{j}: \omega_{j} \in B_{2 \eta^{j}\left|U_{n}\right|}(\zeta)-f\left(f_{\underline{\omega}}^{j-1}(\zeta)\right)\right\}\right) \mathrm{d} \theta_{\varepsilon}^{\mathbb{N}} \\
& =\sum_{j=1}^{\alpha_{n}} \iint_{B_{2 \eta^{j}\left|U_{n}\right|}(\zeta)-f\left(f_{\underline{\omega}}^{j-1}(\zeta)\right)} g_{\varepsilon}(x) \mathrm{dLebd} \theta_{\varepsilon}^{\mathbb{N}} \\
& \leq \sum_{j=1}^{\alpha_{n}} \overline{g_{\varepsilon}} \operatorname{Leb}\left(B_{2 \eta^{j}\left|U_{n}\right|}(\zeta)\right)=\sum_{j=1}^{\alpha_{n}} \overline{g_{\varepsilon}} C \eta^{j} \operatorname{Leb}\left(U_{n}\right) \leq C \overline{g_{\varepsilon}} \operatorname{Leb}\left(U_{n}\right) \frac{\eta}{\eta-1} \eta^{\alpha_{n}} .
\end{aligned}
$$

Now, observe that

$$
\begin{aligned}
& n \sum_{j=1}^{\left\lfloor n / k_{n}\right\rfloor} \mathbb{P}\left(U_{n} \cap f_{\underline{\omega}}^{-j}\left(U_{n}\right)\right) \leq n \sum_{j=\alpha_{n}}^{\left\lfloor n / k_{n}\right\rfloor} \mathbb{P}\left(\left\{(x, \underline{\omega}): x \in U_{n}, f_{\underline{\omega}}^{j}(x) \in U_{n}\right\}\right) \\
& \quad+n \sum_{j=1}^{\left\lfloor n / k_{n}\right\rfloor} \mathbb{P}\left(\left\{(x, \underline{\omega}): x \in U_{n}, R^{\underline{\omega}}\left(U_{n}\right) \leq \alpha_{n}\right\}\right):=I+I I .
\end{aligned}
$$

Let us start by estimating $I$, which will be dealt with as in Section 3. Taking $\psi=\phi=\mathbf{1}_{U_{n}}$ in (4.1) and since $\left\|\mathbf{1}_{U_{n}}\right\|_{\mathcal{C}} \leq C^{\prime}$, we get

$$
\begin{aligned}
\mathbb{P}\left(\left\{(x, \underline{\omega}): x \in U_{n}, f_{\underline{\omega}}^{j}(x) \in U_{n}\right\}\right) & \leq\left(\mu_{\varepsilon}\left(U_{n}\right)\right)^{2}+C\left\|\mathbf{1}_{U_{n}}\right\|_{\mathcal{C}}\left\|\mathbf{1}_{U_{n}}\right\|_{L^{1}\left(\mu_{\varepsilon}\right)} j^{-2} \\
& \leq\left(\mu_{\varepsilon}\left(U_{n}\right)\right)^{2}+C^{*} \mu_{\varepsilon}\left(U_{n}\right) j^{-2},
\end{aligned}
$$

where $C^{*}=C C^{\prime}>0$. Now observe that by definition of $U_{n}$ and (2.10), we have that $\mu_{\epsilon}\left(U_{n}\right) \sim \tau / n$. Using this observation together with the definition of $R_{n}^{\frac{\omega}{n}}$ and the estimate (4.5), it follows that there exists some constant $D>0$ such that

$$
\begin{gathered}
n \sum_{j=\alpha_{n}}^{\left\lfloor n / k_{n}\right\rfloor} \mathbb{P}\left(\left\{(x, \underline{\omega}): x \in U_{n}, f_{\underline{\omega}}^{j}(x) \in U_{n}\right\}\right) \leq n\left\lfloor\frac{n}{k_{n}}\right\rfloor \mu_{\varepsilon}\left(U_{n}\right)^{2}+n C^{*} \mu_{\varepsilon}\left(U_{n}\right) \sum_{j=\alpha_{n}}^{\left\lfloor n / k_{n}\right\rfloor} j^{-2} \\
\quad \leq \frac{\left(n \mu_{\varepsilon}\left(U_{n}\right)\right)^{2}}{k_{n}}+n C^{*} \mu_{\varepsilon}\left(U_{n}\right) \sum_{j=\alpha_{n}}^{\infty} j^{-2} \leq D\left(\frac{\tau^{2}}{k_{n}}+\tau \sum_{j=\alpha_{n}}^{\infty} j^{-2}\right) \underset{n \rightarrow \infty}{\longrightarrow} 0 .
\end{gathered}
$$

For the term $I I$, as $\mu_{\varepsilon}\left(U_{n}\right) \sim \tau / n$ and since $d \mu_{\varepsilon} / d$ Leb is bounded below and above by positive constants, there exists some positive constant $C^{*}>0$ so that

$$
\begin{aligned}
n \sum_{j=1}^{\left\lfloor n / k_{n}\right\rfloor} \mathbb{P}\left(\left\{(x, \underline{\omega}): x \in U_{n}, R^{\underline{\omega}}\left(U_{n}\right) \leq \alpha_{n}\right\}\right) & \leq \frac{n^{2}}{k_{n}} \mu_{\varepsilon}\left(U_{n}\right) C \overline{g_{\varepsilon}} \operatorname{Leb}\left(U_{n}\right) \frac{\eta}{\eta-1} \eta^{\alpha_{n}} \\
& \leq C^{*} \frac{\eta^{\alpha_{n}}}{k_{n}} \underset{n \rightarrow \infty}{\longrightarrow} 0 \text { by (4.3). }
\end{aligned}
$$

Proof of Corollary E. The only extra step we need to do is to check that $D_{3}\left(u_{n}\right)$ also holds. To do that we just have to slightly change the definition of $\psi$ that we 
used to prove $D_{2}\left(u_{n}\right)$ by using (4.1). Let $A \in \mathcal{R}$. We set

$$
\psi(x)=\int \mathbf{1}_{\bigcap_{i \in A \cap \mathbb{N}}\left\{f_{\underline{\underline{\omega}}}^{i}(x) \leq u_{n}\right\}} \mathrm{d} \theta_{\varepsilon}^{\mathbb{N}}(\underline{\tilde{\omega}}) .
$$

The rest of the proof follows exactly as in the proof of $D_{2}\left(u_{n}\right)$ in the proof of Theorem D,

\subsection{Laws of rare events for specific randomly perturbed systems.}

4.2.1. Expanding and piecewise expanding maps on the circle with a finite number of discontinuities. We give a general definition from [30] of piecewise expanding maps on the circle which also includes the particular case of the continuous expanding maps:

(1) there exist $\ell \in \mathbb{N}_{0}$ and $0=a_{0}<a_{1}<\cdots<a_{\ell}=1=0=a_{0}$ for which the restriction of $f$ to each $\Xi_{i}=\left(a_{i-1}, a_{i}\right)$ is of class $C^{1}$, with $|D f(x)|>0$ for all $x \in \Xi_{i}$ and $i=1, \ldots, \ell$. In addition, for all $i=1, \ldots, \ell, g_{\Xi_{i}}=1 /|D f|_{\Xi_{i}} \mid$ has bounded variation for $i=1, \ldots, \ell$.

We assume that $\left(\left.f\right|_{\Xi_{i}}\right)$ and $g_{\Xi_{i}}$ admit continuous extensions to $\Xi_{i}=\left[a_{i-1}, a_{i}\right]$, for each $i=1, \ldots, \ell$. Since modifying the values of a map over a finite set of points does not change its statistical properties, we may assume that $f$ is either left-continuous or right-continuous (or both) at $a_{i}$, for each $i=1, \ldots, \ell$ (possibly for all $i$ 's at the same time). Then let $\mathcal{P}^{(1)}$ be some partition of $\mathcal{S}^{1}$ into intervals $\Xi$ such that $\Xi \subset \Xi_{i}$ for some $i$ and $(f \mid \Xi)$ is continuous. Furthermore, for $n \geq 1$, let $\mathcal{P}^{(n)}$ be the partition of $\mathcal{S}^{1}$ such that $\mathcal{P}^{(n)}(x)=\mathcal{P}^{(n)}(y)$ if and only if $\mathcal{P}^{(1)}\left(f^{j}(x)\right)=\mathcal{P}^{(1)}\left(f^{j}(y)\right)$ for all $0 \leq j<n$. Given $\Xi \in \mathcal{P}^{(n)}$, denote $g_{\Xi}^{(n)}=1 /\left|D f^{n}\right|_{\Xi} \mid$. Then

(2) there exist constants $C_{1}>0, \lambda_{1}<1$ such that $\sup g_{\Xi}^{(n)} \leq C_{1} \lambda_{1}^{n}$ for all $\Xi \in \mathcal{P}^{(n)}$ and all $n \geq 1$

(3) for every subinterval $J$ of $\mathcal{S}^{1}$, there exists some $n \geq 1$ such that $f^{n}(J)=\mathcal{S}^{1}$.

According to [30, Proposition 3.15], one has exponential decay of correlations for randomly perturbed systems derived from maps satisfying conditions $(1)-(3)$ above, taking $\mathcal{C}$ as the space of functions with bounded variation $(B V)$; i.e., given $\varphi$ in $B V$ and $\psi \in L^{1}(\mathrm{Leb})$,

$$
\left|\int\left(\mathcal{U}_{\varepsilon} \psi\right) \varphi \mathrm{dLeb}-\int \psi \mathrm{d} \mu_{\varepsilon} \int \varphi \mathrm{dLeb}\right| \leq C \lambda^{n}\|\varphi\|_{B V}\|\psi\|_{L^{1}(\mathrm{Leb})},
$$

where $0<\lambda<1$ and $C>0$ is a constant independent of both $\varphi, \psi$.

Hence, in the particular case of $f$ being a continuous expanding map of the circle, (4.7), Theorem D, and Corollaries E and F allow us to obtain

Corollary 4.1. Let $f: \mathcal{S}^{1} \rightarrow \mathcal{S}^{1}$ be a continuous expanding map satisfying (1) - (3) above, which is randomly perturbed as in (2.2) with noise distribution given by (2.1). For any point $\zeta \in \mathcal{M}$, consider that $X_{0}, X_{1}, \ldots$ is defined as in (2.7) and let $u_{n}$ be such that (2.10) holds. Then the stochastic process $X_{0}, X_{1}, \ldots$ satisfies $D_{2}\left(u_{n}\right), D_{3}\left(u_{n}\right)$ and $D^{\prime}\left(u_{n}\right)$, which implies that we have an $E V L$ for $M_{n}$ such that $\bar{H}(\tau)=\mathrm{e}^{-\tau}$ and we have exponential HTS/RTS for balls around $\zeta$. Moreover, the REPP $N_{n}$ defined in (2.20) is such that $N_{n} \stackrel{d}{\rightarrow} N$, as $n \rightarrow \infty$, where $N$ denotes a Poisson Process with intensity 1. 
In the proof of Theorem $\mathrm{D}$, we used the continuity of the map, in particular, in (4.4). However, we can adapt the argument in order to allow a finite number of discontinuities for expanding maps of the circle.

Proposition 4.2. Let $f: \mathcal{S}^{1} \rightarrow \mathcal{S}^{1}$ be a map satisfying conditions (1) - (3) above, which is randomly perturbed as in (2.2) with noise distribution given by (2.1). For any point $\zeta \in \mathcal{M}$, consider that $X_{0}, X_{1}, \ldots$ is defined as in (2.7) and let $u_{n}$ be such that (2.10) holds. Then the stochastic process $X_{0}, X_{1}, \ldots$ satisfies $D_{2}\left(u_{n}\right), D_{3}\left(u_{n}\right)$ and $D^{\prime}\left(u_{n}\right)$, which implies that we have an $E V L$ for $M_{n}$ such that $\bar{H}(\tau)=\mathrm{e}^{-\tau}$ and we have exponential HTS/RTS for balls around $\zeta$. Moreover, the REPP $N_{n}$ defined in (2.20) is such that $N_{n} \stackrel{d}{\rightarrow} N$, as $n \rightarrow \infty$, where $N$ denotes a Poisson Process with intensity 1.

Proof. The proof of $D_{2}\left(u_{n}\right)$ follows from (4.7) as in the continuous case. Regarding the proof of $D^{\prime}\left(u_{n}\right)$, in order to use the same arguments as in the continuous case, we want to avoid coming close to the discontinuity points along the random orbit of $\zeta$ (up to time $\alpha_{n}$ ). Since there are finitely many discontinuity points, say $\xi_{i}$ 's for $i=1, \ldots, \ell$, we can control this by asking for some "safety regions" around each of them. By doing so, we ensure that the random orbit of $\zeta$ is sufficiently far away from the $\xi_{i}$ 's so that the iterates of $U_{n}$ consist of only one connected component. We can formulate these "safety regions" as

$$
\operatorname{dist}\left(f_{\underline{\omega}}^{j}(\zeta), \xi_{i}\right)>2 \eta^{j}\left|U_{n}\right| \text { for all } i=1, \ldots, \ell .
$$

Now, we make an estimate on the $\underline{\omega}$ 's that take the orbit of $\zeta$ too close to the discontinuity points as well as close to $\zeta$ itself, and our aim is to show that the $\theta_{\varepsilon}^{\mathbb{N}}$ measure of this set is small. Let us set $\xi_{0}=\zeta$ to simplify the notation. Then, $\left\{\underline{\omega}: R^{\underline{\omega}}\left(U_{n}\right) \leq \alpha_{n}\right\} \subset \bigcup_{j=1}^{\alpha_{n}} \bigcup_{i=0}^{\ell}\left\{\underline{\omega}: f_{\underline{\omega}}^{j}(\zeta) \in B_{2 \eta^{j}\left|U_{n}\right|}\left(\xi_{i}\right)\right\}$. Thus, we have

$$
\begin{aligned}
& \theta_{\varepsilon}^{\mathbb{N}}\left(\left\{\underline{\omega}: R^{\underline{\omega}}\left(U_{n}\right) \leq \alpha_{n}\right\}\right) \\
& \quad \leq \sum_{i=0}^{\ell} \sum_{j=1}^{\alpha_{n}} \int \theta_{\varepsilon}\left(\left\{\omega_{j}: f\left(f_{\underline{\omega}}^{j-1}(\zeta)\right)+\omega_{j} \in B_{2 \eta^{j}\left|U_{n}\right|}\left(\xi_{i}\right)\right\}\right) \mathrm{d} \theta_{\varepsilon}^{\mathbb{N}} \\
& \quad \leq \sum_{i=0}^{\ell} \sum_{j=1}^{\alpha_{n}} \overline{g_{\varepsilon}}\left|B_{2 \eta^{j}\left|U_{n}\right|}\left(\xi_{i}\right)\right|=\sum_{i=0}^{\ell} \sum_{j=1}^{\alpha_{n}} \overline{g_{\varepsilon}} 4 \eta^{j}\left|U_{n}\right| \leq 4(\ell+1) \overline{g_{\varepsilon}}\left|U_{n}\right| \frac{\eta}{\eta-1} \eta^{\alpha_{n}} .
\end{aligned}
$$

The proof now follows the same lines as the proof of Theorem $\mathrm{D}$ and Corollary $\mathrm{E}$

4.2.2. Expanding and piecewise expanding maps in higher dimensions. Let us now consider the multidimensional piecewise expanding systems defined in Section 3.2 .2 but only with a finite number, $K$, of domains of local injectivity; moreover, let us restrict ourselves to a mixing component which, for simplicity, we will take as the whole space $Z$; we will take $\mu$ as the unique absolutely continuous invariant measure with density $h$. In addition, we ask each $\partial Z_{i}$ to be included in piecewise $C^{1}$ codimension- 1 embedded compact submanifolds and for $Z(f)=$ $\sup _{x} \sum_{i=1}^{K} \#\left\{\right.$ smooth pieces intersecting $\partial Z_{i}$ containing $\left.x\right\}$

$$
s^{\alpha}+\frac{4 s}{1-s} Z(f) \frac{\gamma_{N-1}}{\gamma_{N}}<1
$$


where $\gamma_{N}$ is the $N$-volume of the $N$-dimensional unit ball of $\mathbb{R}^{N}$. Then, we know that by Lemma 2.1 in 28 , item (4) in Definition 3.5 is satisfied 3 Notice that formula (4.9) gives an exponential decay of correlations for the adapted pair: $L^{1}$ functions against functions in the quasi-Hölder space $V_{\alpha}$.

We perturb again this kind of map with additive noise by asking that the image of $Z$ is strictly included in $Z$. We will also require that the density $h$ is bounded from below by the positive constant $h_{m}$. We will now prove the exponential decay of correlations for the random evolution operator $\mathcal{U}_{\varepsilon}$, by using the perturbation theory in [21, which we will also quote and use later on in Section 5.2. This theory ensures us that the perturbed Perron-Frobenius operator $\mathcal{P}_{\epsilon}$ is mixing on the adapted pair $\left(L^{1}, V_{\alpha}\right)$ whenever we have:

(i) a uniform Lasota-Yorke inequality for $\mathcal{P}_{\epsilon}$, i.e., all the constants in that inequality are independent of the noise $\varepsilon$,

(ii) the closeness property (see also hypothesis $\mathbf{H 4}$ in Section 5.2 below): there exists a monotone upper semi-continuous function $p: \Omega \rightarrow[0, \infty)$ such that $\lim _{\varepsilon \rightarrow 0} p_{\varepsilon}=0$ and $\forall \varphi \in V_{\alpha}, \forall \varepsilon \in \Omega:\left\|\mathcal{P} \varphi-\mathcal{P}_{\varepsilon} \varphi\right\|_{1} \leq p_{\varepsilon}\|\varphi\|_{\alpha}$.

Condition (i) follows easily by observing that the derivatives of the original and of the perturbed maps are the same, which does not change the contraction factor $s$, and the multiplicity of the boundaries' intersection, $Z(f)$, is invariant too. Finally we invoke the observation written in the preceding footnote. Therefore the Perron-Frobenius operators $\mathcal{P}_{\omega}$ associated to the perturbed maps $f_{\omega}$ verify the same Lasota-Yorke inequality and therefore the same is true for $\mathcal{P}_{\varepsilon}$.

Our next step is to prove condition (ii); in particular, we have

Proposition 4.3. There exists a constant $C$ such that for any $\varphi \in V_{\alpha}$ we have

$$
\left\|\mathcal{P} \varphi-\mathcal{P}_{\varepsilon} \varphi\right\|_{1} \leq C \varepsilon^{\alpha}\|\varphi\|_{\alpha} .
$$

Proof. We have

$$
\left\|\mathcal{P} \varphi-\mathcal{P}_{\varepsilon} \varphi\right\|_{1} \leq \int_{Z} \int_{\Omega}\left|\mathcal{P}_{\omega} \varphi(x)-\mathcal{P} \varphi(x)\right| \mathrm{d} \theta_{\varepsilon}(\omega) \mathrm{d} x .
$$

Putting $G(x)=\frac{1}{|\operatorname{det} D f(x)|}$, we can write

$$
\begin{aligned}
& \left|\mathcal{P}_{\omega} \varphi(x)-\mathcal{P} \varphi(x)\right| \\
& \leq \sum_{Z_{i}, i=1, \ldots, K}\left|\varphi\left(f_{i}^{-1} x\right) G\left(f_{i}^{-1} x\right) \mathbf{1}_{f Z_{i}}(x)-\varphi\left(f_{\omega, i}^{-1} x\right) G\left(f_{i}^{-1} x\right) \mathbf{1}_{f_{\omega} Z_{i}}(x)\right| \\
& \quad+\sum_{Z_{i}, i=1, \ldots, K}\left|\varphi\left(f_{\omega, i}^{-1} x\right)\right|\left|G\left(f_{i}^{-1} x\right)-G\left(f_{\omega, i}^{-1} x\right)\right| \mathbf{1}_{f_{\omega} Z_{i}}(x) \\
& \quad:=I+I I,
\end{aligned}
$$

where $f_{\omega}(x)=f(x)+\omega$ and $\omega$ is a vector in $\mathbb{R}^{N}$ with each component being less than $\varepsilon$ in modulus. Moreover, $f_{\omega, i}^{-1}$ denotes the inverse of the restriction of $f_{\omega}$ to $Z_{i}$, which is denoted by $f_{\omega, i}$ itself. We now bound the first sum, $I$, in (4.10) by considering the following two cases.

\footnotetext{
${ }^{3}$ The inequality (4.9) ensures that for the unperturbed map the quantity $\eta\left(\varepsilon_{1}\right)<1$; see the definition of this quantity after the formula (3.3). The value of $\eta\left(\varepsilon_{1}\right)$ is one of the constants in the Lasota-Yorke inequality (see item (i) below), and we will require that it is independent of the noise. This will be the case for the additive noise since the determinant of the perturbed maps will not change, and this is what is used in (3.3) to control the Lebesgue measure of $f_{i}^{-1} B_{\varepsilon}\left(\partial f Z_{i}\right)$. The other factor in the Lasota-Yorke inequality is also given in terms of the quantity (3.3).
} 
(i) Let us suppose first that $x \in f Z_{i} \cap f_{\omega, i} Z_{i}$. Then since both $f$ and $f_{\omega, i}$ are injective, there will be two points, $y_{i}$ and $y_{\omega, i}$, in $Z_{i}$ such that $x=f\left(y_{i}\right)=$ $f_{\omega, i}\left(y_{\omega, i}\right)=f\left(y_{\omega, i}\right)+\omega$. This immediately implies that $\operatorname{dist}\left(y_{i}, y_{\omega, i}\right) \leq s \sqrt{N} \varepsilon$, if dist is the Euclidean distance. For such an $x$ we continue to bound the first summand, $I$, in (4.10) as:

$$
I \leq \sum_{Z_{i}, i=1, \ldots, K} G\left(f_{i}^{-1} x\right) \operatorname{osc}\left(\varphi, B_{s \sqrt{N} \varepsilon}\left(f_{i}^{-1}(x)\right)\right) \mathbf{1}_{f Z_{i}}(x) .
$$

By integrating over $Z$ we get

$$
\begin{aligned}
\int_{Z}( & \left.\sum_{Z_{i}, i=1, \ldots, K} G\left(f_{i}^{-1} x\right) \operatorname{osc}\left(\varphi, B_{s \sqrt{N} \varepsilon}\left(f_{i}^{-1}(x)\right)\right) \mathbf{1}_{f Z_{i}}(x)\right) \mathrm{d} x \\
= & \int_{Z} \mathcal{P}\left(\operatorname{osc}\left(\varphi, B_{s \sqrt{N} \varepsilon}(x)\right)\right) \mathrm{d} x \\
= & \int_{Z} \operatorname{osc}\left(\varphi, B_{s \sqrt{N} \varepsilon}(x)\right) \mathrm{d} x \leq(s \sqrt{N} \varepsilon)^{\alpha}|\varphi|_{\alpha} .
\end{aligned}
$$

(ii) We now consider the case when $x \in f Z_{i} \Delta f_{\omega, i} Z_{i}$; the Lebesgue measure of this last set is bounded by $\varepsilon$ times the codimension- 1 volume of $\partial f Z_{i}$ : let $r$ denote the maximum of those volumes for $i=1, \cdots, k$. Thus we get

$$
\int_{Z}\left|\mathcal{P}_{\omega} \varphi(x)-\mathcal{P} \varphi(x)\right| \mathrm{d} x \leq r \varepsilon\|\varphi\|_{\infty}\|\mathcal{P} 1\|_{\infty} .
$$

We notice that the inclusion $V_{\alpha} \hookrightarrow L_{m}^{\infty}$ is bounded; namely, there exists $c_{v}$ such that $\|\varphi\|_{\infty} \leq c_{v}\|\varphi\|_{\alpha}$. We therefore continue (4.11) as

$$
\text { (4.11) } \leq r \varepsilon c_{v}\|\varphi\|_{\alpha}\left\|\mathcal{P} \frac{h}{h}\right\|_{\infty} \leq r \varepsilon c_{v}\|\varphi\|_{\alpha} \frac{\|h\|_{\infty}}{h_{m}} \text {. }
$$

We now come to the second summand, $I I$, in (4.10). We begin by observing that

$$
\begin{aligned}
\left|G\left(f_{i}^{-1} x\right)-G\left(f_{\omega, i}^{-1} x\right)\right| & =\left|\frac{1}{\left|\operatorname{det} D f\left(f_{i}^{-1} x\right)\right|}-\frac{1}{\left|\operatorname{det} D f\left(f_{\omega, i}^{-1} x\right)\right|}\right| \\
& =|| \operatorname{det} D f_{i}^{-1}(x)|-| \operatorname{det} D f_{i}^{-1}(z)|| \\
& \leq\left|\operatorname{det} D f_{i}^{-1}(x)-\operatorname{det} D f_{i}^{-1}(z)\right|,
\end{aligned}
$$

where $z=f\left(y_{\omega, i}\right)$ and $\operatorname{dist}(x, z) \leq \sqrt{N} \varepsilon$. By using the Hölder assumption (2) in Definition 3.5, we have

$$
\begin{aligned}
I I & \leq c(\sqrt{N} \varepsilon)^{\alpha} \sum_{Z_{i}, i=1, \ldots, K}\left|\varphi\left(f_{\omega, i}^{-1} x\right)\right|\left|\operatorname{det} D f_{i}^{-1}(z)\right| \mathbf{1}_{f_{\omega} Z_{i}}(x) \\
& \leq c(\sqrt{N} \varepsilon)^{\alpha} \sum_{Z_{i}, i=1, \ldots, K}\left|\varphi\left(f_{\omega, i}^{-1} x\right)\right| \frac{1}{\left|\operatorname{det} D f\left(f_{\omega, i}^{-1}(x)\right)\right|} \mathbf{1}_{f_{\omega} Z_{i}}(x) .
\end{aligned}
$$

By integrating over $Z$ we get the contribution

$$
c(\sqrt{N} \varepsilon)^{\alpha} \int_{Z} \mathcal{P}_{\omega}(|\varphi|) \mathrm{d} x \leq c(\sqrt{N} \varepsilon)^{\alpha} \int_{Z}|\varphi| \mathrm{d} x \leq c(\sqrt{N} \varepsilon)^{\alpha}\|\varphi\|_{L^{1}(\text { Leb })} .
$$

In conclusion we get $\left\|\mathcal{P} \varphi-\mathcal{P}_{\varepsilon} \varphi\right\|_{1} \leq C \varepsilon^{\alpha}\|\varphi\|_{\alpha}$, where the constant $C$ collects the various constants introduced above. 
As a consequence of Proposition 4.3 we obtain exponential decay of correlations of quasi-Hölder functions (in $V_{\alpha}$ ), against $L^{1}$ functions, in particular, for uniformly expanding maps on the torus $\mathbb{T}^{d}$. Since $\mathbf{1}_{U_{n}} \in V_{\alpha},\left\|\mathbf{1}_{U_{n}}\right\|_{\alpha}$ is uniformly bounded by above, then it follows by Theorem $\mathrm{D}$ and Corollary $\mathrm{E}$ that

Corollary 4.4. Let $f: \mathbb{T}^{d} \rightarrow \mathbb{T}^{d}$ be a $C^{2}$ uniformly expanding map on $\mathbb{T}^{d}$, which is randomly perturbed as in (2.2) with noise distribution given by (2.1). For any point $\zeta \in \mathcal{M}$, consider that $X_{0}, X_{1}, \ldots$ is defined as in (2.7) and let $u_{n}$ be such that (2.10) holds. Then the stochastic process $X_{0}, X_{1}, \ldots$ satisfies $D_{2}\left(u_{n}\right), D_{3}\left(u_{n}\right)$ and $D^{\prime}\left(u_{n}\right)$, which implies that we have an $E V L$ for $M_{n}$ such that $\bar{H}(\tau)=\mathrm{e}^{-\tau}$ and we have exponential HTS/RTS for balls around $\zeta$. Moreover, the REPP $N_{n}$ defined in (2.20) is such that $N_{n} \stackrel{d}{\rightarrow} N$, as $n \rightarrow \infty$, where $N$ denotes a Poisson Process with intensity 1.

As in the previous case of maps on the circle, we may adapt the argument used in the continuous case to consider more general piecewise expanding maps of Definition 3.5, as long as there is a finite number of domains of local injectivity.

Proposition 4.5. Suppose that $(Z, f, \mu)$ is a topologically mixing multidimensional piecewise expanding system as in Definition 3.5, and $\mu$ is the a.c.i.p. with a RadonNikodym density bounded away from 0 . We assume that there are $K \in \mathbb{N}$ domains of injectivity of the map and there exists $\eta>1$ such that for all $i=1, \ldots, K$ and all $x, y \in Z_{i}$ we have $\operatorname{dist}(f(x), f(y)) \leq \eta \operatorname{dist}(x, y)$. Consider that such a map is randomly perturbed with additive noise as in (2.2) with noise distribution given by (2.1) and such that the image of $Z$ is strictly included in $Z$. For any point $\zeta \in \mathcal{M}$, consider that $X_{0}, X_{1}, \ldots$ is defined as in (2.7) and let $u_{n}$ be such that (2.10) holds. Then the stochastic process $X_{0}, X_{1}, \ldots$ satisfies $D_{2}\left(u_{n}\right), D_{3}\left(u_{n}\right)$ and $D^{\prime}\left(u_{n}\right)$, which implies that we have an EVL for $M_{n}$ such that $\bar{H}(\tau)=\mathrm{e}^{-\tau}$ and we have exponential HTS/RTS for balls around $\zeta$. Moreover, the REPP $N_{n}$ defined in (2.20) is such that $N_{n} \stackrel{d}{\rightarrow} N$, as $n \rightarrow \infty$, where $N$ denotes a Poisson Process with intensity 1.

Proof. Previously, for maps on the circle, by putting some "safety regions" around the discontinuity points we guaranteed that the iterates of $f_{\omega}^{j}\left(U_{n}\right), j=0,1, \ldots, \alpha_{n}$, had one connected component. Since in this case the border of the domains of injectivity are codimension-1 submanifolds instead of single points (as in the onedimensional case), we must proceed to a more thorough analysis. To that end, for each $\underline{\omega}$, for $j=1$ let $1 \leq l_{1} \leq K$ be the number of intersections with non-empty interior between $f_{\underline{\omega}}\left(U_{n}\right)$ and $Z_{i}$, with $i=1, \ldots, K$. For each $\ell=1, \ldots, l_{1}$, let $i_{\ell}$ denote the index of the partition element $Z_{i_{\ell}}$ for which such intersection has nonempty interior, define $U_{n}^{(1, \ell)}:=f_{\underline{\omega}}\left(U_{n}\right) \cap Z_{i_{\ell}}$ and let $\zeta_{1, \ell}$ be a point in the interior of $U_{n}^{(1, \ell)}$. For any $j=2, \ldots, \alpha_{n}$, given the sets $U_{n}^{(j-1, k)}$, with $k=1, \ldots, l_{j-1}$, let $l_{j}$ be the total number of intersections of non-empty interior between $f_{\sigma^{j-1}(\underline{\omega})}\left(U_{n}^{(j-1, k)}\right)$ and $Z_{i}$, with $i=1, \ldots, K$. For each $\ell=1, \ldots, l_{j}$, let $i_{\ell}$ denote the index of the partition element $Z_{i_{\ell}}$ and $k_{\ell}$ the super index of the sets $U_{n}^{(j-1, k)}$ for which the intersection between $f_{\sigma^{j-1}(\underline{\omega})}\left(U_{n}^{\left(j-1, k_{\ell}\right)}\right)$ and $Z_{i_{\ell}}$ has non-empty interior, define $U_{n}^{(j, \ell)}=f_{\sigma^{j-1}(\underline{\omega})}\left(U_{n}^{\left(j-1, k_{\ell}\right)}\right) \cap Z_{i_{\ell}}$ and let $\zeta_{j, \ell}$ be a point in the interior of $U_{n}^{(j, \ell)}$. 
In order to avoid the first return time to $U_{n}$ occurring before $\alpha_{n}$ in a similar way to the previous proofs, we require that

$$
\operatorname{dist}\left(f_{\sigma^{j-1}(\underline{\omega})}\left(\zeta_{j-1, \ell}\right), \zeta\right)>2 \eta^{j}\left|U_{n}\right| \text { for all } j=2, \ldots, \alpha_{n}, \ell=1, \ldots, l_{j-1} .
$$

Note that, as in the proof of Theorem $\mathrm{D}$, for any $\underline{\omega} \in \Omega$, we have $\left|U_{n}^{(j, \ell)}\right| \leq \eta^{j}\left|U_{n}\right|$. This implies that

(4.13) if $\operatorname{dist}\left(f_{\sigma^{j-1}(\underline{\omega})}\left(\zeta_{j-1, \ell}\right), \zeta\right)>2 \eta^{j}\left|U_{n}\right|>\left|U_{n}\right|+\eta^{j}\left|U_{n}\right|$, then $U_{n}^{(j, \ell)} \cap U_{n}=\emptyset$.

Note that, by equation (4.13), if (4.12) holds, then clearly $R^{\omega}\left(U_{n}\right)>\alpha_{n}$. Hence, letting $l_{0}=1$ and $\zeta_{0,1}=\zeta$, we may write that

$$
\left\{\underline{\omega}: R^{\underline{\omega}}\left(U_{n}\right) \leq \alpha_{n}\right\} \subset \bigcup_{j=1}^{\alpha_{n}} \bigcup_{\ell=1}^{l_{j-1}}\left\{\underline{\omega}: f_{\sigma^{j-1}(\underline{\omega})}\left(\zeta_{j-1, \ell}\right) \in B_{2 \eta^{j}\left|U_{n}\right|}(\zeta)\right\} .
$$

Recalling that $l_{j} \leq K^{j}$, for all $j=1, \ldots, \alpha_{n}$, it follows that there exists some $C>0$ such that

$$
\begin{aligned}
\theta_{\varepsilon}^{\mathbb{N}}\left(\left\{\underline{\omega}: R^{\underline{\omega}}\left(U_{n}\right) \leq \alpha_{n}\right\}\right) & \leq \sum_{j=1}^{\alpha_{n}} \sum_{\ell=1}^{l_{j-1}} \int \theta_{\varepsilon}\left(\left\{\omega_{j}: f\left(\zeta_{j-1, \ell}\right)+\omega_{j} \in B_{2 \eta^{j}\left|U_{n}\right|}(\zeta)\right\}\right) \mathrm{d} \theta_{\varepsilon}^{\mathbb{N}} \\
& \leq \sum_{j=1}^{\alpha_{n}} \sum_{\ell=1}^{l_{j-1}} \overline{g_{\varepsilon}} \operatorname{Leb}\left(B_{2 \eta^{j}\left|U_{n}\right|}(\zeta)\right) \\
& \leq \sum_{j=1}^{\alpha_{n}} K^{j} \overline{g_{\varepsilon}} C \eta^{j} \operatorname{Leb}\left(U_{n}\right) \leq C \overline{g_{\varepsilon}} \operatorname{Leb}\left(U_{n}\right) \frac{\eta K}{\eta K-1}(\eta K)^{\alpha_{n}} .
\end{aligned}
$$

Now, the proof follows in exactly the same way as the proof of Theorem $\mathrm{D}$ and Corollary E. except that in the final estimate (4.6), $\eta$ should be replaced by $\eta K$, which will not make any difference by the choice of $\alpha_{n}$ defined in (4.3).

\section{EXTREMES FOR RANDOM DYNAMICS FROM A SPECTRAL APPROACH}

In this section, we want to prove our results for the random case using another approach introduced by Keller in [20. His technique is based on an eigenvalue perturbation formula which was given in 21 under a certain number of assumptions that we recall in the first subsection and adapt to our situation. We check those assumptions in Section 5.3 for a large class of maps of the interval whose properties are listed in the conditions (H1-H5). Possible generalisations deserve to be investigated, and we point out here a major difficulty in higher dimensions. In this case one should control (any kind of) variation/oscillation on the boundaries of the preimages of the complement of balls (the set $U_{m}^{c}$ in the proof of Proposition 5.2 below; it is important that such variation/oscillation grows at most sub-exponentially). To sum up, the direct technique introduced in Section 4 and the spectral one in this section are complementary. The direct technique is easily adapted to higher dimensions but it requires assumptions on the noise in order to control the short returns (see the quantity $R \underline{\omega}\left(U_{n}\right)$ in Proposition 4.5 ), which follows easily for additive noise. The spectral technique is an alternative method and for the moment particularly adapted to the 1-D case, and, as we will see in a moment, the noise could be chosen in a quite general way to prove the existence of the EI, formula (5.7). Instead, if we want to characterise such an EI and show that it is always 
equal to 1, we need to consider special classes of uniformly expanding maps, and particularly the noise should be chosen as additive and with a continuous distribution (Proposition 5.3). The fact that the existence of EI follows for general classes of noises is clear by looking at the proof of Proposition 5.2. Indeed, what is really necessary is that the derivatives of the randomly chosen maps are close enough to each other in order to guarantee the uniformity of the Lasota-Yorke inequality for the perturbed Perron-Frobenius operator. This could be achieved quite widely and with discrete distributions as well. Nevertheless, in order to make the exposition simpler and coherent with the previous sections, we will consider additive noise, together with any kind of distribution to prove Proposition 5.2 and with absolutely continuous distributions to prove Proposition 5.3 .

5.1. The setting. Given a Banach space $(V,\|\cdot\|)$, and a set of parameters $E$ which is equipped with some topology, let us suppose there are $\lambda_{\varepsilon} \in \mathbb{C}, \varphi_{\varepsilon} \in V, \nu_{\varepsilon} \in V^{\prime}$ ( $V^{\prime}$ denotes the dual of $\mathrm{V}$ ) and linear operators $P_{\varepsilon}, Q_{\varepsilon}: V \rightarrow V$ such that

$$
\begin{gathered}
\left.\lambda_{\varepsilon}^{-1} P_{\varepsilon}=\varphi_{\varepsilon} \otimes \nu_{\varepsilon}+Q_{\varepsilon} \text { (assume } \lambda_{0}=1\right), \\
P_{\varepsilon}\left(\varphi_{\varepsilon}\right)=\lambda_{\varepsilon} \varphi_{\varepsilon}, \nu_{\varepsilon} P_{\varepsilon}=\lambda_{\varepsilon} \nu_{\varepsilon}, Q_{\varepsilon}\left(\varphi_{\varepsilon}\right)=0, \nu_{\varepsilon} Q_{\varepsilon}=0, \\
\sum_{n=0}^{\infty} \sup _{\varepsilon \in E}\left\|Q_{\varepsilon}^{n}\right\|=: C_{1}<\infty, \\
\exists C_{2}>0, \forall \varepsilon \in E: \nu_{0}\left(\varphi_{\varepsilon}\right)=1 \text { and }\left\|\varphi_{\varepsilon}\right\| \leq C_{2}<\infty, \\
\lim _{\varepsilon \rightarrow 0}\left\|\nu_{0}\left(P_{0}-P_{\varepsilon}\right)\right\|=0, \\
\left\|\nu_{0}\left(P_{0}-P_{\varepsilon}\right)\right\| \cdot\left\|\left(P_{0}-P_{\varepsilon}\right) \varphi_{0}\right\| \leq \text { const } \cdot\left|\Delta_{\varepsilon}\right|,
\end{gathered}
$$

where

$$
\Delta_{\varepsilon}:=\nu_{0}\left(\left(P_{0}-P_{\varepsilon}\right)\left(\varphi_{0}\right)\right)
$$

Under these assumptions, Keller and Liverani obtained the following formula as the main result in [21]:

$$
1-\lambda_{\varepsilon}=\Delta_{\varepsilon} \vartheta(1+o(1)) \text { in the limit as } \varepsilon \rightarrow 0,
$$

where $\vartheta$ is said to be a constant to take care of short time correlations, which is later identified as the extremal index in extreme value theory context as mentioned in [20, Section 1.2]. Actually $\vartheta$ is given by an explicit and, in some cases, computable formula, and, in fact, we will be able to compute it for our random systems. This formula is the content of Theorem 2.1 in [21] and states that under the above assumptions, in particular when $\Delta_{\varepsilon} \neq 0$, for $\varepsilon$ small enough, and whenever the following limit exists,

$$
q_{k}:=\lim _{\varepsilon \rightarrow 0} q_{k, \varepsilon}:=\lim _{\varepsilon \rightarrow \infty} \frac{\nu_{0}\left(\left(P_{0}-P_{\varepsilon}\right) P_{\varepsilon}^{k}\left(P_{0}-P_{\varepsilon}\right)\left(\varphi_{0}\right)\right)}{\Delta_{\varepsilon}}
$$

we have

$$
\lim _{\varepsilon \rightarrow 0} \frac{1-\lambda_{\varepsilon}}{\Delta_{\varepsilon}}=\vartheta:=1-\sum_{k=0}^{\infty} q_{k} .
$$


We now state equivalent ways to verify assumptions (5.1)-(5.6); we refer to 20] for the details.

(A1) There are constants $A>0, B>0, D>0$ and a second norm $|\cdot|_{\omega} \leq\|\cdot\|$ on $V$ (it is enough to be a seminorm) such that:

$$
\begin{gathered}
\forall \varepsilon \in E, \forall \psi \in V, \forall n \in \mathbb{N}:\left|P_{\varepsilon}^{n} \psi\right|_{\omega} \leq D|\psi|_{\omega}, \\
\exists \alpha \in(0,1), \forall \varepsilon \in E, \forall \psi \in V, \forall n \in \mathbb{N}:\left\|P_{\varepsilon}^{n} \psi\right\| \leq A \alpha^{n}\|\psi\|+B|\psi|_{\omega} .
\end{gathered}
$$

Moreover the closed unit ball of $(V,\|\cdot\|)$ is $|\cdot|_{\omega}$-compact.

(A2) The unperturbed operator verifies the mixing condition

$$
P=\varphi \otimes \nu+Q_{0}\left(\text { assume } \lambda_{0}=1\right) .
$$

(A3) $\exists C>0$ such that

$$
\eta_{\varepsilon}:=\sup _{\|\psi\| \leq 1}\left|\int\left(P_{0}-P_{\varepsilon}\right) \psi \mathrm{d} \nu_{0}\right| \rightarrow 0, \text { as } \varepsilon \rightarrow 0
$$

(A4) and

$$
\eta_{\varepsilon}\left\|\left(P_{0}-P_{\varepsilon}\right) \varphi_{0}\right\| \leq C \Delta_{\varepsilon} .
$$

Keller called this framework Rare events Perron-Frobenius operators, REPFO. We will construct a perturbed Perron-Frobenius operator which satisfies the previous assumptions and which will give us information on extreme value distributions and statistics of first returns to small sets.

Before continuing, we should come back to our extreme distributions, namely to the quantity $\left\{M_{m} \leq u_{m}\right\}=\left\{r_{\left\{\phi>u_{m}\right\}}>m\right\}$ where $U_{m}:=\left\{\phi>u_{m}\right\}$ is a topological ball shrinking to the point $\zeta$ (see (2.12); we changed $U_{n}$ into $U_{m}$ here). Now we consider the first time $r \frac{\omega}{U_{m}}(x)$ where the point $x$ enters $U_{m}$ under the realization $\underline{\omega}$, namely under the composition $\cdots \circ f_{\omega_{k}} \circ f_{\omega_{k-1}} \circ \cdots \circ f_{\omega_{1}}(x)$. For simplicity we indicate it by $r \frac{\omega}{m}(x)$ and consider its annealed distribution:

$$
\left(\mu_{\varepsilon} \times \theta_{\varepsilon}^{\mathbb{N}}\right)\left((x, \underline{\omega}): r \frac{\omega}{m}(x)>m\right)=\left(\mu_{\varepsilon} \times \theta_{\varepsilon}^{\mathbb{N}}\right)\left(M_{m} \leq u_{m}\right) .
$$

Let us write the measure on the left hand side of (5.14) in terms of integrals: it is given by

$$
\iint_{\left\{r_{m}^{\omega}>m\right\}} \mathrm{d}\left(\mu_{\varepsilon} \times \theta_{\varepsilon}^{\mathbb{N}}\right)=\iint h_{\varepsilon} \mathbf{1}_{U_{m}^{c}}(x) \mathbf{1}_{U_{m}^{c}}\left(f_{\omega_{1}} x\right) \cdots \mathbf{1}_{U_{m}^{c}}\left(f_{\omega_{m-1}} \circ \cdots \circ f_{\omega_{1}} x\right) \mathrm{dLeb} \mathrm{d} \theta_{\varepsilon}^{\mathbb{N}},
$$

which is in turn equal to

$$
\int_{M} \widetilde{\mathcal{P}}_{\varepsilon, m}^{m} h_{\varepsilon}(x) \mathrm{dLeb}
$$

where we have now defined

$$
\widetilde{\mathcal{P}}_{\varepsilon, m} \psi(x):=\mathcal{P}_{\varepsilon}\left(\mathbf{1}_{U_{m}^{c}} \psi\right)(x) .
$$

Let us note that the operator $\widetilde{\mathcal{P}}_{\varepsilon, m}$ depends on $m$ via the set $U_{m}$, and not on $\varepsilon$ which is kept fixed, and that $\widetilde{\mathcal{P}}_{\varepsilon, m}$ "reduces" to $\mathcal{P}_{\varepsilon}$ as $m \rightarrow \infty$. It is therefore tempting to consider $\widetilde{\mathcal{P}}_{\varepsilon, m}$ as a small perturbation of $\mathcal{P}_{\varepsilon}$ when $m$ is large and to check if it shares the spectral properties of a REPFO operator. We will show in a moment that it will be the case; let us now see what that implies for our theory. 
5.2. Limiting distributions. We now indicate the correspondences between the general notation of Keller's results and our own quantities:

$$
\begin{gathered}
P_{0} \Rightarrow \mathcal{P}_{\varepsilon}, \\
P_{\varepsilon} \Rightarrow \widetilde{\mathcal{P}}_{\varepsilon, m} ; Q_{\varepsilon} \Rightarrow Q_{\varepsilon, m}, \\
\varphi_{\varepsilon} \Rightarrow \varphi_{\varepsilon, m} ; \varphi_{0} \Rightarrow h_{\varepsilon}, \\
\lambda_{\varepsilon} \Rightarrow \lambda_{\varepsilon, m}, \\
\nu_{\varepsilon} \Rightarrow \nu_{\varepsilon, m} ; \nu_{0} \Rightarrow \operatorname{Leb}, \\
\Delta_{\varepsilon} \Rightarrow \Delta_{\varepsilon, m}=\mu_{\varepsilon}\left(U_{m}\right)=\operatorname{Leb}\left(\left(\mathcal{P}_{\varepsilon}-\widetilde{\mathcal{P}}_{\varepsilon, m}\right) h_{\varepsilon}\right) .
\end{gathered}
$$

The framework for which we will prove the assumptions (A1)-(A4) for our REPFO $\widetilde{\mathcal{P}}_{\varepsilon, m}$ are those behind the system and its perturbations which we introduced in the previous sections and which we summarize here:

Hypotheses on the system and its perturbations. We consider piecewise expanding maps $f$ of the circle or of the interval $I$ which verify:

H1 The map $f$ admits a (unique) a.c.i.p. which is mixing.

H2 We will require that

$$
\inf _{x \in I}|D f(x)| \geq \beta>1
$$

and

$$
\sup _{x \in I}\left|\frac{D^{2} f(x)}{D f(x)^{2}}\right| \leq C_{1}<\infty
$$

whenever the first and the second derivatives are defined.

H3 The couple of adapted spaces upon which the REPFO operators will act are: the space of functions of bounded variation (as in Definition 3.1] we will indicate with Var the total variation), and $L^{1}(\mathrm{Leb})$, with norm $\|\cdot\|_{1}$. This time, we will write $\|\cdot\|_{B V}=\operatorname{Var}(\cdot)+\|\cdot\|_{1}$ for the associated Banach norm.

H4 There exists a monotone upper semi-continuous function $p: \Omega \rightarrow[0, \infty)$ such that $\lim _{\varepsilon \rightarrow 0} p_{\varepsilon}=0$ and $\forall f \in \mathrm{BV}, \forall \varepsilon \in \Omega:\left\|\mathcal{P} f-\mathcal{P}_{\varepsilon} f\right\|_{1} \leq p_{\varepsilon}\|f\|_{B V} \overrightarrow{4}$

H5 The density $h_{\varepsilon}$ of the stationary measure is bounded from below Leb-a.e. and we call this bound $\underline{h}_{\varepsilon}$.

Extreme values. Let us therefore write $\widetilde{\mathcal{P}}_{\varepsilon, m} \varphi_{\varepsilon, m}=\lambda_{\varepsilon, m} \varphi_{\varepsilon, m}, \nu_{\varepsilon, m} \widetilde{\mathcal{P}}_{\varepsilon, m}=$ $\lambda_{\varepsilon, m} \nu_{\varepsilon, m}$, and $\lambda_{\varepsilon, m}^{-1} \widetilde{\mathcal{P}}_{\varepsilon, m}=\varphi_{\varepsilon, m} \otimes \nu_{\varepsilon, m}+Q_{\varepsilon, m}$.

\footnotetext{
${ }^{4}$ This condition can be checked in several cases. We did it, for instance, in the previous section in 4.2.2. A general theorem is presented in Lemma 16 in 19 for piecewise expanding maps of the interval endowed with our pair of adapted spaces and with the noise given by a convolution kernel. This means that $\theta_{\varepsilon}$ is absolutely continuous with respect to Lebesgue on the space $\Omega$ with density $s_{\varepsilon}$, and our two operators are related by the convolution formula $\mathcal{P}_{\varepsilon} g(x)=\int_{\Omega}(\mathcal{P} g)(x-\omega) s_{\varepsilon}(\omega) \mathrm{d} \omega$, where $g \in B V$. In the case of additive noise, it is straightforward to check that the previous formula is equivalent to $\mathcal{P}_{\varepsilon} g(x)=\int_{\Omega}\left(\mathcal{P}_{\omega} g\right)(x) s_{\varepsilon}(\omega) \mathrm{d} \omega$, where $\mathcal{P}_{\omega}$ is the Perron-Frobenius operator of the transformation $f_{\omega}$.
} 
Then formula (5.7) implies that $1-\lambda_{\varepsilon, m}=\Delta_{\varepsilon, m} \vartheta_{\varepsilon}(1+o(1))$. We can therefore write

$$
\begin{aligned}
\left(\mu_{\varepsilon}\right. & \left.\times \theta_{\varepsilon}^{\mathbb{N}}\right)\left(M_{m} \leq u_{m}\right) \\
& =\int_{M} \widetilde{\mathcal{P}}_{\varepsilon, m}^{m} h_{\varepsilon}(x) \mathrm{dLeb}=\lambda_{\varepsilon, m}^{m} \int h_{\varepsilon} \mathrm{d} \nu_{\varepsilon, m}+\lambda_{\varepsilon, m}^{m} \int Q_{\varepsilon, m} h_{\varepsilon} \mathrm{dLeb} \\
& =e^{-\left(\vartheta_{\varepsilon} m \mu_{\varepsilon}\left(U_{m}\right)+m o\left(\mu_{\varepsilon}\left(U_{m}\right)\right)\right)} \int h_{\varepsilon} \mathrm{d} \nu_{\varepsilon, m}+\mathcal{O}\left(\lambda_{\varepsilon, m}^{m}\left\|Q_{\varepsilon, m}\right\|_{B V}\right) .
\end{aligned}
$$

Remember that we are under the assumption that

$$
m\left(\mu_{\varepsilon} \times \theta_{\varepsilon}^{\mathbb{N}}\right)\left(\phi>u_{m}\right)=m \mu_{\varepsilon}\left(\phi>u_{m}\right)=m \mu_{\varepsilon}\left(U_{m}\right) \rightarrow \tau,
$$

when $m \rightarrow \infty$; moreover, it follows from the theory of [21] that $\int h_{\varepsilon} \mathrm{d} \nu_{\varepsilon, m} \rightarrow$ $\int h_{\varepsilon} \mathrm{dLeb}=1$, as $m$ goes to infinity. In conclusion we get

$$
\left(\mu_{\varepsilon} \times \theta_{\varepsilon}^{\mathbb{N}}\right)\left(M_{m} \leq u_{m}\right)=e^{-\tau \vartheta_{\varepsilon}}(1+o(1))
$$

in the limit $m \rightarrow \infty$ and where $\vartheta_{\varepsilon}$ will be the extremal index, and this will be explicitly computed later on for some particular maps thanks to formula (5.9) and shown to be equal to 1 for any point $\zeta$; see Proposition 5.3 below.

Random hitting times. Let us denote again with $r \frac{\omega}{U_{m}}(x)$ the first entrance into the ball $U_{m}$. A direct application of [20, Proposition 2], and which is true for REPFO operators, allows us to get the following result, which we adapted to our situation and which provides an explicit formula for the statistics of the first hitting times in the annealed case. Notice that this result strengthens our Corollary E since it provides the error in the convergence to the exponential law.

Proposition 5.1. For the REPFO $\widetilde{\mathcal{P}}_{\varepsilon, m}$ which verifies the hypotheses $\mathbf{H 1 - H 5}$, and using the notation introduced above, there exists a constant $C>0$ such that for all $m$ big enough there exists $\xi_{m}>0$ s.t. for all $t>0$,

$$
\left|\left(\mu_{\varepsilon} \times \theta_{\varepsilon}^{\mathbb{N}}\right)\left\{r \frac{\omega}{U_{m}}>\frac{t}{\xi_{m} \mu_{\varepsilon}\left(U_{m}\right)}\right\}-e^{-t}\right| \leq C \delta_{m}(t \vee 1) e^{-t},
$$

where $\delta_{m}=O\left(\eta_{m} \log \eta_{m}\right)$,

$$
\eta_{m}:=\sup \left\{\left|\int_{U_{m}} \psi \mathrm{dLeb}\right| ;\|\psi\|_{B V} \leq 1\right\}=\operatorname{Leb}\left(U_{m}\right)
$$

and $\xi_{m}$ goes to $\vartheta_{\varepsilon}$ as $m \rightarrow \infty$.

\subsection{Cheking assumptions (A1)-(A4).}

Proposition 5.2. For the REPFO $\widetilde{\mathcal{P}}_{\varepsilon, m}$ which verifies the hypotheses $\mathbf{H 1}-\mathbf{H} 5$, the assumptions (A1)-(A4) hold.

Proof. Condition (A1) means to prove the Lasota-Yorke inequality for the operator $\widetilde{\mathcal{P}}_{\varepsilon, m}$. We recall that the constants $A$ and $B$ there must independent of the perturbation parameter which in our case is $m$. We begin with the total variation.

The structure of $\widetilde{\mathcal{P}}_{\varepsilon, m}$ 's iterates is

$$
\left(\widetilde{\mathcal{P}}_{\varepsilon, m}^{n} \psi\right)=\int \cdots \int \mathcal{P}_{\omega_{n}}\left(\mathbf{1}_{U_{m}^{c}} \mathcal{P}_{\omega_{n-1}}\left(\mathbf{1}_{U_{m}^{c}} \cdots \mathcal{P}_{\omega_{1}}\left(\psi \mathbf{1}_{U_{m}^{c}}\right)\right)\right) \mathrm{d} \theta_{\varepsilon}\left(\omega_{1}\right) \cdots \mathrm{d} \theta_{\varepsilon}\left(\omega_{n}\right) .
$$


Let us call $A_{l, \omega_{j}}$ the $l$-domain of injectivity of the map $f_{\omega_{j}}$ and call $f_{l, \omega_{j}}^{-1}$ the inverse of $f_{\omega_{j}}$ restricted to $A_{l, \omega_{j}}$. We have:

$$
\begin{aligned}
& \Upsilon_{\omega_{1}, \ldots, \omega_{n}}:=\mathcal{P}_{\omega_{n}}\left(\mathbf{1}_{U_{m}^{c}} \mathcal{P}_{\omega_{n-1}}\left(\mathbf{1}_{U_{m}^{c}} \cdots \mathcal{P}_{\omega_{1}}\left(\psi \mathbf{1}_{U_{m}^{c}}\right)\right)\right)(x) \\
&=\sum_{k_{n}, \ldots, k_{1}} \frac{\left(\psi \cdot \mathbf{1}_{U_{m}^{c}} \cdot \mathbf{1}_{U_{m}^{c}} \circ f_{\omega_{1}} \cdots \mathbf{1}_{U_{m}^{c}} \circ f_{\omega_{n-1}} \circ \cdots \circ f_{\omega_{1}}\right)\left(\left(f_{k_{1}, \omega_{1}}^{-1} \circ \cdots \circ f_{k_{n}, \omega_{n}}^{-1}\right)(x)\right)}{\left|D\left(f_{\omega_{n}} \circ \cdots \circ f_{\omega_{1}}\right)\left(\left(f_{k_{1}, \omega_{1}}^{-1} \circ \cdots \circ f_{k_{n}, \omega_{n}}^{-1}\right)(x)\right)\right|} \\
& \times \mathbf{1}_{f_{\omega_{n}} \circ \cdots \circ f_{\omega_{1}}} \Omega_{\omega_{1}, \ldots, \omega_{n}}^{k_{1}, \ldots, k_{n}}(x) .
\end{aligned}
$$

The sets

$$
\begin{array}{r}
\Omega_{\omega_{1}, \ldots, \omega_{n}}^{k_{1}, \ldots, k_{n}}:=f_{k_{1}, \omega_{1}}^{-1} \circ \cdots \circ f_{k_{n-1}, \omega_{n-1}}^{-1} A_{k_{n}, \omega_{n}} \cap f_{k_{1}, \omega_{1}}^{-1} \circ \cdots \circ f_{k_{n-2}, \omega_{n-2}}^{-1} A_{k_{n-1}, \omega_{n-1}} \\
\cap \cdots \cap f_{k_{1}, \omega_{1}}^{-1} A_{k_{2}, \omega_{2}} \cap A_{k_{1}, \omega_{1}}
\end{array}
$$

are intervals and they give a mod-0 partition of $I=[0,1]$; moreover, the image $H_{\omega_{1}, \ldots, \omega_{n}}^{k_{1}, \ldots, k_{n}}:=f_{\omega_{n}} \circ \cdots \circ f_{\omega_{1}} \Omega_{\omega_{1}, \ldots, \omega_{n}}^{k_{1}, \ldots, k_{n}}$ for a given $n$-tuple $\left\{k_{n}, \ldots, k_{1}\right\}$ is a connected interval. We also note for future purposes that we could equivalently write:

$$
g_{n}:=\mathbf{1}_{U_{m}^{c}} \cdot \mathbf{1}_{U_{m}^{c}} \circ f_{\omega_{1}} \cdot \ldots \cdot \mathbf{1}_{U_{m}^{c}} f_{\omega_{n-1}} \circ \cdots \circ f_{\omega_{1}}=\mathbf{1}_{U_{m}^{c} \cap f_{\omega_{1}}^{-1} U_{m}^{c} \cap \cdots \cap f_{\omega_{1}}^{-1} \circ \cdots \circ f_{\omega_{n-1}}^{-1} U_{m}^{c}} .
$$

Now we observe that the set

$$
U_{m}^{c}(n):=U_{m}^{c} \cap f_{\omega_{1}}^{-1} U_{m}^{c} \cap f_{\omega_{1}}^{-1} \circ f_{\omega_{2}}^{-1} U_{m}^{c} \cap \cdots \cap f_{\omega_{1}}^{-1} \circ \cdots \circ f_{\omega_{n-1}}^{-1} U_{m}^{c} \cap \Omega_{\omega_{1}, \ldots, \omega_{n}}^{k_{1}, \ldots, k_{n}}
$$

is actually given by

$U_{m}^{c}(n):=U_{m}^{c} \cap f_{k_{1}, \omega_{1}}^{-1} U_{m}^{c} \cap f_{k_{1}, \omega_{1}}^{-1} \circ f_{k_{2}, \omega_{2}}^{-1} U_{m}^{c} \cap \cdots \cap f_{k_{1}, \omega_{1}}^{-1} \circ \cdots \circ f_{k_{n-1}, \omega_{n-1}}^{-1} U_{m}^{c} \cap \Omega_{\omega_{1}, \ldots, \omega_{n}}^{k_{1}, \ldots, k_{n}}$.

Since $U_{m}^{c}$ is the disjoint union of two connected intervals, the number of connected intervals in $U_{m}^{c}(n)$ is bounded from above by $n+1$ and it is important that it grows linearly with $n$. We now take the total variation $\operatorname{Var}\left(\Upsilon_{\omega_{1}, \ldots, \omega_{n}}\right)$. We begin to remark that, by standard techniques,

$$
\begin{gathered}
\operatorname{Var}\left(\frac{\left(\psi g_{n}\right)\left(\left(f_{k_{1}, \omega_{1}}^{-1} \circ \cdots \circ f_{k_{n}, \omega_{n}}^{-1}\right)(x)\right)}{\left|D\left(f_{\omega_{n}} \circ \cdots \circ f_{\omega_{1}}\right)\left(\left(f_{k_{1}, \omega_{1}}^{-1} \circ \cdots \circ f_{k_{n}, \omega_{n}}^{-1}\right)(x)\right)\right|} \mathbf{1}_{f_{\omega_{n}} \circ \cdots \circ f_{\omega_{1}}} \Omega_{\omega_{1}, \ldots, \omega_{n}}^{k_{1}, \ldots, k_{n}}(x)\right) \\
\leq 2 \operatorname{Var}_{H_{\omega_{1}, \ldots, \omega_{n}}^{k_{1}, \ldots, k_{n}}}\left(\frac{\left(\psi g_{n}\right)\left(\left(f_{k_{1}, \omega_{1}}^{-1} \circ \cdots \circ f_{k_{n}, \omega_{n}}^{-1}\right)(x)\right)}{\left|D\left(f_{\omega_{n}} \circ \cdots \circ f_{\omega_{1}}\right)\left(\left(f_{k_{1}, \omega_{1}}^{-1} \circ \cdots \circ f_{k_{n}, \omega_{n}}^{-1}\right)(x)\right)\right|}\right) \\
+\frac{2}{\beta^{n}} \frac{1}{\operatorname{Leb}\left(\Omega_{\omega_{1}, \ldots, \omega_{n}}^{k_{1}, \ldots, k_{n}}\right)} \int_{\Omega_{\omega_{1}, \ldots, \omega_{n}}^{k_{1}, \ldots, k_{n}}}\left|\psi g_{n}\right| \mathrm{dLeb},
\end{gathered}
$$

where $\beta$ is given by (5.18) in $\mathbf{H 2}$.

The variation above can be further estimated by standard techniques:

$$
\begin{array}{r}
\leq \frac{2}{\beta^{n}} \operatorname{Var}_{\Omega_{\omega_{1}, \ldots, \omega_{n}}^{k_{1}, \ldots, k_{n}}}\left(\psi g_{n}\right)+\frac{2}{\beta^{n}} \frac{1}{\operatorname{Leb}\left(\Omega_{\omega_{1}, \ldots, \omega_{n}}^{k_{1}, \ldots, k_{n}}\right)} \int_{\Omega_{\omega_{1}, \ldots, \omega_{n}}^{k_{1}, \ldots, k_{n}}}\left|\psi g_{n}\right| \mathrm{dLeb} \\
+2 \sup _{\zeta, \omega_{1}, \ldots, \omega_{n}} \frac{\left|D^{2}\left(f_{\omega_{n}} \circ \cdots \circ f_{\omega_{1}}\right)(\zeta)\right|}{\left[D\left(f_{\omega_{n}} \circ \cdots \circ f_{\omega_{1}}\right)(\zeta)\right]^{2}} \int_{\Omega_{\Omega_{1}, \ldots, \omega_{n}}^{k_{1}, \ldots, k_{n}}}\left|\psi g_{n}\right| \text { dLeb. }
\end{array}
$$


We now have:

$$
\begin{aligned}
& \frac{\left|D^{2}\left(f_{\omega_{n}} \circ \cdots \circ f_{\omega_{1}}\right)(\zeta)\right|}{\left[D\left(f_{\omega_{n}} \circ \cdots \circ f_{\omega_{1}}\right)(\zeta)\right]^{2}} \\
& \quad=\sum_{k=0}^{n-1} \frac{D^{2} f_{\omega_{n-k}}\left(\prod_{l=1}^{n-1-k} T_{\omega_{n-l}}(\zeta)\right)}{\left[D f_{\omega_{n-k}}\left(\prod_{l=1}^{n-1-k} f_{\omega_{n-l}}(\zeta)\right)\right]^{2} \prod_{j=0}^{k} D f_{\omega_{n-j+1}}\left(\prod_{l=1}^{n-j} f_{\omega_{n-l}}(\zeta)\right)} .
\end{aligned}
$$

By (5.19) in $\mathbf{H 2}$ and again using (5.18), the previous sum will be bounded by $C_{1}$ times the sum of a geometric series of reason $\beta^{-1}$; we call $C$ the upper bound thus found. Our variation above is therefore bounded by

$$
\begin{aligned}
\text { (5.21) } \leq & \frac{2}{\beta^{n}} \operatorname{Var}_{\Omega_{\omega_{1}, \ldots, \omega_{n}}^{k_{1}, \ldots, k_{n}}}\left(\psi g_{n}\right)+\frac{2}{\beta^{n}} \frac{1}{\operatorname{Leb}\left(\Omega_{\omega_{1}, \ldots, \omega_{n}}^{k_{1}, \ldots, k_{n}}\right)} \int_{\Omega_{\omega_{1}, \ldots, \omega_{n}}^{k_{1}, \ldots, k_{n}}}\left|\psi g_{n}\right| \mathrm{dLeb} \\
& +2 C \int_{\Omega_{\omega_{1}, \ldots, \omega_{n}}^{k_{1}, \ldots, \omega_{n}}}\left|\psi g_{n}\right| \text { dLeb. }
\end{aligned}
$$

Now:

$$
\begin{aligned}
\operatorname{Var}_{\Omega_{\omega_{1}, \ldots, \omega_{n}}^{k_{1}, \ldots, k_{n}}}\left(\psi g_{n}\right) \leq & \operatorname{Var}_{\Omega_{\omega_{1}, \ldots, \omega_{n}}^{k_{1}, \ldots, k_{n}}}(\psi)+2(n+1) \sup _{\substack{\Omega_{\omega_{1}, \ldots, \omega_{n}}^{k_{1}, \ldots, k_{n}} \\
\leq}}|\psi| \\
\leq & {[2(n+1)+1] \operatorname{Var}_{\Omega_{\omega_{1}, \ldots, \omega_{n}}^{k_{1}, \ldots, k_{n}}}(\psi) } \\
& +\frac{1}{\operatorname{Leb}\left(\Omega_{\omega_{1}, \ldots, \omega_{n}}^{k_{1}, \ldots, k_{n}}\right)} \int_{\Omega_{\omega_{1}, \ldots, \omega_{n}}^{k_{1}, \ldots, k_{n}}}|\psi| \mathrm{dLeb},
\end{aligned}
$$

where $2(n+1)$ is an estimate from above of the number of jumps of $g_{n}$. We now observe that for a finite realisation of length $n, \omega_{1}, \ldots, \omega_{n}$, the quantity $\Psi_{n, \omega_{1}, \ldots, \omega_{n}}=\inf _{k_{1}, \ldots, k_{n}} \operatorname{Leb}\left(\Omega_{\omega_{1}, \ldots, \omega_{n}}^{k_{1}, \ldots, k_{n}}\right)$, where each $k_{j}$ runs over the finite branches of $f_{\omega_{j}}$, is surely strictly positive and also implies that $\Psi_{n}^{-1}:=\int \Psi_{n, \omega_{1}, \ldots, \omega_{n}}^{-1} \mathrm{~d} \theta_{\varepsilon}^{\mathbb{N}}>0$. We now replace (5.23) into (5.22), we sum over the $k_{1}, \ldots, k_{n}$ and we integrate w.r.t. $\theta_{\varepsilon}^{\mathbb{N}}$; finally we get

$$
\operatorname{Var}\left(\widetilde{\mathcal{P}}_{\varepsilon, m}^{n} \psi\right) \leq \frac{2}{\beta^{n}}(2 n+3) \operatorname{Var}(\psi)+\left[\frac{4}{\beta^{n}} \frac{1}{\Psi_{n}}+2 C\right] \int_{I}|\psi| \mathrm{dLeb} .
$$

In order to get the Lasota-Yorke inequality one should get a certain $n_{0}$ and a number $\beta>\kappa>1$ and such that

$$
\frac{2}{\beta^{n_{0}}}\left(2 n_{0}+3\right)<\kappa^{-n_{0}}
$$

the Lasota-Yorke inequality (5.10) will then follow with standard arguments 5

We now compute the $L^{1}$-norm of our operator. We have to compute $\left\|\widetilde{\mathcal{P}}_{\varepsilon, m}^{n} \psi\right\|_{1}$; by splitting $\psi$ into the sum of its positive and negative parts and by using the linearity of the transfer operator, we may suppose that $\psi$ is non-negative. This

\footnotetext{
${ }^{5}$ By defining $A=2\left(2 n_{0}+3\right)$ and $B=\left[\frac{4}{\Psi_{n_{0}}}+2 C\right] \frac{2}{1-\kappa^{-n_{0}}}$, we have

$$
\operatorname{Var}\left(\widetilde{\mathcal{P}}_{\varepsilon, m}^{n} \psi\right) \leq A \kappa^{-n} \operatorname{Var}(\psi)+B \int_{I}|\psi| \text { dLeb. }
$$
}


allows us to interchange the integrals w.r.t. the Lebesgue measure and $\theta_{\varepsilon}^{\mathbb{N}}$ and to use duality for each of the $\mathcal{P}_{\omega}$. In conclusion we get

$$
\left\|\widetilde{\mathcal{P}}_{\varepsilon, m}^{n} \psi\right\|_{1} \leq \int|\psi| h_{\varepsilon} \mathbf{1}_{U_{m}^{c}}(x) \mathbf{1}_{U_{m}^{c}}\left(f_{\omega_{1}} x\right) \cdots \mathbf{1}_{U_{m}^{c}}\left(f_{\omega_{n-1}} \circ \cdots \circ f_{\omega_{1}} x\right) \mathrm{dLeb} \leq\|\psi\|_{1} .
$$

This concludes the proof of the Lasota-Yorke inequality, (A1). We now have to show that the operator $\mathcal{P}_{\varepsilon}$, which is the unperturbed operator w.r.t. $\widetilde{\mathcal{P}}_{\varepsilon, m}$, verifies the mixing condition (A2). Now the Perron-Frobenius operator $\mathcal{P}$ for the original map $f$, which is in turn the unperturbed operator w.r.t. $\mathcal{P}_{\varepsilon}$, is mixing ( 1 is the only eigenvalue of finite multiplicity on the unit circle), since our original map $f$ was chosen to be mixing (hypothesis H1), and therefore, by the perturbation theory in [21] and the closeness of the two operators expressed by assumption $\mathbf{H} \mathbf{4}, \mathcal{P}_{\varepsilon}$ is also a mixing operator. Let us discuss the assumption (A3).

Let us bound the following quantity, for any $\psi$ of bounded variation and of total variation less than or equal to 1 :

$$
\begin{aligned}
\left|\int_{I}\left(\widetilde{\mathcal{P}}_{\varepsilon, m} \psi(x)-\mathcal{P}_{\varepsilon} \psi(x)\right) \mathrm{d} \operatorname{Leb}(x)\right|=\left|\int_{I} \mathcal{P}_{\varepsilon}\left(\mathbf{1}_{U_{m}} \psi\right)(x) \mathrm{d} \operatorname{Leb}(x)\right| \\
\leq\left|\int\left(\int_{I} \mathcal{P}_{\omega}\left(\mathbf{1}_{U_{m}} \psi\right) \mathrm{dLeb}\right) \mathrm{d} \theta_{\varepsilon}(\omega)\right| \leq\|\psi\|_{\infty} \operatorname{Leb}\left(U_{m}\right),
\end{aligned}
$$

where $\|\psi\|_{\infty} \leq\|\psi\|_{B V}$ and $\operatorname{Leb}\left(U_{m}\right)$ goes to zero when $m$ goes to infinity.

We now check assumption (A4) under the hypothesis $\mathbf{H 5}$.

We have:

$$
\left\|\left(\widetilde{\mathcal{P}}_{\varepsilon, m}-\mathcal{P}_{\varepsilon}\right) h_{\varepsilon}\right\|_{B V}=\left\|\mathcal{P}_{\varepsilon}\left(\mathbf{1}_{U_{m}} h_{\varepsilon}\right)\right\|_{B V} \leq A \kappa^{-1}\left\|\mathbf{1}_{U_{m}} h_{\varepsilon}\right\|_{B V}+B\left\|\mathbf{1}_{U_{m}} h_{\varepsilon}\right\|_{1} .
$$

The right hand side is bounded by a constant $C^{*}$ which is independent of $m$. We recall that in our case $\Delta_{\varepsilon, m}=\mu_{\varepsilon}\left(U_{m}\right)$ and that

$$
\eta_{\varepsilon, m}:=\sup _{\|\psi\|_{B V} \leq 1}\left|\int_{I}\left(\widetilde{\mathcal{P}}_{\varepsilon, m} \psi(x)-\mathcal{P}_{\varepsilon} \psi(x)\right) \operatorname{dLeb}(x)\right| \leq \operatorname{Leb}\left(U_{m}\right)
$$

(see the computation above). Then

$$
\left\|\left(\widetilde{\mathcal{P}}_{\varepsilon, m}-\mathcal{P}_{\varepsilon}\right) h_{\varepsilon}\right\|_{B V} \leq C^{*} \frac{\mu_{\varepsilon}\left(U_{m}\right)}{\underline{h}_{\varepsilon} \operatorname{Leb}\left(U_{m}\right)} \leq C^{*} \frac{\Delta_{\varepsilon, m}}{\eta_{\varepsilon, m}} .
$$

5.4. Extremal index. In this part, we investigate the quantity (see (5.8) and (5.9) ):

$$
q_{k, m}=\frac{\operatorname{Leb}\left(\left(\mathcal{P}_{\varepsilon}-\widetilde{\mathcal{P}}_{\varepsilon, m}\right) \widetilde{\mathcal{P}}_{\varepsilon, m}^{k}\left(\mathcal{P}_{\varepsilon}-\widetilde{\mathcal{P}}_{\varepsilon, m}\right)\left(h_{\varepsilon}\right)\right)}{\mu_{\varepsilon}\left(U_{m}\right)} .
$$

We recall that $U_{m}:=U_{m}(\zeta)$ represents a ball around the point $\zeta$. Our result is the following.

Proposition 5.3. Let us suppose that $f$ is either a $C^{2}$ expanding map of the circle or a piecewise expanding map of the circle with finite branches and verifying hypotheses H1-H4. Then for each $k$,

$$
\lim _{m \rightarrow \infty} q_{k, m} \equiv 0
$$


i.e., the limit in the definition of $q_{k}$ in (5.8) exists and equals zero. Also the extremal index verifies $\vartheta=1-\sum_{k=0}^{\infty} q_{k}=1$, and this is independent of the point $\zeta$, the center of the ball $U_{m}$.

Proof. Let us define $G_{k, m} \equiv \int\left(\mathcal{P}_{\varepsilon}-\widetilde{\mathcal{P}}_{\varepsilon, m}\right) \widetilde{\mathcal{P}}_{\varepsilon, m}^{k}\left(\mathcal{P}_{\varepsilon}-\widetilde{\mathcal{P}}_{\varepsilon, m}\right) h_{\varepsilon}$ dLeb.

As $\left(\mathcal{P}_{\varepsilon}-\widetilde{\mathcal{P}}_{\varepsilon, m}\right) \psi=\mathcal{P}_{\varepsilon}\left(\mathbf{1}_{U_{m}} \psi\right)$, we may write

$$
G_{k, m}=\int \mathbf{1}_{U_{m}}(x) \widetilde{\mathcal{P}}_{\varepsilon, m}^{k}\left(\mathcal{P}_{\varepsilon}-\widetilde{\mathcal{P}}_{\varepsilon, m}\right) h_{\varepsilon} \text { dLeb. }
$$

By using (5.20) we get

$$
\begin{aligned}
G_{k, m}= & \iint \mathbf{1}_{U_{m}}\left(f_{\omega_{k+1}} \circ f_{\omega_{k}} \circ \cdots \circ f_{\omega_{1}} x\right) \mathbf{1}_{U_{m}^{c}}\left(f_{\omega_{k}} \circ \cdots \circ f_{\omega_{1}} x\right) \\
& \cdots \mathbf{1}_{U_{m}^{c}}\left(f_{\omega_{1}} x\right) \mathbf{1}_{U_{m}}(x) h_{\varepsilon}(x) \mathrm{dLebd} \theta_{\varepsilon}^{\mathbb{N}} .
\end{aligned}
$$

In order to simplify the notation let us put

$\psi_{k, U_{m}, \underline{\omega}}(x)=\mathbf{1}_{U_{m}}\left(f_{\omega_{k+1}} \circ f_{\omega_{k}} \circ \cdots \circ f_{\omega_{1}} x\right) \mathbf{1}_{U_{m}^{c}}\left(f_{\omega_{k}} \circ \cdots \circ f_{\omega_{1}} x\right) \ldots \mathbf{1}_{U_{m}^{c}}\left(f_{\omega_{1}} x\right) \mathbf{1}_{U_{m}}(x)$.

Now let us prove that $q_{k, m}$ converges to 0 . Our approach is very similar to what we did to prove $D^{\prime}\left(u_{m}\right)$, and we now split the proof according to the regularity of the map.

(i) Suppose that $f: \mathcal{S}^{1} \rightarrow \mathcal{S}^{1}$ is a $C^{2}$, expanding map, i.e., there exists $|D f(x)|>$ $\lambda>1$, for all $x \in \mathcal{S}^{1}$. First, note that since $S^{1}$ is compact and $f$ is $C^{2}$, there exists $\sigma>1$ such that $|D f(x)| \leq \sigma$. Hence the set $U_{m}$ grows at most at a rate given by $\sigma$, so, for any $\underline{\omega} \in \Omega^{\mathbb{N}}$ we have $\left|f_{\underline{\omega}}^{j}\left(U_{m}\right)\right| \leq \sigma^{j}\left|U_{m}\right|$. This implies that

$$
\text { if } \operatorname{dist}\left(f_{\underline{\omega}}^{j}(\zeta), \zeta\right)>2 \sigma^{j}\left|U_{m}\right|>\left|U_{m}\right|+\sigma^{j}\left|U_{m}\right| \text {, then } f_{\underline{\omega}}^{j}\left(U_{m}\right) \cap U_{m}=\emptyset \text {. }
$$

Note that, by inequality (5.25), if for all $j=1, \ldots, k+1$ we have $\operatorname{dist}\left(f_{\underline{\omega}}^{j}(\zeta), \zeta\right)>$ $2 \sigma^{j}\left|U_{m}\right|$, then clearly $\psi_{k, B_{m}, \underline{\omega}}(x)=0$, for all $x$. We define

$$
W_{k, m}=\bigcap_{j=1}^{k+1}\left\{\underline{\omega} \in(-\varepsilon, \varepsilon)^{\mathbb{N}}: \operatorname{dist}\left(f_{\underline{\omega}}^{j}(\zeta), \zeta\right)>2 \sigma^{j}\left|U_{m}\right|\right\} .
$$

Note that on $W_{k, m}$ we have $\psi_{k, U_{m}, \underline{\omega}}=0$. We want to compute the measure of $W_{k, m}^{c}$.

Observe that $W_{k, m}^{c} \subset \bigcup_{j=1}^{k+1}\left\{\underline{\omega}: f_{\underline{\omega}}^{j}(\zeta) \in B_{2 \sigma^{j}\left|U_{m}\right|}(\zeta)\right\}$. Hence, we have

$$
\begin{aligned}
\theta_{\varepsilon}^{\mathbb{N}}\left(W_{k, m}^{c}\right) & \leq \sum_{j=1}^{k+1} \int \theta_{\varepsilon}\left(\left\{\omega_{j}: f\left(f_{\underline{\omega}}^{j-1}(\zeta)\right)+\omega_{j} \in B_{2 \sigma^{j}\left|U_{m}\right|}(\zeta)\right\}\right) \mathrm{d} \theta_{\varepsilon}^{\mathbb{N}} \\
& \leq \sum_{j=1}^{k+1} \overline{g_{\varepsilon}}\left|B_{2 \sigma^{j}\left|U_{m}\right|}(\zeta)\right|=\sum_{j=1}^{k+1} \overline{g_{\varepsilon}} 4 \sigma^{j}\left|U_{m}\right| \leq 4 \overline{g_{\varepsilon}}\left|U_{m}\right| \frac{\sigma}{\sigma-1} \sigma^{k+1} .
\end{aligned}
$$

Using this estimate we obtain:

$$
\begin{aligned}
G_{k, m} & =\int_{W_{k, m}} \int \psi_{k, U_{m}, \underline{\omega}}(x) h_{\varepsilon}(x) \mathrm{d} \operatorname{Leb} \mathrm{d} \theta_{\varepsilon}^{\mathbb{N}}+\int_{W_{k, m}^{c}} \int \psi_{k, U_{m}, \underline{\omega}}(x) h_{\varepsilon}(x) \mathrm{d} \operatorname{Leb} \mathrm{d} \theta_{\varepsilon}^{\mathbb{N}} \\
& =0+\int_{W_{k, m}^{c}} \int \psi_{k, U_{m}, \underline{\omega}}(x) h_{\varepsilon}(x) \mathrm{dLebd} \theta_{\varepsilon}^{\mathbb{N}}
\end{aligned}
$$


and because $\psi_{k, U_{m}, \underline{\omega}}(x) \leq \mathbf{1}_{U_{m}}(x)$, we have:

$$
\begin{aligned}
& \leq \int_{W_{k, m}^{c}} \int \mathbf{1}_{U_{m}}(x) h_{\varepsilon}(x) \mathrm{d} \operatorname{Leb} \mathrm{d} \theta_{\varepsilon}^{\mathbb{N}} \leq \mu_{\varepsilon}\left(U_{m}\right) \theta_{\varepsilon}^{\mathbb{N}}\left(W_{k, m}^{c}\right) \\
& \leq \mu_{\varepsilon}\left(U_{m}\right) 4 \overline{g_{\varepsilon}}\left|U_{m}\right| \frac{\sigma}{\sigma-1} \sigma^{k+1} .
\end{aligned}
$$

Now recall that $q_{k, m}=\frac{G_{k, m}}{\mu_{\varepsilon}\left(U_{m}\right)}$. It follows that

$$
q_{k, m} \leq \frac{\mu_{\varepsilon}\left(U_{m}\right) 4 \overline{g_{\varepsilon}}\left|U_{m}\right| \frac{\sigma}{\sigma-1} \sigma^{k+1}}{\mu_{\varepsilon}\left(U_{m}\right)} \leq 4 \overline{g_{\varepsilon}}\left|U_{m}\right| \frac{\sigma}{\sigma-1} \sigma^{k+1} \underset{m \rightarrow \infty}{\longrightarrow} 0 .
$$

(ii) Using the same ideas as in the previous section, we can extend this result to the piecewise expanding maps with finite branches. Recall that we need to define some 'safety boxes' in order to use the same arguments as in the continuous case. So, if for all $j=1, \ldots, k+1$ and $i=1, \ldots, \ell$, where $\ell$ stands for the number of discontinuity points, we have

$$
\operatorname{dist}\left(f_{\underline{\omega}}^{j}(\zeta), \xi_{i}\right)>2 \sigma^{j}\left|U_{m}\right|,
$$

then the set $U_{m}$ consists of one connected component at each iteration, and also we have $f_{\underline{\omega}}^{j}\left(U_{m}\right) \cap U_{m}=\emptyset$ which means $\psi_{k, U_{m}, \underline{\omega}}(x)=0$, for all $x$. Now let us define

$$
W_{k, m}=\bigcap_{j=1}^{k+1} \bigcap_{i=0}^{\ell}\left\{\underline{\omega} \in(-\varepsilon, \varepsilon)^{\mathbb{N}}: \operatorname{dist}\left(f_{\underline{\omega}}^{j}(\zeta), \xi_{i}\right)>2 \sigma^{j}\left|U_{m}\right|\right\} .
$$

Observe that in this case $W_{k, m}^{c} \subset \bigcup_{j=1}^{k+1} \bigcup_{i=0}^{\ell}\left\{\underline{\omega}: f_{\underline{\omega}}^{j}(\zeta) \in B_{2 \sigma^{j}\left|U_{m}\right|}\left(\xi_{i}\right)\right\}$. Hence, we have

$$
\begin{aligned}
\theta_{\varepsilon}^{\mathbb{N}}\left(W_{k, m}^{c}\right) & \leq \sum_{i=0}^{\ell} \sum_{j=1}^{k+1} \int \theta_{\varepsilon}\left(\left\{\omega_{j}: f\left(f_{\underline{\omega}}^{j-1}(\zeta)\right)+\omega_{j} \in B_{2 \sigma^{j}\left|U_{m}\right|}\left(\xi_{i}\right)\right\}\right) \mathrm{d} \theta_{\varepsilon}^{\mathbb{N}} \\
& \leq \sum_{i=0}^{\ell} \sum_{j=1}^{k+1} \overline{g_{\varepsilon}}\left|B_{2 \sigma^{j}\left|U_{m}\right|}\left(\xi_{i}\right)\right|=\sum_{i=0}^{\ell} \sum_{j=1}^{k+1} \overline{g_{\varepsilon}} 4 \sigma^{j}\left|U_{m}\right| \\
& \leq 4(\ell+1) \overline{g_{\varepsilon}}\left|U_{m}\right| \frac{\sigma}{\sigma-1} \sigma^{k+1} .
\end{aligned}
$$

Using this estimate we obtain:

$$
\begin{aligned}
G_{k, m} & =\int_{W_{k, m}} \int \psi_{k, U_{m}, \underline{\omega}}(x) h_{\varepsilon}(x) \mathrm{d} \operatorname{Leb} \mathrm{d} \theta_{\varepsilon}^{\mathbb{N}}+\int_{W_{k, m}^{c}} \int \psi_{k, U_{m}, \underline{\omega}}(x) h_{\varepsilon}(x) \mathrm{d} \operatorname{Leb} \mathrm{d} \theta_{\varepsilon}^{\mathbb{N}} \\
& =0+\int_{W_{k, m}^{c}} \int \psi_{k, U_{m}, \underline{\omega}}(x) h_{\varepsilon}(x) \mathrm{d} \operatorname{Leb} \mathrm{d} \theta_{\varepsilon}^{\mathbb{N}}
\end{aligned}
$$

and because $\psi_{k, U_{m}, \underline{\omega}}(x) \leq \mathbf{1}_{U_{m}}(x)$, we have:

$$
\begin{aligned}
& \leq \int_{W_{k, m}^{c}} \int \mathbf{1}_{U_{m}}(x) h_{\varepsilon}(x) \mathrm{dLebd} \theta_{\varepsilon}^{\mathbb{N}} \leq \mu_{\varepsilon}\left(U_{m}\right) \theta_{\varepsilon}^{\mathbb{N}}\left(W_{k, m}^{c}\right) \\
& \leq \mu_{\varepsilon}\left(U_{m}\right) 4(\ell+1) \overline{g_{\varepsilon}}\left|U_{m}\right| \frac{\sigma}{\sigma-1} \sigma^{k+1} .
\end{aligned}
$$


Since $q_{k, m}=\frac{G_{k, m}}{\mu_{\varepsilon}\left(U_{m}\right)}$, we get

$$
q_{k, m} \leq \frac{\mu_{\varepsilon}\left(U_{m}\right) 4(\ell+1) \overline{g_{\varepsilon}}\left|U_{m}\right| \frac{\sigma}{\sigma-1} \sigma^{k+1}}{\mu_{\varepsilon}\left(U_{m}\right)} \leq 4(\ell+1) \overline{g_{\varepsilon}}\left|U_{m}\right| \frac{\sigma}{\sigma-1} \sigma^{k+1} \underset{m \rightarrow \infty}{\longrightarrow} 0 .
$$

Remark 5.1. Let us note that $D^{\prime}\left(u_{m}\right)$ implies that all $q_{k}$ 's are well defined and equal to 0 . Assume that there exists $k \in \mathbb{N}$ and a subsequence $\left(m_{i}\right)_{i \in \mathbb{N}}$ such that $\lim _{j \rightarrow \infty} \frac{G_{k, m_{j}}}{\mu_{\varepsilon}\left(U_{m_{j}}\right)}=\alpha>0$. Let us prove that $D^{\prime}\left(u_{m}\right)$ does not hold in this situation. Recall that if $D^{\prime}\left(u_{m}\right)$ holds, then

$$
\lim _{m \rightarrow \infty} m \sum_{j=1}^{\left\lfloor m / k_{m}\right\rfloor} \mu_{\varepsilon} \times \theta_{\varepsilon}^{\mathbb{N}}\left(X_{0}>u_{m}, X_{j}>u_{m}\right)=0,
$$

where $k_{m}$ (which should not be confused with $k$, here) is a sequence diverging to $\infty$ but slower than $m$, which implies that $\left\lfloor m / k_{m}\right\rfloor \rightarrow \infty$, as $m \rightarrow \infty$. Hence, let $M_{0}$ be sufficiently large so that for all $m>M_{0}$ we have $\left\lfloor m / k_{m}\right\rfloor>k$. Hence, for $i$ sufficiently large so that $m_{i}>M_{0}$, we may write

$$
\begin{aligned}
& m_{i} \sum_{j=1}^{\left\lfloor m_{i} / k_{m_{i}}\right\rfloor} \mu_{\varepsilon} \times \theta_{\varepsilon}^{\mathbb{N}}\left(X_{0}>u_{m_{i}}, X_{j}>u_{m_{i}}\right) \\
& \geq m_{i} \mu_{\varepsilon} \times \theta_{\varepsilon}^{\mathbb{N}}\left(X_{0}>u_{m_{i}}, X_{k+1}>u_{m_{i}}\right) \\
& \quad \geq m_{i} G_{k, m_{i}} \sim \frac{\tau G_{k, m_{i}}}{\mu_{\varepsilon}\left(U_{m_{i}}\right)} \rightarrow \tau \alpha>0, \text { as } i \rightarrow \infty,
\end{aligned}
$$

since $B_{m}$ is such that $m \mu_{\varepsilon}\left(U_{m}\right) \rightarrow \tau$, as $m \rightarrow \infty$. This implies that $D^{\prime}\left(u_{m}\right)$ does not hold.

\section{Appendix A. Clustering And Periodicity}

Condition $D^{\prime}\left(u_{n}\right)$ prevents the existence of clusters of exceedances, which implies that the EVL is standard exponential $\bar{H}(\tau)=\mathrm{e}^{-\tau}$. However, when $D^{\prime}\left(u_{n}\right)$ fails, clustering of exceedances is responsible for the appearance of a parameter $0<\vartheta<1$ in the EVL, called the EI, which implies that, in this case, $\bar{H}(\tau)=\mathrm{e}^{-\vartheta \tau}$. In [12], the authors established a connection between the existence of an EI less than 1 and periodic behaviour. This was later generalised for REPP in [13. Namely, this phenomenon of clustering appeared when $\zeta$ was a repelling periodic point. We assume that the invariant measure $\mathbb{P}$ and the observable $\varphi$ are sufficiently regular so that besides (R1), we also have that

(R2) If $\zeta \in \mathcal{X}$ is a repelling periodic point, of prime period $p \in \mathbb{N}$, then we have that the periodicity of $\zeta$ implies that for all large $u,\left\{X_{0}>u\right\} \cap$ $f^{-p}\left(\left\{X_{0}>u\right\}\right) \neq \emptyset$, and the fact that the prime period is $p$ implies that $\left\{X_{0}>u\right\} \cap f^{-j}\left(\left\{X_{0}>u\right\}\right)=\emptyset$ for all $j=1, \ldots, p-1$. Moreover, the fact that $\zeta$ is repelling means that we have backward contraction which means that there exists $0<\vartheta<1$ so that $\bigcap_{j=0}^{i} f^{-j p}\left(X_{0}>u\right)$ corresponds to another ball of smaller radius around $\zeta$ with $\mathbb{P}\left(\bigcap_{j=0}^{i} f^{-j p}\left(X_{0}>u\right)\right) \sim$ $(1-\vartheta)^{i} \mathbb{P}\left(X_{0}>u\right)$, for all $u$ sufficiently close to $u_{F}$.

\footnotetext{
${ }^{6}$ That is, the smallest $n \in \mathbb{N}$ such that $f^{n}(\zeta)=\zeta$. Clearly $f^{i p}(\zeta)=\zeta$ for any $i \in \mathbb{N}$.
} 
The main obstacle when dealing with periodic points is that they create plenty of dependence in the short range. In particular, using (R2) we have that for all $u$ sufficiently large, $\mathbb{P}\left(\left\{X_{0}>u\right\} \cap\left\{X_{p}>u\right\}\right) \sim(1-\vartheta) \mathbb{P}\left(X_{0}>u\right)$, which implies that $D^{\prime}\left(u_{n}\right)$ is not satisfied, since for the levels $u_{n}$ as in (2.10) it follows that $n \sum_{j=1}^{\left[n / k_{n}\right]} \mathbb{P}\left(X_{0}>u_{n}, X_{j}>u_{n}\right) \geq n \mathbb{P}\left(X_{0}>u_{n}, X_{p}>u_{n}\right) \underset{n \rightarrow \infty}{\longrightarrow}(1-\vartheta) \tau$. To overcome this difficulty around periodic points we make a key observation that roughly speaking tells us that around periodic points one just needs to replace the topological ball $\left\{X_{0}>u_{n}\right\}$ by the topological annulus

$$
Q_{p}(u):=\left\{X_{0}>u, X_{p} \leq u\right\} .
$$

Then much of the analysis works out as in the absence of clustering. Note that $Q_{p}(u)$ is obtained by removing from $U(u)$ the points that were doomed to return after $p$ steps, which form the smaller ball $U(u) \cap f^{-p}(U(u))$. Then, the crucial observation is that the limit law corresponding to no entrances up to time $n$ into the ball $U\left(u_{n}\right)$ is equal to the limit law corresponding to no entrances into the annulus $Q_{p}\left(u_{n}\right)$ up to time $n$.

In what follows, for every $A \in \mathcal{B}$ we denote the complement of $A$ as $A^{c}:=\mathcal{X} \backslash A$. For $s \leq \ell \in \mathbb{N}_{0}$, we define

$$
\mathscr{Q}_{p, s, \ell}(u)=\bigcap_{i=s}^{s+\ell-1} f^{-i}\left(Q_{p}(u)\right)^{c},
$$

which corresponds to no entrances in the annulus from time $s$ to $s+\ell-1$. Sometimes to abbreviate we also write $\mathscr{Q}_{\ell}(u):=\mathscr{Q}_{p, 0, \ell}(u)$.

Theorem G ([12, Proposition 1]). Let $X_{0}, X_{1}, \ldots$ be a stochastic process defined by (2.6) where $\varphi$ achieves a global maximum at a repelling periodic point $\zeta \in \mathcal{X}$, of prime period $p \in \mathbb{N}$, so that conditions (R1) and (R2) above hold. Let $\left(u_{n}\right)_{n}$ be a sequence of levels such that (2.10) holds. Then, $\lim _{n \rightarrow \infty} \mathbb{P}\left(M_{n} \leq u_{n}\right)=$ $\lim _{n \rightarrow \infty} \mathbb{P}\left(\mathscr{Q}_{n}\left(u_{n}\right)\right)$.

Hence, the idea to cope with clustering caused by periodic points is to adapt conditions $D_{2}\left(u_{n}\right)$ and $D^{\prime}\left(u_{n}\right)$, letting annuli replace balls.

Condition $\left(D^{p}\left(u_{n}\right)\right)$. We say that $D^{p}\left(u_{n}\right)$ holds for the sequence $X_{0}, X_{1}, X_{2}, \ldots$ if for any integers $\ell, t$ and $n,\left|\mathbb{P}\left(Q_{p, 0}\left(u_{n}\right) \cap \mathscr{Q}_{p, t, \ell}\left(u_{n}\right)\right)-\mathbb{P}\left(Q_{p, 0}\left(u_{n}\right)\right) \mathbb{P}\left(\mathscr{Q}_{p, 0, \ell}\left(u_{n}\right)\right)\right| \leq$ $\gamma(n, t)$, where $\gamma(n, t)$ is non-increasing in $t$ for each $n$ and $n \gamma\left(n, t_{n}\right) \rightarrow 0$ as $n \rightarrow \infty$ for some sequence $t_{n}=o(n)$.

As with $D_{2}\left(u_{n}\right)$, the main advantage of this condition when compared to Leadbetter's $D\left(u_{n}\right)$ (or others of the same sort) is that it follows directly from sufficiently fast decay of correlations as observed in [14, Section 5.1], contrary to $D\left(u_{n}\right)$.

Assuming $D^{p}\left(u_{n}\right)$ holds, let $\left(k_{n}\right)_{n \in \mathbb{N}}$ be a sequence of integers such that (2.13) holds.

Condition $\left(D_{p}^{\prime}\left(u_{n}\right)\right)$. We say that $D_{p}^{\prime}\left(u_{n}\right)$ holds for $X_{0}, X_{1}, X_{2}, \ldots$ if there exists a sequence $\left(k_{n}\right)_{n \in \mathbb{N}}$ satisfying (2.13) and such that

$$
\lim _{n \rightarrow \infty} n \sum_{j=1}^{\left[n / k_{n}\right]} \mathbb{P}\left(Q_{p, 0}\left(u_{n}\right) \cap Q_{p, j}\left(u_{n}\right)\right)=0 .
$$


One of the main results in [12] is:

Theorem H $\left(\left[12\right.\right.$, Theorem 1]). Let $\left(u_{n}\right)_{n \in \mathbb{N}}$ be such that (2.10) holds. Consider a stationary stochastic process $X_{0}, X_{1}, \ldots$ to be a stochastic process defined by (2.6) where $\varphi$ achieves a global maximum at a repelling periodic point $\zeta \in \mathcal{X}$, of prime period $p \in \mathbb{N}$, so that conditions (R1) and (R2) above hold. Assume further that conditions $D^{p}\left(u_{n}\right)$ and $D_{p}^{\prime}\left(u_{n}\right)$ hold. Then $\lim _{n \rightarrow \infty} \mathbb{P}\left(M_{n} \leq u_{n}\right)=$ $\lim _{n \rightarrow \infty} \mathbb{P}\left(\mathscr{Q}_{p, 0, n}\left(u_{n}\right)\right)=\mathrm{e}^{-\vartheta \tau}$.

Regarding the convergence of the REPP, when there is clustering, one cannot use the aforementioned criterion of Kallenberg because the point processes are not simple anymore and possess multiple events. This means that a much deeper analysis must be done in order to obtain convergence of the REPP. We carried this out in 13 and describe below the main results and conditions needed. First, we define the sequence $\left(U^{(\kappa)}(u)\right)_{\kappa \geq 0}$ of nested balls centred at $\zeta$ given by

$$
U^{(0)}(u)=U(u) \quad \text { and } \quad U^{(\kappa)}(u)=f^{-p}\left(U^{(\kappa-1)}(u)\right) \cap U(u), \quad \text { for all } \kappa \in \mathbb{N} .
$$

For $i, \kappa, \ell, s \in \mathbb{N} \cup\{0\}$, we define the following events:

$$
Q_{p, i}^{\kappa}(u):=f^{-i}\left(U^{(\kappa)}(u)-U^{(\kappa+1)}(u)\right) .
$$

Observe that for each $\kappa$, the set $Q_{p, 0}^{\kappa}(u)$ corresponds to an annulus centred at $\zeta$. Besides, $U(u)=\bigcup_{\kappa=0}^{\infty} Q_{p, 0}^{\kappa}(u)$, which means that the ball centred at $\zeta$ which corresponds to $U(u)$ can be decomposed into a sequence of disjoint annuli where $Q_{p, 0}^{0}(u)$ is the most outward ring and the inner $\operatorname{ring} Q_{p, 0}^{\kappa+1}(u)$ is sent outward by $f^{p}$ to the ring $Q_{p, 0}^{\kappa}(u)$, i.e., $f^{p}\left(Q_{p, 0}^{\kappa+1}(u)\right)=Q_{p, 0}^{\kappa}(u)$.

We are now ready to state:

Condition $\left(D_{p}\left(u_{n}\right)^{*}\right)$. We say that $D_{p}\left(u_{n}\right)^{*}$ holds for the sequence $X_{0}, X_{1}, X_{2}, \ldots$ if for any integers $t, \kappa_{1}, \ldots, \kappa_{\varsigma}, n$ and any $J=\bigcup_{i=2}^{\varsigma} I_{j} \in \mathcal{R}$ with $\inf \{x: x \in J\} \geq t$,

$$
\left|\mathbb{P}\left(Q_{p, 0}^{\kappa_{1}}\left(u_{n}\right) \cap\left(\bigcap_{j=2}^{\varsigma} \mathscr{N}_{u_{n}}\left(I_{j}\right)=\kappa_{j}\right)\right)-\mathbb{P}\left(Q_{p, 0}^{\kappa_{1}}\left(u_{n}\right)\right) \mathbb{P}\left(\bigcap_{j=2}^{\varsigma} \mathscr{N}_{u_{n}}\left(I_{j}\right)=\kappa_{j}\right)\right|
$$

where for each $n$ we have that $\gamma(n, t)$ is non-increasing in $t$ and $n \gamma\left(n, t_{n}\right) \rightarrow 0$ as $n \rightarrow \infty$, for some sequence $t_{n}=o(n)$.

This mixing condition is stronger than $D^{p}\left(u_{n}\right)$ because it requires a uniform bound for all possible integer values of $\kappa_{1}$; nonetheless, it still is much weaker than the original $D\left(u_{n}\right)$ from Leadbetter 25] or any of this kind. As with all the other preceding conditions $\left(D_{2}, D_{3}, D^{p}\right)$, it can be easily verified for systems with sufficiently fast decay of correlations (see [14, Section 5.1]).

In 13, for technical reasons only, we also introduced a slight modification to $D_{p}^{\prime}\left(u_{n}\right)$. The new condition was denoted by $D_{p}^{\prime}\left(u_{n}\right)^{*}$ and the difference is that we require that $\lim _{n \rightarrow \infty} n \sum_{j=1}^{\left[n / k_{n}\right]} \mathbb{P}\left(Q_{p, 0}\left(u_{n}\right) \cap\left\{X_{j}>u_{n}\right\}\right)=0$ holds.

We can now state the main theorem in [13].

Theorem I ([13, Theorem 1]). Let $X_{0}, X_{1}, \ldots$ be given by (2.6), where $\varphi$ achieves a global maximum at the repelling periodic point $\zeta$, of prime period $p$, and conditions (R1) and (R2) hold. Let $\left(u_{n}\right)_{n \in \mathbb{N}}$ be a sequence satisfying (2.10). Assume that 
conditions $D_{p}\left(u_{n}\right)^{*}, D_{p}^{\prime}\left(u_{n}\right)^{*}$ hold. Then the REPP $N_{n}$ converges in distribution to a compound Poisson process $N$ with intensity $\vartheta$ and multiplicity d.f. $\pi$ given by $\pi(\kappa)=\vartheta(1-\vartheta)^{\kappa}$, for every $\kappa \in \mathbb{N}_{0}$, where the extremal index $\vartheta$ is given by the expansion rate at $\zeta$ stated in $(\mathrm{R} 2)$.

\section{Appendix B. Computing the EI And the Multiplicity Distribution}

In order to prove the existence of an EI around a repelling periodic point, we may use Theorem $\mathrm{H}$ and, basically, observe that, once conditions $D^{p}\left(u_{n}\right)$ and $D_{p}^{\prime}\left(u_{n}\right)$ are verified, by (R2) the EI may be computed from the formula

$$
\vartheta=\lim _{n \rightarrow \infty} \frac{\mathbb{P}\left(Q_{p, 0}\left(u_{n}\right)\right)}{\mathbb{P}\left(U_{n}\right)} .
$$

In order to compute the multiplicity distribution of the limiting compound Poisson process for the REPP, when $\zeta$ is a repelling periodic point, we can use the following estimate:

Lemma B.1 ([13, Corollary 2.4]). Assuming that $\varphi$ achieves a global maximum at the repelling periodic point $\zeta$, of prime period $p$, and conditions (R1) and (R2) hold, there exists $C>0$ depending only on $\vartheta$ given by property (R2) such that for any $s, \kappa \in \mathbb{N}$ and $u$ sufficiently close to $u_{F}=\varphi(\zeta)$ we have for $\kappa>0$

$$
\begin{aligned}
& \left|\mathbb{P}\left(\mathscr{N}_{u, 0}^{s+1}=\kappa\right)-s\left(\mathbb{P}\left(Q_{p, 0}^{\kappa-1}(u)\right)-\mathbb{P}\left(Q_{p, 0}^{\kappa}(u)\right)\right)\right| \\
& \quad \leq 4 s \sum_{j=p+1}^{s} \mathbb{P}\left(Q_{p, 0}^{0}(u) \cap\left\{X_{j}>u\right\}\right)+2 C \mathbb{P}\left(X_{0}>u_{n}\right),
\end{aligned}
$$

and in the case $\kappa=0$

$$
\begin{aligned}
& \left|\mathbb{P}\left(\mathscr{N}_{u, 0}^{s+1}=0\right)-\left(1-s \mathbb{P}\left(Q_{p, 0}^{0}(u)\right)\right)\right| \\
& \quad \leq 2 s \sum_{j=p+1}^{s} \mathbb{P}\left(Q_{p, 0}^{0}(u) \cap\left\{X_{j}>u\right\}\right)+C \mathbb{P}\left(X_{0}>u\right) .
\end{aligned}
$$

The idea then is to realise that in the proof of Theorem $\square$ one splits the first $n$ r.v. $X_{0}, \ldots, X_{n-1}$ into blocks of size $\left\lfloor n / k_{n}\right\rfloor$ with a time gap of size $t_{n}$ between them. Then using the asymptotic "independence" obtained from $D^{p}\left(u_{n}\right)^{*}$ and $D_{p}^{\prime}\left(u_{n}\right)^{*}$ we get the compound Poisson limit with multiplicity distribution determined by the distributional limit of the number of exceedances in each block of size $\left\lfloor n / k_{n}\right\rfloor$, given that at least one exceedance occurs. Hence, we need to compute, for all $\kappa \in \mathbb{N}, \lim _{n \rightarrow \infty} \mathbb{P}\left(\mathscr{N}_{u_{n}, 0}^{\left\lfloor n / k_{n}\right\rfloor+1}=\kappa \mid \mathscr{N}_{u_{n}, 0}^{\left\lfloor n / k_{n}\right\rfloor+1}>0\right)$. Since, by $D_{p}^{\prime}\left(u_{n}\right)^{*}$, we have that $\left\lfloor n / k_{n}\right\rfloor \sum_{j=p+1}^{\left\lfloor n / k_{n}\right\rfloor} \mathbb{P}\left(Q_{p, 0}^{0}\left(u_{n}\right) \cap\left\{X_{j}>u_{n}\right\}\right)=o\left(1 / k_{n}\right)$, then by Lemma B.1 we have that, for every $\kappa \in \mathbb{N}$,

$$
\begin{aligned}
\pi(\kappa) & =\lim _{n \rightarrow \infty} \mathbb{P}\left(\mathscr{N}_{u_{n}, 0}^{\left\lfloor n / k_{n}\right\rfloor+1}=\kappa \mid \mathscr{N}_{u_{n}, 0}^{\left\lfloor n / k_{n}\right\rfloor+1}>0\right) \\
& =\lim _{n \rightarrow \infty} \frac{\left(\mathbb{P}\left(Q_{p, 0}^{\kappa-1}\left(u_{n}\right)\right)-\mathbb{P}\left(Q_{p, 0}^{\kappa}\left(u_{n}\right)\right)\right)}{\mathbb{P}\left(Q_{p, 0}^{0}\left(u_{n}\right)\right)} .
\end{aligned}
$$




\section{ACKNOWLEDGEMENTS}

We wish to thank Ian Melbourne for helpful comments and, in particular, for calling our attention to the paper [7] in this context. We are grateful to Wael Bahsoun and Manuel Stadlbauer for fruitful conversations and comments. We are also grateful to Mike Todd for a careful reading and many suggestions that improved the paper.

\section{REFERENCES}

[1] José F. Alves, Jorge M. Freitas, Stefano Luzzatto, and Sandro Vaienti, From rates of mixing to recurrence times via large deviations, Adv. Math. 228 (2011), no. 2, 1203-1236, DOI 10.1016/j.aim.2011.06.014. MR2822221 (2012g:37049)

[2] José Ferreira Alves and Vítor Araújo, Random perturbations of nonuniformly expanding maps (English, with English and French summaries), Astérisque 286 (2003), xvii, 25-62. Geometric methods in dynamics. I. MR2052296 (2005e:37058)

[3] Viviane Baladi, Michael Benedicks, and Véronique Maume-Deschamps, Almost sure rates of mixing for i.i.d. unimodal maps (English, with English and French summaries), Ann. Sci. École Norm. Sup. (4) 35 (2002), no. 1, 77-126, DOI 10.1016/S0012-9593(01)01083-7. MR.1886006 (2003d:37027)

[4] Viviane Baladi, Michael Benedicks, and Véronique Maume-Deschamps, Corrigendum: "Almost sure rates of mixing for i.i.d. unimodal maps" [Ann. Sci. École Norm. Sup. (4) 35 (2002), no. 1, 77-126; MR1886006 (2003d:37027)], Ann. Sci. École Norm. Sup. (4) 36 (2003), no. 2, 319-322. MR1980314 (2004e:37050)

[5] Abraham Boyarsky and PaweLeb Góra, Laws of chaos, Probability and its Applications, Birkhäuser Boston Inc., Boston, MA, 1997. Invariant measures and dynamical systems in one dimension. MR 1461536(99a:58102)

[6] Jean-René Chazottes, Zaqueu Coelho, and Pierre Collet, Poisson processes for subsystems of finite type in symbolic dynamics, Stoch. Dyn. 9 (2009), no. 3, 393-422, DOI 10.1142/S0219493709002713. MR2566908 (2011g:37014)

[7] Dmitry Dolgopyat, On decay of correlations in Anosov flows, Ann. of Math. (2) 147 (1998), no. 2, 357-390, DOI 10.2307/121012. MR1626749 (99g:58073)

[8] Andrew Ferguson and Mark Pollicott, Escape rates for Gibbs measures, Ergodic Theory Dynam. Systems 32 (2012), no. 3, 961-988, DOI 10.1017/S0143385711000058. MR2995652

[9] Ana Cristina Moreira Freitas and Jorge Milhazes Freitas, On the link between dependence and independence in extreme value theory for dynamical systems, Statist. Probab. Lett. $\mathbf{7 8}$ (2008), no. 9, 1088-1093, DOI 10.1016/j.spl.2007.11.002. MR 2422964(2009e:37006)

[10] Ana Cristina Moreira Freitas, Jorge Milhazes Freitas, and Mike Todd, Hitting time statistics and extreme value theory, Probab. Theory Related Fields 147 (2010), no. 3-4, 675-710, DOI 10.1007/s00440-009-0221-y. MR2639719 (2011g:37015)

[11] Ana Cristina Moreira Freitas, Jorge Milhazes Freitas, and Mike Todd, Extreme value laws in dynamical systems for non-smooth observations, J. Stat. Phys. 142 (2011), no. 1, 108-126, DOI 10.1007/s10955-010-0096-4. MR2749711 (2012a:60149)

[12] Ana Cristina Moreira Freitas, Jorge Milhazes Freitas, and Mike Todd, The extremal index, hitting time statistics and periodicity, Adv. Math. 231 (2012), no. 5, 2626-2665, DOI 10.1016/j.aim.2012.07.029. MR.2970462

[13] Ana Cristina Moreira Freitas, Jorge Milhazes Freitas, and Mike Todd, The compound Poisson limit ruling periodic extreme behaviour of non-uniformly hyperbolic dynamics, Comm. Math. Phys. 321 (2013), no. 2, 483-527, DOI 10.1007/s00220-013-1695-0. MR.3063917

[14] Jorge Milhazes Freitas, Extremal behaviour of chaotic dynamics, Dyn. Syst. 28 (2013), no. 3, 302-332, DOI 10.1080/14689367.2013.806731. MR3170619

[15] N. Haydn, Y. Lacroix, and S. Vaienti, Hitting and return times in ergodic dynamical systems, Ann. Probab. 33 (2005), no. 5, 2043-2050, DOI 10.1214/009117905000000242. MR2165587 (2006i:37006)

[16] Nicolai Haydn and Sandro Vaienti, The compound Poisson distribution and return times in dynamical systems, Probab. Theory Related Fields 144 (2009), no. 3-4, 517-542, DOI 10.1007/s00440-008-0153-y. MR2496441 (2010i:37015) 
[17] Masaki Hirata, Poisson law for Axiom A diffeomorphisms, Ergodic Theory Dynam. Systems 13 (1993), no. 3, 533-556, DOI 10.1017/S0143385700007513. MR.1245828 (94m:58137)

[18] Olav Kallenberg, Random measures, 4th ed., Akademie-Verlag, Berlin, $1986 . \quad$ MR.854102 (87k:60137)

[19] Gerhard Keller, Stochastic stability in some chaotic dynamical systems, Monatsh. Math. 94 (1982), no. 4, 313-333, DOI 10.1007/BF01667385. MR685377 (84k:58130)

[20] Gerhard Keller, Rare events, exponential hitting times and extremal indices via spectral perturbation, Dyn. Syst. 27 (2012), no. 1, 11-27, DOI 10.1080/14689367.2011.653329. MR2903242

[21] Gerhard Keller and Carlangelo Liverani, Rare events, escape rates and quasistationarity: some exact formulae, J. Stat. Phys. 135 (2009), no. 3, 519-534, DOI 10.1007/s10955-0099747-8. MR2535206 (2011a:37012)

[22] Yuri Kifer, Ergodic theory of random transformations, Progress in Probability and Statistics, vol. 10, Birkhäuser Boston Inc., Boston, MA, 1986. MR884892 (89c:58069)

[23] Yuri Kifer and Pei-Dong Liu, Random dynamics, Handbook of dynamical systems. Vol. 1B, Elsevier B. V., Amsterdam, 2006, pp. 379-499, DOI 10.1016/S1874-575X(06)80030-5. MR2186245(2008a:37002)

[24] Yuri Kifer and Ariel Rapaport, Poisson and compound Poisson approximations in a nonconventional setup, Probability and Related Fields, DOI 10.1007/s00440-013-0541-9.

[25] M. R. Leadbetter, On extreme values in stationary sequences, Z. Wahrscheinlichkeitstheorie und Verw. Gebiete 28 (1973/74), 289-303. MR0362465 (50 \#14906)

[26] Philippe Marie and Jérôme Rousseau, Recurrence for random dynamical systems, Discrete Contin. Dyn. Syst. 30 (2011), no. 1, 1-16, DOI 10.3934/dcds.2011.30.1. MR2773129 (2012c:37108)

[27] Marek Rychlik, Bounded variation and invariant measures, Studia Math. 76 (1983), no. 1, 69-80. MR728198(85h:28019)

[28] Benoît Saussol, Absolutely continuous invariant measures for multidimensional expanding maps, Israel J. Math. 116 (2000), 223-248, DOI 10.1007/BF02773219. MR1759406 (2001e:37037)

[29] Benoit Saussol, An introduction to quantitative Poincaré recurrence in dynamical systems, Rev. Math. Phys. 21 (2009), no. 8, 949-979, DOI 10.1142/S0129055X09003785. MR2568049 (2011c:37031)

[30] Marcelo Viana, Stochastic dynamics of deterministic systems, Brazillian Math. Colloquium 1997, IMPA, 1997.

[31] Peter Walters, An introduction to ergodic theory, Graduate Texts in Mathematics, vol. 79, Springer-Verlag, New York, 1982. MR648108(84e:28017)

Centro de Matemática, Universidade do Porto, Rua do Campo Alegre 687, 4169-007 Porto, Portugal

Current address: Universidade Federal da Bahia, Instituto de Matemática, Av. Adhemar de

Barros, S/N, Ondina, 40170-110 Salvador-BA, Brazil

E-mail address: aytach@fc.up.pt

Centro de Matemática \& Faculdade de Ciências, Universidade do Porto, Rua do Campo Alegre 687, 4169-007 Porto, Portugal

E-mail address: jmfreita@fc.up.pt

$U R L:$ http://www.fc.up.pt/pessoas/jmfreita

Aix Marseille Université, CNRS, CPT, UMR 7332, 13288 Marseille, France - And Université de Toulon, CNRS, CPT, UMR 7332, 83957 La Garde, France

E-mail address: vaienti@cpt.univ-mrs.fr 